\title{
Complete scalar-flat Kähler metrics on affine algebraic manifolds
}

\section{Takahiro Aoi ${ }^{1}$}

Received: 21 February 2020 / Accepted: 4 December 2020 / Published online: 26 January 2021

(c) The Author(s) 2021

\begin{abstract}
Let $\left(X, L_{X}\right)$ be an $n$-dimensional polarized manifold. Let $D$ be a smooth hypersurface defined by a holomorphic section of $L_{X}$. We prove that if $D$ has a constant positive scalar curvature Kähler metric, $X \backslash D$ admits a complete scalar-flat Kähler metric, under the following three conditions: (i) $n \geq 6$ and there is no nonzero holomorphic vector field on $X$ vanishing on $D$, (ii) the average of a scalar curvature on $D$ denoted by $\hat{S}_{D}$ satisfies the inequality $0<3 \hat{S}_{D}<n(n-1)$, (iii) there are positive integers $l(>n), m$ such that the line bundle $K_{X}^{-l} \otimes L_{X}^{m}$ is very ample and the ratio $m / l$ is sufficiently small.
\end{abstract}

Keywords Constant scalar curvature Kähler metrics · Complex Monge-Ampère equations · Plurisubharmonic functions · Asymptotically conical geometry · Fredholm operators ·

Kähler manifolds

Mathematics Subject Classification 53C25 · 32Q15 · 53C21

\section{Contents}

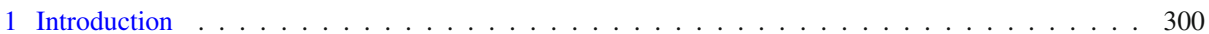

2 The case of line bundles . . . . . . . . . . . . . . . . . . . . . . . . . . . . 304

2.1 LeBrun-Simanca metrics . . . . . . . . . . . . . . . . . . . . . . 305

2.2 The extension to the total space . . . . . . . . . . . . . . . . . . . 306

2.3 The Ricci form and the scalar curvature . . . . . . . . . . . . . . . . . . . . . . 307

$2.4 \mathrm{ODE} \ldots \ldots \ldots \ldots \ldots \ldots \ldots \ldots$

2.5 Volume growth . . . . . . . . . . . . . . . . . . . . . . . . . 308

3 The higher order decay . . . . . . . . . . . . . . . . . . . . . . . 311

3.1 Matrix analysis . . . . . . . . . . . . . . . . . . . . . . . 312

3.2 Local trivialization and normal coordinates . . . . . . . . . . . . . . . . . . 313

3.3 Proof of Theorem $1 \ldots \ldots \ldots \ldots \ldots$

4 Asymptotically conical geometry . . . . . . . . . . . . . . . . . . . . . . . . . . . . . . . 320

5 Forth order elliptic linear operators . . . . . . . . . . . . . . . . . . . . . . . 321

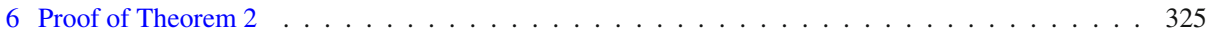

7 Plurisubharmonic functions with small scalar curvature . . . . . . . . . . . . . . . . . . 327

Takahiro Aoi

t-aoi@cr.math.sci.osaka-u.ac.jp

1 Department of Mathematics, Graduate School of Science, Osaka University, Toyonaka 560-0043, Japan 
7.1 Kähler potential near $D \ldots \ldots \ldots \ldots \ldots \ldots \ldots$. . . . . . . . . . . . . . 327

7.2 Kähler potential near $F \ldots \ldots \ldots \ldots$. . . . . . . . . . . . . . . . . 328

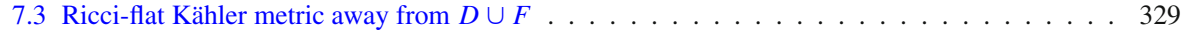

7.4 Gluing plurisubharmonic functions . . . . . . . . . . . . . . . . . . . . . 330

8 Proof of Theorem $3 \ldots \ldots \ldots \ldots \ldots$

8.1 The $C^{2}$-estimate . . . . . . . . . . . . . . . . . . . . . . . 332

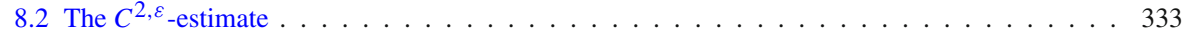

8.3 The third and the forth order estimates . . . . . . . . . . . . . . . . . . . 338

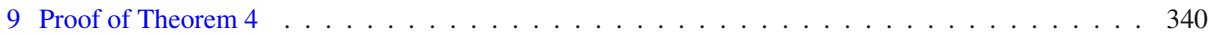

10 Proof of Theorem $5 \ldots \ldots \ldots \ldots \ldots \ldots$

11 Proof of Theorem $6 \ldots \ldots \ldots \ldots \ldots \ldots \ldots$

11.1Condition 1 and Condition $2 \ldots \ldots \ldots \ldots \ldots \ldots \ldots \ldots$

11.2The fixed point theorem . . . . . . . . . . . . . . . . . . . 353

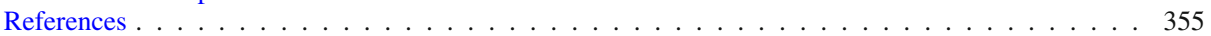

\section{Introduction}

The existence of constant scalar curvature Kähler ( $\operatorname{cscK})$ metrics on complex manifolds is a fundamental problem in Kähler geometry. If a complex manifold is noncompact, there are many positive results in this problem. In 1979, Calabi [5] showed that if a Fano manifold has a Kähler Einstein metric, then there is a complete Ricci-flat Kähler metric on the total space of the canonical line bundle. In addition, there exist following generalizations. In 1990, BandoKobayashi [3] showed that if a Fano manifold admits an anti-canonical smooth divisor which has a Ricci-positive Kähler Einstein metric, then there exists a complete Ricci-flat Kähler metric on the complement (see also [19]). Tian-Yau [18] showed that if a Fano manifold admits an anti-canonical smooth divisor which has a Ricci-flat Kähler metric, then there is a complete Ricci-flat Kähler metric on the complement. In 2002, on the other hand, as a scalar curvature version of Calabi's result [5], Hwang-Singer [12] showed that if a polarized manifold has a nonnegative cscK metric, then the total space of the dual line bundle admits a complete scalar-flat Kähler metric. However, a similar generalization of Hwang-Singer [12] like Bando-Kobayashi [3] and Tian-Yau [18] is unknown since it is hard to solve a forth order nonlinear partial differential equation.

In this article, assuming the existence of a smooth hypersurface which admits a constant positive scalar curvature Kähler metric, we will prove the existence of a complete scalar-flat Kähler metric on the complement of this hypersurface. Our proof goes roughly as follows. Step 1 . We show that if the smooth hypersurface has a cscK metric, there is a complete Kähler metric whose scalar curvature decays at a higher order.

Step 2. We show that the existence of a complete Kähler metric whose scalar curvature is sufficiently small implies the existence of a complete scalar-flat Kähler metric.

Step 3. We construct a complete Kähler metric on the complement of the smooth hypersurface, whose scalar curvature is arbitrarily small.

Step 4. Finally, we show the existence of a complete scalar-flat Kähler metric by solving the forth order nonlinear partial differential equation.

Now we describe our strategy more precisely. Let $\left(X, L_{X}\right)$ be a polarized manifold of dimension $n$, i.e., $X$ is an $n$-dimensional compact complex manifold and $L_{X}$ is an ample line bundle over $X$. Assume that there is a smooth hypersurface $D \subset X$ with

$$
D \in\left|L_{X}\right| \text {. }
$$

Set an ample line bundle $L_{D}:=\left.\mathscr{O}(D)\right|_{D}=\left.L_{X}\right|_{D}$ over $D$. Since $L_{X}$ is ample, there exists a Hermitian metric $h_{X}$ on $L_{X}$ which defines a Kähler metric $\theta_{X}$ on $X$, i.e., the curvature form 
of $h_{X}$ multiplied by $\sqrt{-1}$ is positive definite. Then, the restriction of $h_{X}$ to $L_{D}$ defines also a Kähler metric $\theta_{D}$ on $D$. Let $\hat{S}_{D}$ be the average of the scalar curvature $S\left(\theta_{D}\right)$ of $\theta_{D}$ defined by

$$
\hat{S}_{D}:=\frac{\int_{D} S\left(\theta_{D}\right) \theta_{D}^{n-1}}{\int_{D} \theta_{D}^{n-1}}=\frac{(n-1) c_{1}\left(K_{D}^{-1}\right) \cup c_{1}\left(L_{D}\right)^{n-2}}{c_{1}\left(L_{D}\right)^{n-1}},
$$

where $K_{D}^{-1}$ is the anti-canonical line bundle of $D$. Note that $\hat{S}_{D}$ is a topological invariant in the sense that it is representable in terms of Chern classes of the line bundles $K_{D}^{-1}$ and $L_{D}$. In this article, we treat the following case:

$$
\hat{S}_{D}>0 .
$$

Let $\sigma_{D} \in H^{0}\left(X, L_{X}\right)$ be a defining section of $D$ and set $t:=\log \left\|\sigma_{D}\right\|_{h_{X}}^{-2}$. Following [3], we can define a complete Kähler metric $\omega_{0}$ by

$$
\omega_{0}:=\frac{n(n-1)}{\hat{S}_{D}} \sqrt{-1} \partial \bar{\partial} \exp \left(\frac{\hat{S}_{D}}{n(n-1)} t\right)
$$

on the noncompact complex manifold $X \backslash D$. In addition, since $\left(X \backslash D, \omega_{0}\right)$ is of asymptotically conical geometry (see [3] or Sect. 4 of this article), we can define the weighted Banach space $C_{\delta}^{k, \alpha}=C_{\delta}^{k, \alpha}(X \backslash D)$ for $k \in \mathbb{Z}_{\geq 0}, \alpha \in(0,1)$ and with a weight $\delta \in \mathbb{R}$ with respect to the distance function $r$ defined by $\omega_{0}$ from some fixed point in $X \backslash D$. It follows from the construction of $\omega_{0}$ that $S\left(\omega_{0}\right)=O\left(r^{-2}\right)$ near $D$.

Step 1 . The cscK condition implies the following stronger decay property.

Theorem 1 If $\theta_{D}$ is a constant positive scalar curvature Kähler metric on D, i.e., $S\left(\theta_{D}\right)=$ $\hat{S}_{D}>0$, we have

$$
S\left(\omega_{0}\right)=O\left(r^{-2-2 n(n-1) / \hat{S}_{D}}\right)
$$

as $r \rightarrow \infty$.

Thus, the $\operatorname{cscK}$ condition implies that $S\left(\omega_{0}\right) \in C_{\delta}^{k, \alpha}$ for some $\delta>2$ and any $k, \alpha$.

Step 2. In order to construct a complete scalar-flat Kähler metric on $X \backslash D$, the linearization of the scalar curvature operator plays an important role:

$$
L_{\omega_{0}}=-\mathscr{D}_{\omega_{0}}^{*} \mathscr{D}_{\omega_{0}}+\left(\nabla^{1,0} *, \nabla^{0,1} S\left(\omega_{0}\right)\right)_{\omega_{0}} .
$$

Here, $\mathscr{D}_{\omega_{0}}=\bar{\partial} \circ \nabla^{1,0}$. We will show that if $4<\delta<2 n$ and there is no nonzero holomorphic vector field on $X$ which vanishes on $D$, then $\mathscr{D}_{\omega_{0}}^{*} \mathscr{D}_{\omega_{0}}: C_{\delta-4}^{4, \alpha} \rightarrow C_{\delta}^{0, \alpha}$ is isomorphic. For such operators, we consider the following:

Condition 1 Assume that $n \geq 3$ and there is no nonzero holomorphic vector field on $X$ which vanishes on $D$. For $4<\delta<2 n$, the operator

$$
L_{\omega_{0}}: C_{\delta-4}^{4, \alpha} \rightarrow C_{\delta}^{0, \alpha}
$$

is isomorphic, i.e., we can find a constant $\hat{K}>0$ such that

$$
\left\|L_{\omega_{0}} \phi\right\|_{C_{\delta}^{0, \alpha}} \geq \hat{K}\|\phi\|_{C_{\delta-4}^{4, \alpha}}
$$

for any $\phi \in C_{\delta-4}^{4, \alpha}$. 
In addition, we consider

\section{Condition 2}

$$
\left\|S\left(\omega_{0}\right)\right\|_{C_{\delta}^{0, \alpha}}<c_{0} \hat{K} / 2 .
$$

Here, the constant $c_{0}$ will be defined in Lemma 15 later. Under these conditions, Theorem 1 implies the following result:

Theorem 2 Assume that $n \geq 3$ and there is no nonzero holomorphic vector field on $X$ which vanishes on D. Assume that $\theta_{D}$ is a constant scalar curvature Kähler metric satisfying

$$
0<\hat{S}_{D}<n(n-1) .
$$

Assume moreover that Condition 1 and Condition 2 hold, then $X \backslash D$ admits a complete scalar-flat Kähler metric.

In fact, we can show the existence of a complete scalar-flat Kähler metric on $X \backslash D$ under the following assumptions: (i) $n \geq 3$ and there is no nonzero holomorphic vector field on $X$ which vanishes on $D$, (ii) there exists a complete Kähler metric on $X \backslash D$ which is of asymptotically conical geometry, such that its scalar curvature is sufficiently small and decays at a higher order. So, if there exists a complete Kähler metric on $X \backslash D$ which is sufficiently close to $\omega_{0}$ at infinity, satisfying Conditions 1 and 2, we can show the existence of a complete scalar-flat Kähler metric on $X \backslash D$. Theorem 2 is proved by the fixed point theorem on the weighted Banach space $C_{\delta-4}^{4, \alpha}(X \backslash D)$ by following Arezzo-Pacard [1], [2] (see also [17]). In general, constants $c_{0}, \hat{K}$ which arise in Conditions 1 and 2 depend on the background Kähler metric $\omega_{0}$. In addition, to construct such a Kähler metric, we have to find a complete Kähler metric $X \backslash D$ whose scalar curvature is arbitrarily small.

Step 3. We consider a degenerate (meromorphic) complex Monge-Ampère equation. Take positive integers $l>n$ and $m$ such that the line bundle $K_{X}^{-l} \otimes L_{X}^{m}$ is very ample. Let $F \in\left|K_{X}^{-l} \otimes L_{X}^{m}\right|$ be a smooth hypersurface defined by a holomorphic section $\sigma_{F} \in$ $H^{0}\left(X, K_{X}^{-l} \otimes L_{X}^{m}\right)$ such that the divisor $D+F$ is simple normal crossing. For a defining section $\sigma_{D} \in H^{0}\left(X, L_{X}\right)$ of $D$, set

$$
\xi:=\sigma_{F} \otimes \sigma_{D}^{-m} .
$$

From the result due to Yau [20, Theorem 7], we can solve the following degenerate complex Monge-Ampère equation:

$$
\left(\theta_{X}+\sqrt{-1} \partial \bar{\partial} \varphi\right)^{n}=\xi^{-1 / l} \wedge \bar{\xi}^{-1 / l} .
$$

Moreover, it follows from a priori estimate due to Kołodziej [13] that the solution $\varphi$ is bounded on $X$. Thus, we can glue plurisubharmonic functions by using the regularized maximum function. To compute the scalar curvature of the glued Kähler metric, we need to study behaviors of higher order derivatives of the solution $\varphi$. So, we give explicit estimates of them near the intersection $D \cap F$ :

Theorem 3 Let $\left(z^{i}\right)_{i=1}^{n}=\left(z^{1}, z^{2}, \ldots, z^{n-2}, w_{F}, w_{D}\right)$ be local holomorphic coordinates such that $\left\{w_{F}=0\right\}=F$ and $\left\{w_{D}=0\right\}=D$. Then, there exists a positive integer $a(n)$ depending only on the dimension $n$ such that

$$
\left|\frac{\partial^{2}}{\partial z^{i} \partial \bar{z}^{j}} \partial^{\alpha} \varphi\right|=O\left(\left|w_{D}\right|^{-2 a(n) m / l}\left|w_{F}\right|^{-2 a(n) / l}\right),
$$




$$
\begin{aligned}
& \left|\frac{\partial^{4}}{\partial w_{F}^{2} \partial \overline{w_{F}^{2}}} \varphi\right|=O\left(\left|w_{D}\right|^{-2 a(n) m / l}\left|w_{F}\right|^{-2-2 a(n) / l}\right), \\
& \left|\frac{\partial^{4}}{\partial w_{D}^{2} \partial \overline{w_{D}^{2}}} \varphi\right|=O\left(\left|w_{D}\right|^{-2-2 a(n) m / l}\left|w_{F}\right|^{-2 a(n) / l}\right),
\end{aligned}
$$

as $\left|w_{F}\right|,\left|w_{D}\right| \rightarrow 0$, for any $1 \leq i, j \leq n-2$ and multi-index $\alpha=\left(\alpha_{1}, \ldots, \alpha_{n}\right)$ with $0 \leq \sum_{i} \alpha_{i} \leq 2$

By applying Theorem 3 and gluing plurisubharmonic functions, we have the following result:

Theorem 4 Assume that there exist positive integers $l>n$ and $m$ such that

$$
\frac{a(n) m}{2 l}<\frac{\hat{S}_{D}}{n(n-1)}
$$

and the line bundle $K_{X}^{-l} \otimes L_{X}^{m}$ is very ample. Here, $a(n)$ is the positive integer in Theorem 3. Take a smooth hypersurface $F \in\left|K_{X}^{-l} \otimes L_{X}^{m}\right|$ such that $D+F$ is simple normal crossing. Then, for any relatively compact domain $Y \Subset X \backslash(D \cup F)$, there exists a complete Kähler metric $\omega_{F}$ on $X \backslash D$ whose scalar curvature $S\left(\omega_{F}\right)=0$ on $Y$ and is arbitrarily small on the complement of $Y$. In addition, $\omega_{F}=\omega_{0}$ on some neighborhood of $D \backslash(D \cap F)$.

For example, if the anti-canonical line bundle $K_{X}^{-1}$ of the compact complex manifold $X$ is nef (in particular, $X$ is Fano), the assumption (2) in Theorem 4 holds, i.e., we can always find such integers $l, m$. In this article, we treat the case that $K_{X}^{-1}$ has positivity in the senses of (1) and (2). From Theorem 2, if there exists a complete Kähler metric which is of asymptotically conical geometry and satisfies Conditions 1 and 2, $X \backslash D$ admits a complete scalar-flat Kähler metric. In fact, Theorem 4 gives a Kähler metric whose scalar curvature is under control. However, the Kähler metric $\omega_{F}$ in Theorem 4 is not of asymptotically conical geometry (near the intersection of $D$ and $F$ ). So, when we replace the complete Kähler metric $\omega_{0}$ with $\omega_{F}$ obtained in Theorem 4, we can not apply Theorem 2 to a construction of a complete scalar-flat Kähler metric.

To solve this problem, we consider an average on some closed subset in $\left|K_{X}^{-l} \otimes L_{X}^{m+\beta}\right|$. Then, the asymptotically conicalness is recovered and we obtain the following result:

Theorem 5 Assume that there are positive integers $l>n$ and $m$ such that the line bundle $K_{X}^{-l} \otimes L_{X}^{m}$ is very ample and

$$
\frac{a(n) m}{2 l}<\frac{\hat{S}_{D}}{n(n-1)} .
$$

Then, there exists a complete Kähler metric $\bar{\omega}$ on $X \backslash D$ satisfies following properties:

- $\bar{\omega}$ is equivalent to $\omega_{0}$, i.e., there is a constant $C>0$ such that

$$
C^{-1} \omega_{0}<\bar{\omega}<C \omega_{0} .
$$

Moreover, the Kähler metric $\bar{\omega}$ is of asymptotically conical geometry.

- Assume that $n \geq 4$. If $\theta_{D}$ is $\csc K$ and $0<\hat{S}_{D}<n(n-1)$, the $C^{k, \alpha}$-norm of the scalar curvature $S(\bar{\omega})$ of weight $\delta \in\left(4, \min \left\{2 n, 2+2 n(n-1) / \hat{S}_{D}\right\}\right)$ can be made arbitrarily small. 
Thus, we obtain the Kähler metric $\bar{\omega}$ which is of asymptotically conical geometry. In addition, the scalar curvature of $\bar{\omega}$ is arbitrarily small in the sense of the weight norm.

Step 4. Finally, by applying the similar argument in the proof of Theorem 2 to the Kähler metric $\bar{\omega}$ obtained in Theorem 5 , we obtain our main result in this article:

Theorem 6 Assume following conditions:

- $n \geq 6$ and there is no nonzero holomorphic vector field on $X$ which vanishes on $D$.

- The following inequality holds:

$$
0<3 \hat{S}_{D}<n(n-1) .
$$

- There are positive integers $l>n$ and $m$ such that the line bundle $K_{X}^{-l} \otimes L_{X}^{m}$ is very ample and

$$
\frac{a(n) m}{2 l}<\frac{\hat{S}_{D}}{n(n-1)} .
$$

Then, if $D$ admits a cscK metric $\theta_{D}, X \backslash D$ admits a complete scalar-flat Kähler metric.

In other word, we can solve the following forth order nonlinear partial differential equation:

$$
S(\bar{\omega}+\sqrt{-1} \partial \bar{\partial} \phi)=0, \quad \bar{\omega}+\sqrt{-1} \partial \bar{\partial} \phi>0, \quad \phi \in C_{\delta-4}^{4, \alpha}
$$

for a weight $8<\delta<\min \left\{2 n, 2+2 n(n-1) / \hat{S}_{D}\right\}$. The reason why we assume that $n \geq 6$ and $0<3 \hat{S}_{D}<n(n-1)$ in Theorem 6 is that we need the isomorphic Laplacian $\Delta_{\omega}$ between higher order weighted Banach spaces.

This article is organized as follows. In Sect. 2, recalling the result due to Hwang-Singer [12], we give the volume growth of a geodesic ball with respect to the Kähler metric obtained in [12]. This case is a toy-model of our problem. In Sect. 3, we prove Theorem 1. To prove this, we use fundamental results in matrix analysis. In Sect. 4, we will introduce the asymptotically conicalness of open Riemannian manifolds and weighted Banach spaces by following Bando-Kobayashi [3]. In Sect. 5, we study the linearization of the scalar curvature operator between some weighted Banach spaces. In Sect. 6, we prove Theorem 2 by following Arezzo-Pacard [1], [2] (see also [17]). In Sect. 7, we construct Kähler potentials, i.e., strictly plurisubharmonic functions, whose scalar curvature is under control. In addition, we glue these plurisubharmonic functions by using the regularized maximum function. In Sect. 8, we prove Theorem 3. To show this, we recall the $C^{2, \varepsilon}$-estimate of a solution of the degenerate complex Monge-Ampère equation. In Sect. 9, we prove Theorem 4. In Sect. 10, we will prove Theorem 5 . Namely, we recover the asymptotically conicalness by constructing an average metric. In Sect. 11, we prove Theorem 6, i.e., we show the existence of a complete scalar-flat Kähler metric.

\section{The case of line bundles}

Before considering the general case, we consider the existence of a complete scalar-flat Kähler metrics on line bundles and compute the volume growth. Let $(X, L)$ be an $n$-dimensional polarized manifold and $\theta \in 2 \pi c_{1}(L)$ be a $\operatorname{cscK}$ metric. In this case, the value of the scalar curvature is equal to the following average value given by

$$
\hat{S}_{X}:=\frac{n c_{1}(X) \cup c_{1}(L)^{n-1}}{c_{1}(L)^{n}},
$$


where $(2 \pi)^{n} c_{1}(L)^{n}=\int_{X} \theta^{n}$ and $(2 \pi)^{n} c_{1}(X) \cup c_{1}(L)^{n-1}=\int_{X} \operatorname{Ric}(\theta) \wedge \theta^{n-1}$. Note that $\hat{S}_{X}$ is a topological invariant. In 2002, Hwang-Singer [12] showed the following:

Theorem 7 Let $(X, L)$ be an n-dimensional polarized manifold. Suppose that there exists a constant scalar curvature Kähler metric $\theta \in 2 \pi c_{1}(L)$ and the value of the scalar curvature of $\theta$ is nonnegative:

$$
\hat{S}_{X} \geq 0
$$

Then, there exists a complete scalar-flat Kähler metric $\omega$ on the total space of the dual line bundle $L^{-1}$.

Remark 1 In [12], they treat more general cases which contain the existence of a complete scalar-flat Kähler metric on the disc bundle in $L^{-1}$. In this article, it is enough for us to consider Theorem 7.

To compute the volume growth of the Kähler metric $\omega$ above, we need to recall the proof of Theorem 7 by following [12] (see also [17]).

\subsection{LeBrun-Simanca metrics}

In this subsection, we construct the LeBrun-Simanca metric for an ample line bundle. This metric for the dual of the tautological line bundle over $\mathbb{C P}^{n-1}$ was found by LeBrun and Simanca $[14,16]$ (see also [17]).

Fix an $n$-dimensional polarized manifold $(X, L)$. Consider a Hermitian metric $h$ on $L$ which defines the Kähler metric $\theta \in 2 \pi c_{1}(L)$. Let $p: L^{-1} \rightarrow X$ be the projection map and $\left(L^{-1}\right)^{*}$ be the complement of the zero section of $L^{-1}$. Define a smooth function $s$ by

$$
s:\left(L^{-1}\right)^{*} \rightarrow \mathbb{R}, \quad \xi \mapsto \log h^{-1}(\xi, \xi),
$$

where $h^{-1}$ is a Hermitian metric on $L^{-1}$ induced by $h$.

Definition 1 The LeBrun-Simanca metric $\omega$ on $\left(L^{-1}\right)^{*}$ is defined by

$$
\omega:=\sqrt{-1} \partial \bar{\partial} f(s)
$$

where $f$ is a smooth, increasing and strictly convex function on $\mathbb{R}$.

To extend $\omega$ to the whole space $L^{-1}$, we start to compute $\omega$ in local coordinates. Fix a point $z_{0}$ in the base space $X$. Then we can find a local holomorphic coordinate chart $U$ around $z_{0}$ with a local holomorphic trivialization of $L^{-1}$ around $z_{0}$ :

$$
\left.\left(L^{-1}\right)\right|_{U} \cong U \times \mathbb{C}, \quad \xi \mapsto(z, w),
$$

where $w$ is a fiber coordinate. Then, in these local coordinates, we can write as

$$
s(z, w)=\log |w|^{2}-\log h(z),
$$

where $h(z)$ is a positive function defined by some local non-vanishing holomorphic section of $L$ on $U$. For any point $z_{0} \in X$, we can choose the above trivialization so that:

$$
d \log h\left(z_{0}\right)=0 .
$$

Let us compute at a point $\left(z_{0}, w\right)$ with $w \neq 0$;

$$
\sqrt{-1} \partial \bar{\partial} f(s)=\ddot{f}(s) \sqrt{-1} \frac{d w \wedge d \bar{w}}{|w|^{2}}+\dot{f}(s) p^{*} \theta,
$$


where the symbol $\dot{f}$ denotes the differential of $f$ with respect to the variable $s$.

To simplify the construction of a scalar-flat Kähler metric on $L^{-1}$, following [17], we introduce Legendre transforms and momentum profiles and recall fundamental facts of them. By using them, we can give a condition on the extension of $\omega$ to the whole space $L^{-1}$ and compute the scalar curvature not as a nonlinear PDE in forth order but as an ODE in second order by following [12].

Definition 2 Let $f$ be a strictly convex and smooth function on $\mathbb{R}$. Set $\tau:=\dot{f}(s)$ and $I$ be an image of $\tau$. The Legendre transform $F$ on $I$ of $f$ with variable $\tau$ is defined by

$$
s \tau=f(s)+F(\tau) .
$$

Note that there are following relations:

$$
F^{\prime}(\tau)=s, \quad F^{\prime \prime}(\tau)=\frac{1}{\ddot{f}(s)},
$$

where we use the symbol $F^{\prime}(\tau)$ as the differential of $F$ with respect to the variable $\tau$.

Definition 3 Let $I \subset \mathbb{R}$ be an image of $\tau$. The momentum profile $\varphi$ of the metric $\omega=$ $\sqrt{-1} \partial \bar{\partial} f(s)$ is defined by the following:

$$
\varphi: I \rightarrow \mathbb{R}, \quad \varphi(\tau)=\frac{1}{F^{\prime \prime}(\tau)},
$$

where $F$ is the Legendre transform of $f$ defined above.

Clearly, there are following relations:

$$
\varphi(\tau)=\ddot{f}(s), \quad \frac{d \tau}{d s}=\varphi(\tau) .
$$

The following proposition is the converse of the above construction:

Proposition 1 Let $I \subset \mathbb{R}$ be any interval and $\varphi$ be a smooth positive function defined on $I$. Then we can find a smooth and strictly convex function $f$ on some interval $J$ of $\mathbb{R}$ such that

$$
\tau=\dot{f}(s), \quad \varphi(\tau)=\ddot{f}(s) .
$$

Proof Let $G=G(\tau)$ be a function on $I$ with $G^{\prime}(\tau)=1 / \varphi(\tau)$. Since $G$ is strictly monotone increasing, we have $\tau=G^{-1}(s)$. Set $J:=G(I)$. Proposition 1 is proved by setting

$$
f(s):=\int_{c}^{s} G^{-1}(t) d t
$$

for some $c \in J$.

\subsection{The extension to the total space}

In this subsection, we give a condition such that the LeBrun-Simanca metric $\omega$ can be extended to the whole space $L^{-1}$ as a Kähler metric by following [12] (see also [17]). By using the momentum profile $\varphi, \omega$ can be rewritten as follows:

$$
\omega=\varphi(\tau) \sqrt{-1} \frac{d w \wedge d \bar{w}}{|w|^{2}}+\tau p^{*} \theta .
$$


First, we set a momentum profile $\varphi$ defined on $I:=(1, N)$ for some $N \in(1, \infty]$ so that a function $f$ is defined on $J=\mathbb{R}$ in the way in the proof of Proposition 1 . Then, the formula (14) implies that the LeBrun-Simanca metric $\omega$ is positive in the base direction at any point in the zero section. Following [12], to obtain the positivity in the fiber direction and the smoothness of $\omega$ on the whole space $L^{-1}$, we pose the boundary condition on $\varphi$ :

Proposition 2 Suppose that $\varphi$ satisfies the following boundary condition:

$$
\varphi(1)=0, \quad \varphi^{\prime}(1)=1,
$$

and can be extended smoothly in a neighborhood of 1 . Then $\omega$ can be extended to $L^{-1}$ as a Kähler metric.

For simplicity, we will denote the extended metric on $L^{-1}$ by the same symbol $\omega$.

\subsection{The Ricci form and the scalar curvature}

In this subsection, we compute the Ricci form and the scalar curvature of the LeBrun-Simanca metric $\omega$ (see [12,17]).

Proposition 3 The Ricci form Ric $(\omega)$ and the scalar curvature $S(\omega)$ of $\omega$ are given by

$$
\begin{aligned}
\operatorname{Ric}(\omega) & =-\varphi\left(\varphi^{\prime}+n \frac{\varphi}{\tau}\right)^{\prime} \frac{\sqrt{-1} d w \wedge d \bar{w}}{|w|^{2}}-\left(\varphi^{\prime}+n \frac{\varphi}{\tau}\right) p^{*} \theta+p^{*} \operatorname{Ric}(\theta), \\
S(\omega) & =\frac{p^{*} S(\theta)}{\tau}-\frac{1}{\tau^{n}} \frac{d^{2}}{d \tau^{2}}\left(\tau^{n} \varphi(\tau)\right),
\end{aligned}
$$

where Ric $(\omega)$ is a pointwise formula.

Proof First, the Ricci form $\operatorname{Ric}(\omega)$ is locally given by the following:

$$
\operatorname{Ric}(\omega)=-\sqrt{-1} \partial \bar{\partial} \log \omega^{n+1} .
$$

By the direct computation at a point $z_{0}$, we have

$$
\begin{aligned}
\omega^{n+1} & =\frac{\ddot{f}(s) \dot{f}(s)^{n} p^{*} \theta^{n} \wedge \sqrt{-1} d w \wedge d \bar{w}}{|w|^{2}} \\
& =\frac{\varphi(\tau) \tau^{n} p^{*} \theta^{n} \wedge \sqrt{-1} d w \wedge d \bar{w}}{|w|^{2}} .
\end{aligned}
$$

If we choose another trivialization $(z, \hat{w})$ of $L^{-1}$, there exists a holomorphic transform function $g$ such that $\hat{w}=g(z) w$. The differential of $g(z)$ does not affect the above formula because $p^{*} \theta^{n}$ is the top wedge product in the base direction. Therefore, $\omega^{n+1}$ is invariant under the choice of the local coordinates $(z, w)$ and we can use the formula above globally.

Let us compute $\operatorname{Ric}(\omega)$ at a point $\left(z_{0}, w_{0}\right)$ :

$$
\begin{aligned}
\operatorname{Ric}(\omega) & =-\sqrt{-1} \partial \bar{\partial} \log \varphi(\tau(s))-n \sqrt{-1} \partial \bar{\partial} \log \tau(s)+p^{*} \operatorname{Ric}(\theta) \\
& =-\varphi\left(\varphi^{\prime}+n \frac{\varphi}{\tau}\right)^{\prime} \frac{\sqrt{-1} d w \wedge d \bar{w}}{|w|^{2}}-\left(\varphi^{\prime}+n \frac{\varphi}{\tau}\right) p^{*} \theta+p^{*} \operatorname{Ric}(\theta) .
\end{aligned}
$$

Note that the equation of $\operatorname{Ric}(\omega)$ is completely divided into the base direction and fiber direction. Taking a trace of the Ricci form by the metric $\omega=\varphi(\tau) \sqrt{-1} d w \wedge d \bar{w} /|w|^{2}+\tau p^{*} \theta$, 
we have the following:

$$
\begin{aligned}
S(\omega) & =-\left(\varphi^{\prime}+n \frac{\varphi}{\tau}\right)^{\prime}-\frac{n}{\tau}\left(\varphi^{\prime}+n \frac{\varphi}{\tau}\right)+\frac{p^{*} S(\theta)}{\tau} \\
& =-\frac{1}{\tau^{n}} \frac{d^{2}}{d \tau^{2}}\left(\tau^{n} \varphi(\tau)\right)+\frac{p^{*} S(\theta)}{\tau} .
\end{aligned}
$$

Thus, the proof of Proposition 3 is finished.

\subsection{ODE}

In this subsection, we prove Theorem 7 by using Proposition 3. The key of the proof is that we can consider the scalar-flat condition as the case of ordinary differential equations (ODE) in second order on the assumption that $\omega$ is $\operatorname{cscK}$.

Proof of Theorem 7 If the Kähler metric $\theta$ has a constant scalar curvature, the value of the scalar curvature $S(\theta)$ is equal to the average of the scalar curvature:

$$
\hat{S}_{X}=\frac{n c_{1}(X) \cup c_{1}(L)^{n-1}}{c_{1}(L)^{n}} .
$$

By the formula in Proposition 3, to make $\omega$ scalar-flat, it is enough to solve the following ODE with the boundary condition:

$$
\frac{d^{2}}{d \tau^{2}}\left(\tau^{n} \varphi(\tau)\right)=\hat{S}_{X} \tau^{n-1}, \quad \varphi(1)=0, \quad \varphi^{\prime}(1)=1 .
$$

In fact, a solution of this is easily given by

$$
\varphi(\tau)=\frac{\hat{S}_{X}}{n(n+1)} \tau-\left(\frac{\hat{S}_{X}}{n}-1\right) \tau^{1-n}+\left(\frac{\hat{S}_{X}}{n+1}-1\right) \tau^{-n} .
$$

If $N=\infty, \varphi=O(\tau)$ as $\tau \rightarrow \infty$. If $N<\infty, \varphi$ vanishes like a polynomial. Recall that $s=\int \varphi^{-1} d \tau$. In both cases, applying Proposition 1 for these $\varphi$, we can obtain an increasing, strictly convex and smooth function $f$ defined on $\mathbb{R}$ by setting an interval $I:=\{\tau \in \mathbb{R} \mid \varphi(\tau)$ is positive \}. Then, we have finished the proof of Theorem 7 .

\subsection{Volume growth}

In this subsection, we compute the volume growth of the Kähler metric $\omega$.

Proposition 4 Fix a point $\xi \in L^{-1}$. Suppose that $\omega$ be the LeBrun-Simanca metric as above. Let $B(\xi, r)$ be a geodesic ball with respect to $\omega$ in $L^{-1}$ of radius $r$ centered at $\xi$.

(1) If $\hat{S}_{X}>0$, we have

$$
\int_{B(\xi, r)} \omega^{n+1}=O\left(r^{2(n+1)}\right) \quad \text { as } \quad r \rightarrow \infty .
$$

(2) If $\hat{S}_{X}=0$, we have

$$
\int_{B(\xi, r)} \omega^{n+1}=O\left(r^{2}\right) \quad \text { as } \quad r \rightarrow \infty .
$$


For a point $y \in X$, a symbol $\xi_{y}$ denotes an element of the fiber $L_{y}^{-1}$. In particular, a symbol $0_{y}$ denotes the zero element of the fiber $L_{y}^{-1}$. In this section, we use the LeBrun-Simanca metric $\omega$ given by the solution (17) in the both cases of $\hat{S}_{X}>0$ and $\hat{S}_{X}=0$. First, we compute a relation between the geodesic distance for the metric $\omega$ and the Hermitian norm for $h^{-1}$. For simplicity, $\left|\xi_{y}\right|$ denotes a square root of the Hermitian norm $h^{-1}\left(\xi_{y}, \xi_{y}\right)$.

Lemma 1 (a) If $\hat{S}_{X}>0$, we have

$$
d\left(0_{y}, \xi_{y}\right)=O\left(\left|\xi_{y}\right|^{\frac{\hat{S}_{X}}{n(n+1)}}\right) \quad \text { as } \quad\left|\xi_{y}\right| \rightarrow \infty
$$

and

$$
\tau=O\left(d\left(0_{y}, \xi_{y}\right)^{2}\right) \quad \text { as } \quad\left|\xi_{y}\right| \rightarrow \infty .
$$

(b) If $\hat{S}_{X}=0$, we have

$$
d\left(0_{y}, \xi_{y}\right)=O\left(\left(\log \left|\xi_{y}\right|\right)^{\frac{n+1}{2 n}}\right) \quad \text { as } \quad\left|\xi_{y}\right| \rightarrow \infty
$$

and

$$
\tau=O\left(d\left(0_{y}, \xi_{y}\right)^{\frac{2}{n+1}}\right) \quad \text { as } \quad\left|\xi_{y}\right| \rightarrow \infty .
$$

Proof First, we prove the statement $(a)$. By the completeness of $\omega$, there exists a length minimizing geodesic connecting any pair of two points in $L^{-1}$. Fix the length minimizing geodesic $\gamma(t), t \in[0,1]$ from $0_{y}$ to $\xi_{y}$. Clearly, for fixed $y \in X$, the image of the geodesic $\gamma(t)$ is in the fiber $L_{y}^{-1}$. For simplicity, we assume that $h^{-1}\left(\xi_{y}, \xi_{y}\right)=|w|^{2}$ in the trivialization (7). Set $v=|w|$.

(1) Recall that the positive function $\varphi(\tau)$ in the case $(a)$ is written as

$$
\varphi(\tau)=\frac{\hat{S}_{X}}{n(n+1)} \tau-\left(\frac{\hat{S}_{X}}{n}-1\right) \tau^{1-n}+\left(\frac{\hat{S}_{X}}{n+1}-1\right) \tau^{-n} .
$$

Since $\tau \rightarrow \infty$ as $v \rightarrow \infty$, the second and third terms above are very small as $v \rightarrow \infty$. Then,

$$
\begin{aligned}
\frac{d \tau}{d v} & =\varphi(\tau) \frac{2}{v} \\
& =\frac{2 \hat{S}}{n(n+1)} \frac{\tau}{v}+1 . \text { o.t. }
\end{aligned}
$$

Here the symbol 1.o.t. denotes lower order terms as $v \rightarrow \infty$. We have

$$
\frac{1}{\tau} \frac{d \tau}{d v}=\frac{2 \hat{S}}{n(n+1)} \frac{1}{v}+\text { 1.o.t. }
$$

Thus, we have

$$
\tau=O\left(\left|\xi_{y}\right|^{\frac{2 \hat{S}_{X}}{n(n+1)}}\right)
$$

as $\left|\xi_{y}\right| \rightarrow \infty$.

Denote $\gamma(t)=\left(y, w_{t}\right)$. Since the LeBrun-Simanca metric $\omega$ is $S^{1}$-invariant, we can write

$$
w_{t}=\theta_{t} w_{1}
$$


where $\theta_{t}$ is some real nonnegative function such that $\theta_{0}=0$ and $\theta_{1}=1$. Since it is enough to compute for sufficiently large $\left|\xi_{y}\right|$, we have

$$
\begin{aligned}
& d\left(0_{y}, \xi_{y}\right)=\int_{0}^{1} \sqrt{\omega(\gamma \dot{(t)}, \dot{\gamma(t)})} d t \\
& =\int_{0}^{1} \sqrt{\frac{\left|\dot{w}_{t}\right|^{2}}{\left|w_{t}\right|^{2}} \varphi(\tau)} d t \\
& =\int_{0}^{1} \frac{\dot{\theta}_{t}}{\theta_{t}} \sqrt{\varphi(\tau)} d t \\
& =\int_{c}^{1}\left|w_{1}\right|^{\frac{\hat{s}}{n(n+1)}} \dot{\theta}_{t} \theta_{t}^{\frac{\hat{s}}{n(n+1)}-1} d t+\text { 1.o.t. } \\
& =O\left(\left|\xi_{y}\right|^{\frac{\hat{S}}{n(n+1)}}\right)
\end{aligned}
$$

as $\left|\xi_{y}\right| \rightarrow \infty$, where $c \in(0,1)$ is some fixed constant. Thus, the statement $(a)$ follows.

(b) Recall that the positive function $\varphi(\tau)$ in the case $(b)$ is written as

$$
\varphi(\tau)=\tau^{1-n}-\tau^{-n} .
$$

Similarly, we have

$$
\frac{d \tau}{d v}=\frac{2 \tau^{1-n}}{v}+\text { 1.o.t. }
$$

Then,

$$
\tau^{n}=O\left(\log \left|\xi_{y}\right|\right)
$$

as $\left|\xi_{y}\right| \rightarrow \infty$.

Denote $\gamma(t)=\left(y, w_{t}\right)$. Similarly, we have

$$
\begin{aligned}
d\left(0_{y}, \xi_{y}\right) & =\int_{0}^{1} \sqrt{\omega\left(\dot{\xi}_{y}^{t}, \dot{\xi}_{y}^{t}\right)} d t \\
& =\int_{c}^{1} \frac{\dot{\theta}_{t}}{\theta_{t}} \tau^{\frac{1-n}{2}} d t+\text { 1.o.t. } \\
& =A \int_{c}^{1} \frac{\dot{\theta}_{t}}{\theta_{t}}\left(\log \left|w_{1}\right|+\log \theta_{t}\right)^{\frac{1-n}{2 n}} d t+\text { l.o.t. } \\
& =O\left(\left(\log \left|\xi_{y}\right|\right)^{\frac{n+1}{2 n}}\right)
\end{aligned}
$$

as $\left|\xi_{y}\right| \rightarrow \infty$, where $c \in(0,1)$ and $A>0$ are some fixed constants. Thus, the statement $(b)$ follows.

Using Lemma 1, we prove Proposition 4.

Proof of Proposition 4 It is enough to compute the volume growth of a geodesic ball in $L^{-1}$ of radius $r$ centered at $0_{x}$. Since the restriction of $\omega$ to the zero section is $\theta$, we have

$$
\begin{aligned}
d\left(0_{x}, \xi_{y}\right) & =d\left(0_{x}, 0_{y}\right)+d\left(0_{y}, \xi_{y}\right) \\
& \leq \operatorname{diam}(X, \theta)+d\left(0_{y}, \xi_{y}\right) .
\end{aligned}
$$


Thus,

$$
-\operatorname{diam}(X, \theta)+d\left(0_{y}, \xi_{y}\right) \leq d\left(0_{x}, \xi_{y}\right) \leq \operatorname{diam}(X, \theta)+d\left(0_{y}, \xi_{y}\right) .
$$

By Stokes' theorem, we have

$$
\begin{aligned}
\int_{B\left(0_{x}, r\right)} \omega^{n+1} & =\int_{B\left(0_{x}, r\right)} \tau^{n} \varphi(\tau) \sqrt{-1} \partial s \wedge \bar{\partial} s \wedge p^{*} \theta^{n} \\
& =\frac{1}{n+1} \int_{B\left(0_{x}, r\right)} \sqrt{-1} \partial s \wedge \bar{\partial}\left(\tau^{n+1}\right) \wedge p^{*} \theta^{n} \\
& =-\frac{1}{n+1}\left(\int_{\partial B\left(0_{x}, r\right)} \tau^{n+1} \sqrt{-1} \partial s \wedge p^{*} \theta^{n}+(2 \pi)^{n+1} c_{1}(L)^{n}\right) \\
& =-C_{1} \int_{\partial B\left(0_{x}, r\right)} \tau^{n+1} \sqrt{-1} \frac{d w}{w} \wedge p^{*} \theta^{n}-C_{2},
\end{aligned}
$$

where $C_{1}$ and $C_{2}$ are positive constants depending only on $n$ and $L$.

In the case (1), previous computations (21) and (28) imply

$$
\tau^{n+1}=O\left(r^{2(n+1)}\right) .
$$

For $r>\operatorname{diam}(X, \theta)$, the residue theorem holds for each $y \in X$. Thus, we have

$$
-\int_{\partial B\left(0_{x}, r\right)} \sqrt{-1} \frac{d w}{w} \wedge p^{*} \theta^{n}=(2 \pi)^{n+1} c_{1}(L)^{n} .
$$

The formula (29) implies the first statement in Proposition 4.

In the case (2), previous computations (23) and (28) imply

$$
\tau^{n+1}=O\left(r^{2}\right) .
$$

Similarly, (31) and the formula (29) imply the second statement in Proposition 4.

\section{The higher order decay}

In this section, we prove Theorem 1 . Let $\left(X, L_{X}\right)$ be an $n$-dimensional polarized manifold. Let $h_{X}$ be a Hermitian metric on the line bundle $L_{X}$ which defines a Kähler metric $\theta_{X}$ on $X$. Then, the restriction $h_{D}$ of $h_{X}$ to a line bundle $L_{D}:=\left.L_{X}\right|_{D}$ over $D$ defines a Kähler metric $\theta_{D}$ on $D$. Let $\sigma_{D} \in H^{0}\left(X, L_{X}\right)$ be a defining section of $D$. Set $t:=\log \left\|\sigma_{D}\right\|^{-2}$, where $\left\|\sigma_{D}\right\|^{2}=h_{X}\left(\sigma_{D}, \sigma_{D}\right)$. From the construction of the complete Kähler metrics in Theorem 7 and [3], we can define a complete Kähler metric $\omega_{0}$ on $X \backslash D$ by

$$
\begin{aligned}
\omega_{0} & :=\frac{n(n-1)}{\hat{S}_{D}} \sqrt{-1} \partial \bar{\partial} \exp \left(\frac{\hat{S}_{D}}{n(n-1)} t\right) \\
& =\exp \left(\frac{\hat{S}_{D}}{n(n-1)} t\right)\left(\theta_{X}+\frac{\hat{S}_{D}}{n(n-1)} \sqrt{-1} \partial t \wedge \bar{\partial} t\right),
\end{aligned}
$$

where $\hat{S}_{D}>0$ is the average value of the scalar curvature $S\left(\theta_{D}\right)$ :

$$
\hat{S}_{D}:=\frac{\int_{D} S\left(\theta_{D}\right) \theta_{D}^{n-1}}{\int_{D} \theta_{D}^{n-1}}=\frac{(n-1) c_{1}\left(K_{D}^{-1}\right) \cup c_{1}\left(L_{D}\right)^{n-2}}{c_{1}\left(L_{D}\right)^{n-1}}
$$


By similar ways in Sect. 2, we have the followings

Lemma 2 Let $r$ be a distance function defined by $\omega_{0}$ from a fixed point $x_{0} \in X \backslash D$. Then,

$$
r(x)=O\left(\left\|\sigma_{D}\right\|^{-\frac{\hat{S}_{D}}{n(n-1)}}(x)\right)
$$

as $x \rightarrow D$.

Lemma 3 The volume growth of $\omega_{0}$ is given by

$$
\operatorname{Vol}_{\omega_{0}}\left(B\left(x_{0}, r\right)\right)=O\left(r^{2 n}\right)
$$

as $r \rightarrow \infty$.

Thus, Lemma 2 implies that it is enough to show that

$$
S\left(\omega_{0}\right)=O\left(\left\|\sigma_{D}\right\|^{2+2 \hat{S}_{D} / n(n-1)}\right)
$$

as $\sigma_{D} \rightarrow 0$.

To show Theorem 1, we have to compute $\operatorname{Ric}\left(\theta_{X}\right)$ and $\operatorname{Ric}\left(\omega_{0}\right)$. Unfortunately, we can't compute the scalar curvature of $\omega_{0}$ in the same way in the proof of Proposition 3. So, we study the determinant of $\theta_{X}$ and the inverse matrix of $\omega_{0}$. First, we recall fundamental results in matrix analysis (see [22]).

\subsection{Matrix analysis}

To compute Ricci forms of Kähler metrics $\theta_{X}, \omega_{0}$, we need the following lemma:

Lemma 4 Consider the following matrix

$$
T=\left[\begin{array}{ll}
A & B \\
C & D
\end{array}\right]
$$

where $A$ is an invertible matrix. Then, the determinant of $T$ is given by

$$
\operatorname{det} T=\operatorname{det} A \operatorname{det}\left(D-C A^{-1} B\right) .
$$

The block $D-C A^{-1} B$ is called the Schur complement of the block $D$ of the matrix $T$ (see [22, p. 23]). For the reader's convenience, we give a proof of this lemma.

Proof The result immediately follows from the following formula:

$$
\left[\begin{array}{ll}
A & B \\
C & D
\end{array}\right]=\left[\begin{array}{cc}
I & O \\
C A^{-1} & \dot{I}
\end{array}\right]\left[\begin{array}{cc}
A & O \\
O & D-C A^{-1} B
\end{array}\right]\left[\begin{array}{cc}
I & A^{-1} B \\
O & \dot{I}
\end{array}\right]
$$

where $I$ and $\dot{I}$ denote suitable identity matrices.

To take a trace with respect to the Kähler metric $\omega_{0}$, we need the following inverse matrix formula (see [22, p. 24]):

Lemma 5 Consider the following matrix

$$
T=\left[\begin{array}{ll}
A & B \\
C & D
\end{array}\right]
$$


Assume that $A$ and $S:=D-C A^{-1} B$ are invertible. Then, $T$ is invertible and the inverse matrix of $T$ can be written as

$$
T^{-1}=\left[\begin{array}{cc}
A^{-1}+A^{-1} B S^{-1} C A^{-1} & -A^{-1} B S^{-1} \\
-S^{-1} C A^{-1} & S^{-1}
\end{array}\right] .
$$

Similarly, we give a proof of this lemma for the reader's convenience.

Proof From the proof of the previous lemma, we have

$$
\begin{aligned}
{\left[\begin{array}{ll}
A & B \\
C & D
\end{array}\right]^{-1} } & =\left[\begin{array}{cc}
I & A^{-1} B \\
O & \dot{I}
\end{array}\right]^{-1}\left[\begin{array}{cc}
A & O \\
O & S
\end{array}\right]^{-1}\left[\begin{array}{cc}
I & O \\
C A^{-1} & \dot{I}
\end{array}\right]^{-1} \\
& =\left[\begin{array}{cc}
I & -A^{-1} B \\
O & \dot{I}
\end{array}\right]\left[\begin{array}{cc}
A^{-1} & O \\
O & S^{-1}
\end{array}\right]\left[\begin{array}{cc}
I & O \\
-C A^{-1} & \dot{I}
\end{array}\right] \\
& =\left[\begin{array}{cc}
A^{-1}+A^{-1} B S^{-1} C A^{-1} & -A^{-1} B S^{-1} \\
-S^{-1} C A^{-1} & S^{-1}
\end{array}\right] .
\end{aligned}
$$

\subsection{Local trivialization and normal coordinates}

Before studying the scalar curvature $S\left(\omega_{0}\right)$ near $D$, we choose a local trivialization and normal coordinates around a point of $D$.

First, fix a point $p \in D$. Since $D$ is the smooth hypersurface of $X$, there exist local holomorphic coordinates $\left(z^{1}, z^{2}, \ldots, z^{n-1}, w\right)$ centered at $p$ where $D$ is defined by $\{w=0\}$ locally and $\left(z^{1}, z^{2}, \ldots, z^{n-1}\right)$ are local holomorphic coordinates of $D$. Then, there exists a local trivialization of $L_{X}$ such that we can write as $\left\|\sigma_{D}\right\|^{2}=|w|^{2} e^{-\varphi}$ for a smooth function $\varphi$ near $p$ satisfying

$$
d \varphi(0)=0 .
$$

We may assume that if $\left(z^{1}, z^{2}, \ldots, z^{n-1}, w\right)=(0,0, \ldots, 0, w)$, we have

$$
\varphi=O\left(|w|^{2}\right) .
$$

Second, we consider the existence of normal coordinates with respect to the Kähler metric $\theta_{X}$ around $p$ preserving the condition (33). Since $\theta_{X}=\sqrt{-1} \partial \bar{\partial} t=\sqrt{-1} \partial \bar{\partial} \log \left\|\sigma_{D}\right\|^{-2}$ is the Kähler metric on $X$, in coordinates above, we can write locally as

$$
\theta_{X}=\sqrt{-1}\left(\sum_{i, j=1}^{n-1} g_{i, \bar{j}} d z^{i} \wedge d \bar{z}^{j}+\sum_{a=1}^{n-1}\left(g_{a, \bar{w}} d z^{a} \wedge d \bar{w}+g_{w, \bar{a}} d w \wedge d \bar{z}^{a}\right)+g_{w, \bar{w}} d w \wedge d \bar{w}\right) .
$$

For simplicity, write $\left(z^{1}, \ldots, z^{n-1}, w\right)=(z ; w)$. Consider another holomorphic coordinate chart $\left(\hat{z}^{1}, \ldots, \hat{z}^{n-1}, w\right)=(\hat{z} ; w)$ around $p \in D$. Directly, we have

$$
\begin{aligned}
\frac{\partial}{\partial w}\left(g_{\hat{i}, \bar{j}}\right) & =\frac{\partial}{\partial w}\left(\frac{\partial z^{k}}{\partial \hat{z}^{i}} \frac{\partial \bar{z}^{l}}{\partial \overline{\hat{z}}^{j}} g_{k, \bar{l}}\right) \\
& =\frac{\partial}{\partial w} \frac{\partial z^{k}}{\partial \hat{z}^{i}}\left(\frac{\partial \bar{z}^{l}}{\partial \overline{\bar{z}}^{j}} g_{k, \bar{l}}\right)+\frac{\partial z^{k}}{\partial \hat{z}^{i}} \frac{\partial \bar{z}^{l}}{\partial \overline{\bar{z}}^{j}} \frac{\partial g_{k, \bar{l}}}{\partial w} .
\end{aligned}
$$


Set the condition

$$
\frac{\partial z^{k}}{\partial \hat{z}^{i}}(0 ; 0)=\delta_{k, i}
$$

So, we have

$$
\frac{\partial}{\partial w}\left(g_{\hat{i}, \bar{j}}\right)(0 ; 0)=\frac{\partial}{\partial w} \frac{\partial z^{k}}{\partial \hat{z}^{i}} g_{k, \bar{j}}(0 ; 0)+\frac{\partial g_{i, \bar{j}}}{\partial w}(0 ; 0) .
$$

Considering the equation $\partial g_{\hat{i}, \overline{\hat{j}}} / \partial w(0 ; 0)=0$, we have

$$
\frac{\partial}{\partial w} \frac{\partial z^{k}}{\partial \hat{z}^{i}}(0 ; 0)=-\sum_{j} g^{k, \bar{j}}(0 ; 0) \frac{\partial g_{i, \bar{j}}}{\partial w}(0 ; 0) \text {. }
$$

Thus, we have

Lemma 6 By the change of holomorphic coordinates $(\hat{z} ; w)$ around $p \in D$ defined by

$$
z^{\alpha}=\sum_{i=1}^{n-1} \hat{z}^{i}\left(\delta_{i, \alpha}-w \sum_{j=1}^{n-1} g^{\alpha, \bar{j}}(0 ; 0) \frac{\partial g_{i, \bar{j}}}{\partial w}(0 ; 0)\right) \quad(\alpha=1,2, \ldots, n-1),
$$

we have

$$
\frac{\partial g_{\hat{i}, \bar{j}}}{\partial w}(0 ; 0)=0 .
$$

In particular, at $(\hat{z} ; w)=(0 ; w)$, we have

$$
g_{\hat{i}, \overline{\hat{j}}}(0 ; w)=g_{\hat{i}, \hat{j}}(0 ; 0)+O\left(|w|^{2}\right) .
$$

Consequently, we obtain

Proposition 5 We can find a local trivialization of $L_{X}$ and local holomorphic coordinates so that

$$
\varphi=O\left(|w|^{2}\right), \quad g_{\hat{i}, \overline{\hat{j}}}(0 ; w)=g_{\hat{i}, \overline{\hat{j}}}(0 ; 0)+O\left(|w|^{2}\right) .
$$

at $\left(\hat{z}^{1}, \ldots, \hat{z}^{n-1}, w\right)=(0, \ldots, 0, w)$.

Proof In new local coordinates above, we have

$$
\frac{\partial \varphi}{\partial \hat{z}^{i}}=\frac{\partial \varphi}{\partial z^{j}} \frac{\partial z^{j}}{\partial \hat{z}^{i}}+\frac{\partial \varphi}{\partial w} \frac{\partial w}{\partial \hat{z}^{i}},
$$

and

$$
\frac{\partial z^{j}}{\partial \hat{z}^{i}}(0 ; 0)=\delta_{i, j}, \quad \frac{\partial \varphi}{\partial z^{i}}(0 ; 0)=\frac{\partial \varphi}{\partial w}(0 ; 0)=0 .
$$

Thus, the proposition follows.

For simplicity, we write new local coordinates $\left(\hat{z}^{1}, \ldots, \hat{z}^{n-1}, w\right)$ by the same symbol $\left(z^{1}, \ldots, z^{n-1}, w\right)$. 


\subsection{Proof of Theorem 1}

Recall that $\omega_{0}$ is written as

$$
\omega_{0}=\exp \left(\frac{\hat{S}_{D}}{n(n-1)} t\right)\left(\theta_{X}+\frac{\hat{S}_{D}}{n(n-1)} \sqrt{-1} \partial t \wedge \bar{\partial} t\right)
$$

and it is enough to show that

$$
S\left(\omega_{0}\right)=O\left(\left\|\sigma_{D}\right\|^{2+2 \hat{S}_{D} / n(n-1)}\right)
$$

as $\sigma_{D} \rightarrow 0$. First, we show

Lemma 7 The Ricci form of $\omega_{0}$ is given by

$$
\operatorname{Ric}\left(\omega_{0}\right)=\operatorname{Ric}\left(\theta_{X}\right)-\frac{\hat{S}_{D}}{n-1} \theta_{X}-\sqrt{-1} \partial \bar{\partial} \log \left(1+\frac{\hat{S}_{D}}{n(n-1)}\|\partial t\|_{\theta_{X}}^{2}\right) .
$$

Proof To prove this lemma, it is enough to see the volume form of $\omega_{0}$. From the definition of $\omega_{0}$, we have

$$
\omega_{0}=\exp \left(\frac{\hat{S}_{D}}{n(n-1)} t\right)\left(\theta_{X}+\frac{\hat{S}_{D}}{n(n-1)} \sqrt{-1} \partial t \wedge \bar{\partial} t\right) .
$$

So, the following identity

$$
\sqrt{-1} \partial t \wedge \bar{\partial} t \wedge \theta_{X}^{n-1}=\frac{1}{n}\|\partial t\|_{\theta_{X}}^{2} \theta_{X}^{n}
$$

implies that the volume form of $\omega_{0}$ is given by

$$
\omega_{0}^{n}=\exp \left(\frac{\hat{S}_{D}}{n-1} t\right)\left(1+\frac{\hat{S}_{D}}{n(n-1)}\|\partial t\|_{\theta_{X}}^{2}\right) \theta_{X}^{n} .
$$

Recall that the Ricci form is given by $\operatorname{Ric}\left(\omega_{0}\right)=-\sqrt{-1} \partial \bar{\partial} \log \omega_{0}^{n}$. Thus, the lemma follows. $\square$

Thus, we easily have $S\left(\omega_{0}\right)=O\left(\left\|\sigma_{D}\right\|^{2 \hat{S}_{D} / n(n-1)}\right)$ as $\sigma_{D} \rightarrow 0$.

Firstly, we show the following proposition to prove Theorem 1.

Proposition 6 If $\theta_{D}$ is a cscK metric, we have

$$
S\left(\omega_{0}\right)=O\left(\left\|\sigma_{D}\right\|^{1+2 \hat{S}_{D} / n(n-1)}\right)
$$

as $\sigma_{D} \rightarrow 0$.

Proof To prove this, we compute the Ricci form of $\theta_{X}$. Write

$$
\theta_{X}=\left[\begin{array}{cccc}
g_{1, \overline{1}} & \cdots & g_{1, \overline{n-1}} & g_{1, \bar{w}} \\
\vdots & \ddots & \vdots & \vdots \\
g_{n-1, \overline{1}} & \cdots & g_{n-1, \overline{n-1}} & g_{n-1, \bar{w}} \\
g_{w, \overline{1}} & \cdots & g_{w, \overline{n-1}} & g_{w, \bar{w}}
\end{array}\right]=\left[\begin{array}{cc}
B & R \\
\bar{R} & W
\end{array}\right]
$$

in the previous local holomorphic coordinates. Since Lemma 4 implies that $\operatorname{det} \theta_{X}=$ $\operatorname{det} B \operatorname{det}\left(W-\bar{R}^{t} B^{-1} R\right)$, we have

$$
\operatorname{Ric}\left(\theta_{X}\right)=-\sqrt{-1} \partial \bar{\partial} \log \operatorname{det} B-\sqrt{-1} \partial \bar{\partial} \log \left(W-\bar{R}^{t} B^{-1} R\right) .
$$


Recall the notation $\left(z^{1}, \ldots, z^{n-1}, w\right)=(z ; w)$. Consider the expansion at $w=0$;

$$
\operatorname{det} B(z ; w)=\operatorname{det} B(z ; 0)+w \frac{\partial \operatorname{det} B}{\partial w}+\bar{w} \frac{\partial \operatorname{det} B}{\partial \bar{w}}+O\left(|w|^{2}\right) .
$$

Recall that

$$
\operatorname{Ric}\left(\theta_{D}\right)=-\sqrt{-1} \sum_{i, j=1}^{n-1} \frac{\partial^{2} \log \operatorname{det} B(z ; 0)}{\partial z^{i} \partial \bar{z}^{j}} d z^{i} \wedge d \bar{z}^{j},
$$

and $S\left(\theta_{D}\right)=\operatorname{tr}_{\theta_{D}} \operatorname{Ric}\left(\theta_{D}\right)=\hat{S}_{D}$. By (36),

$$
\begin{aligned}
\operatorname{Ric}\left(\theta_{X}\right)= & \operatorname{Ric}\left(\theta_{D}\right)+O(|w|) d z \wedge d \bar{z}-\sqrt{-1} \partial \bar{\partial} \log \left(W-\bar{R}^{t} B^{-1} R\right) \\
& +O(1) d w \wedge d \bar{z}+O(1) d z \wedge d \bar{w}+O(1) d w \wedge d \bar{w}
\end{aligned}
$$

at $(0 ; w)$. Here $d z$ denote differential 1 -forms in directions of $D$. To prove Proposition 6 , it is clearly enough to take the trace with respect to the metric

$$
\theta_{X}+\frac{\hat{S}_{D}}{n(n-1)} \sqrt{-1} \partial t \wedge \bar{\partial} t .
$$

For simplicity, set $a:=\hat{S}_{D} / n(n-1)>0$. Since $\partial t=\partial \varphi-d w / w$, the metric $\theta_{X}+a \sqrt{-1} \partial t \wedge$ $\bar{\partial} t$ can be written as

$$
\left[\begin{array}{cccc}
g_{1, \overline{1}}+a \varphi_{1} \varphi_{\overline{1}} & \cdots & g_{1, \overline{n-1}}+a \varphi_{1} \varphi_{\overline{n-1}} & g_{1, \bar{w}}+a \varphi_{1}\left(\varphi_{\bar{w}}-1 / \bar{w}\right) \\
\vdots & \ddots & \vdots & \vdots \\
g_{n-1, \overline{1}}+a \varphi_{n-1} \varphi_{\overline{1}} & \cdots & g_{n-1, \overline{n-1}}+a \varphi_{n-1} \varphi_{\overline{n-1}} & g_{n-1, \bar{w}}+a \varphi_{n-1}\left(\varphi_{\bar{w}}-1 / \bar{w}\right) \\
g_{w, \overline{1}}+a\left(\varphi_{w}-1 / w\right) \varphi_{\overline{1}} & \cdots & g_{w, \overline{n-1}}+a\left(\varphi_{w}-1 / w\right) \varphi_{\overline{n-1}} & g_{w, \bar{w}}+a\left(\varphi_{w}-1 / w\right)\left(\varphi_{\bar{w}}-1 / \bar{w}\right)
\end{array}\right],
$$

where $\varphi_{i}$ denotes $\partial \varphi / \partial z^{i}$. For simplicity, write the matrix above as

$$
\theta_{X}+a \sqrt{-1} \partial t \wedge \bar{\partial} t=\left[\begin{array}{cc}
E & F \\
G & H
\end{array}\right] .
$$

In order to take the trace of $\operatorname{Ric}\left(\omega_{0}\right)$ with respect to the metric $\theta_{X}+a \sqrt{-1} \partial t \wedge \bar{\partial} t$, we compute the inverse matrix of this. Since we only consider $S\left(\omega_{0}\right)$ near $D, H=O\left(|w|^{-2}\right)$ as $w \rightarrow 0$. By Lemma 5, we have

$$
\left[\begin{array}{cc}
E^{-1}+E^{-1} F S^{-1} G E^{-1} & -E^{-1} F S^{-1} \\
-S^{-1} G E^{-1} & S^{-1}
\end{array}\right],
$$

where $S:=H-G E^{-1} F$. Since $S=O\left(|w|^{-2}\right)$ as $w \rightarrow 0$, we get

$$
E^{-1} F S^{-1} G E^{-1}, E^{-1} F S^{-1}, S^{-1} G E^{-1}, S^{-1}=O\left(|w|^{2}\right) .
$$

Thus, to compute the scalar curvature $S\left(\omega_{0}\right)$, it is enough to study the block $E^{-1}+$ $E^{-1} F S^{-1} G E^{-1}$. In this case, by considering the expansion at $w=0$, we can write

$$
E=B(0 ; 0)+J,
$$

where $J=O\left(|w|^{2}\right)$. So we have

$$
\begin{aligned}
E^{-1} & =(B(0 ; 0)+J)^{-1} \\
& =B(0 ; 0)^{-1}\left(I+J B(0 ; 0)^{-1}\right)^{-1}
\end{aligned}
$$




$$
\begin{aligned}
& =B(0 ; 0)^{-1}\left(I+\sum_{i>0}\left(-J B(0 ; 0)^{-1}\right)^{i}\right) \\
& =B(0 ; 0)^{-1}+O\left(|w|^{2}\right) .
\end{aligned}
$$

Consider the term

$$
-\sqrt{-1} \partial \bar{\partial} \log \left(1+\frac{\hat{S}_{D}}{n(n-1)}\|\partial t\|_{\theta_{X}}^{2}\right),
$$

where

$$
\begin{aligned}
\|\partial t\|_{\theta_{X}}^{2}= & \sum_{i, j}^{n-1} g^{i, \bar{j}} \varphi_{i} \varphi_{\bar{j}}+\sum_{a=1}^{n-1}\left(g^{a, \bar{w}} \varphi_{a}\left(\varphi_{\bar{w}}-1 / \bar{w}\right)+g^{w, \bar{a}}\left(\varphi_{w}-1 / w\right) \varphi_{\bar{a}}\right) \\
& +g^{w, \bar{w}}\left(\varphi_{w}-1 / w\right)\left(\varphi_{\bar{w}}-1 / \bar{w}\right) .
\end{aligned}
$$

Note that $g^{w, \bar{w}}=\left(W-\bar{R}^{t} B^{-1} R\right)^{-1}$. Thus, we have

$$
\begin{aligned}
& -\sqrt{-1} \partial \bar{\partial} \log \left(W-\bar{R}^{t} B^{-1} R\right)-\sqrt{-1} \partial \bar{\partial} \log \left(1+\frac{\hat{S}_{D}}{n(n-1)}\|\partial t\|_{\theta_{X}}^{2}\right) \\
& =-\sqrt{-1} \partial \bar{\partial} \log \left(1+O\left(|w|^{2}\right)\right) .
\end{aligned}
$$

Thus,

$$
\begin{aligned}
\left\|\sigma_{D}\right\|^{-2 a} S\left(\omega_{0}\right) & =\operatorname{tr}_{\theta_{X}+a \sqrt{-1} \partial t \wedge \bar{\partial} t} \operatorname{Ric}\left(\omega_{0}\right) \\
& =\operatorname{tr}_{\theta_{X}+a \sqrt{-1} \partial t \wedge \bar{\partial} t}\left(\operatorname{Ric}\left(\theta_{D}\right)-\frac{\hat{S}_{D}}{(n-1)} \theta_{D}\right)+O(|w|)
\end{aligned}
$$

as $w \rightarrow 0$. Therefore, Proposition 6 is proved.

Remark 2 Roughly, we have proved that

$$
S\left(\omega_{0}\right)=C\left\|\sigma_{D}\right\|^{\frac{2 \hat{S}_{D}}{n(n-1)}}\left(S\left(\theta_{D}\right)-\hat{S}_{D}+O\left(\left\|\sigma_{D}\right\|\right)\right)
$$

near $D$. Thus, in fact, $\theta_{D}$ is $\operatorname{cscK}$ if and only if $S\left(\omega_{0}\right)$ has a zero along $D$ of order $1+$ $2 \hat{S}_{D} / n(n-1)$ in our construction.

Secondly, we prove Theorem 1 by altering the Hermitian metric $h_{X}$. By following BandoKobayashi [3], we take a smooth function $a \in C^{\infty}(X, \mathbb{R})$ such that $\left.a\right|_{D} \equiv 0$. Define a Hermitian metric on $L_{X}$ by

$$
h_{X, a}:=e^{-a} h_{X}
$$

Note that this modification does not change the Hermitian metric $h_{D}$ on $L_{D}$. For this Hermitian metric $h_{X, a}$, we write

$$
\left\|\sigma_{D}\right\|_{a}^{2}:=\left\|\sigma_{D}\right\|_{h_{X, a}}^{2}=e^{-a}\left\|\sigma_{D}\right\|_{h_{X}}^{2}, \quad t_{a}=\log \left\|\sigma_{D}\right\|_{a}^{-2} .
$$

In addition, we define the Kähler metrics by

$$
\theta_{X, a}:=\sqrt{-1} \partial \bar{\partial} t_{a}, \quad \omega_{a}:=\frac{n(n-1)}{\hat{S}_{D}} \sqrt{-1} \partial \bar{\partial} \exp \left(\frac{\hat{S}_{D}}{n(n-1)} t_{a}\right) .
$$


We consider the following function:

$$
\left\|\sigma_{D}\right\|_{a}^{-2 \hat{S}_{D} / n(n-1)} S\left(\omega_{a}\right) .
$$

Take a point $p \in D$ and a local holomorphic coordinate chart centered at $p$ in the previous subsection. Recall that $D=\{w=0\}$ locally. In order to prove Theorem 1, it is enough to show the following proposition:

Proposition 7 We can find a smooth function a on $X$ such that $\left.a\right|_{D} \equiv 0$ and

$$
\frac{\partial}{\partial w}\left(\left\|\sigma_{D}\right\|_{a}^{-2 \hat{S}_{D} / n(n-1)} S\left(\omega_{a}\right)\right)=0
$$

at any point $p$.

Proof By using Proposition 6, we obtain $S\left(\omega_{0}\right)=O\left(\left\|\sigma_{D}\right\|^{1+2 \hat{S}_{D} / n(n-1)}\right)$ and $S\left(\omega_{a}\right)=$ $O\left(\left\|\sigma_{D}\right\|^{1+2 \hat{S}_{D} / n(n-1)}\right)$. Recall the relation $\left\|\sigma_{D}\right\|^{2 \hat{S}_{D} / n(n-1)}=O\left(r^{-2}\right)$ as $r \rightarrow \infty$. By following [3] (see also Lemma 11 in this paper), we can find functions $F_{0}$ and $F_{a}$ on $X \backslash D$ such that

$$
\Delta_{\omega_{0}} F_{0}=S\left(\omega_{0}\right), \quad \Delta_{\omega_{a}} F_{a}=S\left(\omega_{a}\right) .
$$

Note that Proposition 6 implies that $F_{0}=O\left(\left\|\sigma_{D}\right\|\right)$ and $F_{a}=O\left(\left\|\sigma_{D}\right\|\right)$ as $\sigma_{D} \rightarrow 0$. Since $\left\|\sigma_{D}\right\|^{-2 \hat{S}_{D} / n(n-1)} S\left(\omega_{0}\right)$ and $\left\|\sigma_{D}\right\|_{a}^{-2 \hat{S}_{D} / n(n-1)} S\left(\omega_{a}\right)$ are smooth functions on $X$, so the interior Schauder estimate implies that these functions $F_{0}$ and $F_{a}$ can be extended to smooth functions on the whole space $X$. For simplicity, we denote these extended functions by the same symbols $F_{0}$ and $F_{a}$. Clearly, these functions satisfy that $\left.\left.F_{0}\right|_{D} \equiv F_{a}\right|_{D} \equiv 0$. For these functions $F_{0}, F_{a}$, we can write as follows:

$$
\begin{aligned}
\Delta_{\omega_{a}} F_{a} & =S\left(\omega_{a}\right) \\
& =\operatorname{tr}_{\omega_{a}} \operatorname{Ric}\left(\omega_{a}\right) \\
& =\operatorname{tr}_{\omega_{a}}\left(\operatorname{Ric}\left(\omega_{a}\right)-\operatorname{Ric}\left(\omega_{0}\right)\right)+\left(\operatorname{tr}_{\omega_{a}}-\operatorname{tr}_{\omega_{0}}\right) \operatorname{Ric}\left(\omega_{0}\right)+\Delta_{\omega_{0}} F_{0} \\
& =-\operatorname{tr}_{\omega_{a}} \sqrt{-1} \partial \bar{\partial} \log \left(\frac{\omega_{a}^{n}}{\omega_{0}^{n}}\right)+\left(\operatorname{tr}_{\omega_{a}}-\operatorname{tr}_{\omega_{0}}\right) \operatorname{Ric}\left(\omega_{0}\right)+\Delta_{\omega_{0}} F_{0} .
\end{aligned}
$$

To compute the first term in (37), we recall that

$$
\begin{aligned}
\omega_{a}^{n} & =\exp \left(\frac{\hat{S}_{D}}{n-1}(t+a)\right)\left(\frac{\hat{S}_{D}}{n-1} \theta_{X, a}^{n-1} \wedge \partial(t+a) \wedge \overline{\partial(t+a)}+\theta_{X, a}^{n}\right) \\
& =\frac{\hat{S}_{D}}{n-1}\left(\theta_{X}+\sqrt{-1} \partial \bar{\partial} a\right)^{n-1} \wedge \partial(t+a) \wedge \overline{\partial(t+a)}\left(1+O\left(|w|^{2}\right)\right)
\end{aligned}
$$

near $p \in D$. Since we can write $\partial t=\partial \log \left\|\sigma_{D}\right\|^{-2}=-d w / w+O(1)$ near $p$, it suffices to see the term including $|w|^{-2} d w \wedge d \bar{w}$. So, we can compute as follows:

$$
\begin{aligned}
& \log \left(\frac{\omega_{a}^{n}}{\omega_{0}^{n}}\right)-\frac{S_{D}}{n-1} a \\
& =\log \frac{\left(\theta_{X}+\sqrt{-1} \partial \bar{\partial} a\right)^{n-1} \wedge \partial(t+a) \wedge \overline{\partial(t+a)}\left(1+O\left(|w|^{2}\right)\right)}{\theta_{X}^{n-1} \wedge \partial t \wedge \overline{\partial t}\left(1+O\left(|w|^{2}\right)\right)} \\
& =\log \frac{\theta_{X}^{n-1}\left(1+\Delta_{\theta_{D}} a+O\left(|w|^{2}\right)\right) \wedge\left(1-w \frac{\partial a}{\partial w}\right) d w \wedge \overline{\left(1-w \frac{\partial a}{\partial w}\right) d w}\left(1+O\left(|w|^{2}\right)\right)}{\theta_{X}^{n-1} \wedge d w \wedge d \bar{w}\left(1+O\left(|w|^{2}\right)\right)}
\end{aligned}
$$


Thus, at $p$, we have

$$
\begin{aligned}
& \frac{\partial}{\partial w}\left(\log \left(\frac{\omega_{a}^{n}}{\omega_{0}^{n}}\right)\right) \\
= & \frac{\partial}{\partial w} \log \left(\left(1+\Delta_{\theta_{D}} a+O\left(|w|^{2}\right)\right)\left(1-w \frac{\partial a}{\partial w}\right)\left(\overline{\left.1-w \frac{\partial a}{\partial w}\right)}\right)\right)+\frac{S_{D}}{n-1} \frac{\partial a}{\partial w} \\
= & \Delta_{\theta_{D}} \frac{\partial a}{\partial w}+\left(\frac{S_{D}}{n-1}-1\right) \frac{\partial a}{\partial w} .
\end{aligned}
$$

Let us compute the second term in (37). Lemma 7 implies that the Ricci form of $\omega_{0}$ is given by

$$
\operatorname{Ric}\left(\omega_{0}\right)=\operatorname{Ric}\left(\theta_{X}\right)-\frac{\hat{S}_{D}}{n-1} \theta_{X}-\sqrt{-1} \partial \bar{\partial} \log \left(1+\frac{\hat{S}_{D}}{n(n-1)}\|\partial t\|_{\theta_{X}}^{2}\right) .
$$

Since the formula $g_{1}^{-1}-g_{2}^{-1}=g_{1}^{-1}\left(g_{2}-g_{1}\right) g_{2}^{-1}$ holds for any two invertible matrices $g_{1}$ and $g_{2}$, we can compute the term $\operatorname{tr}_{\omega_{a}}-\operatorname{tr}_{\omega_{0}}$. In particular, $\theta_{X, a}^{-1}-\theta_{X}^{-1}=-\theta_{X}^{-1}(\sqrt{-1} \partial \bar{\partial} a) \theta_{X, a}^{-1}$. By applying the computation in the proof of Proposition 6 to $\operatorname{tr}_{\omega_{a}}-\operatorname{tr}_{\omega_{0}}$, we obtain

$$
\begin{gathered}
\frac{\partial}{\partial w}\left(\left\|\sigma_{D}\right\|_{a}^{-2 \hat{S}_{D} / n(n-1)}\left(\operatorname{tr}_{\omega_{a}}-\operatorname{tr}_{\omega_{0}}\right) \operatorname{Ric}\left(\omega_{0}\right)\right) \\
=-\left(\sqrt{-1} \partial \bar{\partial} \frac{\partial a}{\partial w}, \operatorname{Ric}\left(\theta_{D}\right)-\frac{\hat{S}_{D}}{n-1} \theta_{D}\right)_{\theta_{D}}
\end{gathered}
$$

at $p \in D$. Here, we have used the fact that $a \equiv 0$ on $D$ and the term $\left\|\sigma_{D}\right\|_{a}^{-2 \hat{S}_{D} / n(n-1)}\left(\operatorname{tr}_{\omega_{a}}-\right.$ $\left.\operatorname{tr}_{\omega_{0}}\right)$ must be vanished on $D$.

Therefore, by using (38) and (39), we can compute the differential of (37) multiplied by $\left\|\sigma_{D}\right\|_{a}^{-2 \hat{S}_{D} / n(n-1)}$ with respect to $w$ as follows:

$$
\begin{aligned}
\Delta_{\theta_{D}} \frac{\partial F_{a}}{\partial w}= & \frac{\partial}{\partial w}\left(\left\|\sigma_{D}\right\|_{a}^{-2 \hat{S}_{D} / n(n-1)} S\left(\omega_{a}\right)\right) \\
= & -\Delta_{\theta_{D}}^{2} \frac{\partial a}{\partial w}-\left(\frac{\hat{S}_{D}}{n-1}-1\right) \Delta_{\theta_{D}} \frac{\partial a}{\partial w} \\
& -\left(\sqrt{-1} \partial \bar{\partial} \frac{\partial a}{\partial w}, \quad \operatorname{Ric} \theta_{D}-\frac{\hat{S}_{D}}{n-1} \theta_{D}\right)_{\theta_{D}}+\Delta_{\theta_{D}} \frac{\partial F_{0}}{\partial w} \\
= & -\Delta_{\theta_{D}}^{2} \frac{\partial a}{\partial w}-\left(\sqrt{-1} \partial \bar{\partial} \frac{\partial a}{\partial w}, \quad \operatorname{Ric} \theta_{D}\right)_{\theta_{D}}+\Delta_{\theta_{D}} \frac{\partial a}{\partial w}+\Delta_{\theta_{D}} \frac{\partial F_{0}}{\partial w}
\end{aligned}
$$

Here, we have used the fact that $\left(\sqrt{-1} \partial \bar{\partial}(\partial a / \partial w), \theta_{D}\right)_{\theta_{D}}=\Delta_{\theta_{D}}(\partial a / \partial w)$. Recall that the linearization of the scalar curvature operator satisfies

$$
L_{\theta_{D}} \varphi=-\Delta_{\theta_{D}}^{2} \varphi-\left(\sqrt{-1} \partial \bar{\partial} \varphi, \quad \operatorname{Ric} \theta_{D}\right)_{\theta_{D}}=-\mathscr{D}_{\theta_{D}}^{*} \mathscr{D}_{\theta_{D}} \varphi+\left(\nabla^{1,0} \varphi, \nabla^{0,1} S\left(\theta_{D}\right)\right)_{\theta_{D}}
$$

for $\varphi \in C^{\infty}(D)$ (see [17]). So, we have 


$$
\begin{aligned}
\frac{\partial}{\partial w}\left(\left\|\sigma_{D}\right\|_{a}^{-2 \hat{S}_{D} / n(n-1)} S\left(\omega_{a}\right)\right) & =L_{\theta_{D}} \frac{\partial a}{\partial w}+\Delta_{\theta_{D}} \frac{\partial a}{\partial w}+\Delta_{\theta_{D}} \frac{\partial F_{0}}{\partial w} \\
& =-\mathscr{D}_{\theta_{D}}^{*} \mathscr{D}_{\theta_{D}} \frac{\partial a}{\partial w}+\Delta_{\theta_{D}} \frac{\partial a}{\partial w}+\Delta_{\theta_{D}} \frac{\partial F_{0}}{\partial w} .
\end{aligned}
$$

Here, we have used the fact that the Kähler metric $\theta_{D}$ is a $\operatorname{cscK}$ metric on $D$. Note that $\partial F_{0} / \partial w$ defines a smooth global section of the line bundle $L_{D}^{-1}$ (equivalently, the conormal bundle $N_{D}^{-1}$ on $D$ ) and $\Delta_{\theta_{D}}$ is the Laplacian with respect to the Kähler metric $\theta_{D}$ and the corresponding Hermitian metric on $L_{D}^{-1}$ defined by $\left.h_{X}\right|_{L_{D}}$. For this Laplacian, we have $\Delta_{\theta_{D}} \leq-(n-1)$. Thus, we can solve the following differential equation:

$$
-\mathscr{D}_{\theta_{D}}^{*} \mathscr{D}_{\theta_{D}} \frac{\partial a}{\partial w}+\Delta_{\theta_{D}} \frac{\partial a}{\partial w}+\Delta_{\theta_{D}} \frac{\partial F_{0}}{\partial w}=\Delta_{\theta_{D}} \frac{\partial F_{a}}{\partial w}=0,
$$

since the operator $-\mathscr{D}_{\theta_{D}}^{*} \mathscr{D}_{\theta_{D}}+\Delta_{\theta_{D}}$ is negative and self-adjoint. By the same way in [3, p. 176], we can show the existence of a smooth function $a \in C^{\infty}(X, \mathbb{R})$ such that $\left.a\right|_{D} \equiv 0$ and $\theta_{X, a}=\theta_{X}+\sqrt{-1} \partial \bar{\partial} a>0$ on $X$.

Remark 3 In [3, p. 176], if $\theta_{D}$ is a Ricci-positive Kähler-Einstein metric, the background Kähler metric $\omega_{0}$ can be chosen so that the Ricci potential of $\omega_{0}$ decays at a higher order by altering the Hermitian metric $h_{X}$ on $K_{X}^{-1 / \alpha}$. In order to find the Hermitian metric above, they solved the following differential equation:

$$
\Delta_{\theta_{D}} \frac{\partial a}{\partial w}+(\alpha-2) \frac{\partial a}{\partial w}+\frac{\partial F_{0}}{\partial w}=0 .
$$

Here, we have used the notations in this article. In the case of Bando-Kobayashi [3], the Kähler metric $\theta_{D}$ is a Kähler Einstein metric, i.e., $\operatorname{Ric}\left(\theta_{D}\right)=(\alpha-1) \theta_{D}$, so this Eq. (41) is equivalent to the Eq. (40) by considering the image of the operator $-\Delta_{\theta_{D}}$ of (41). Therefore, our modification of the Hermitian metric $h_{X}$ can be considered as a generalization of the modification in Bando-Kobayashi [3].

From now on, let us write the modified Hermitian metric $h_{X, a}$ as $h_{X}$ for simplicity. So, we use the simple symbols $t, \theta_{X}, \omega_{0}$ from now on. Thus, we have $S\left(\omega_{0}\right)=$ $O\left(\left\|\sigma_{D}\right\|^{2+2 \hat{S}_{D} / n(n-1)}\right)$ on the assumption that $\theta_{D}$ is cscK.

\section{Asymptotically conical geometry}

Recall that the Kähler metric defined by

$$
\begin{aligned}
\omega_{0} & =\frac{n(n-1)}{\hat{S}_{D}} \sqrt{-1} \partial \bar{\partial} \exp \left(\frac{\hat{S}_{D}}{n(n-1)} t\right) \\
& =\exp \left(\frac{\hat{S}_{D}}{n(n-1)} t\right)\left(\theta_{X}+\frac{\hat{S}_{D}}{n(n-1)} \sqrt{-1} \partial t \wedge \bar{\partial} t\right)
\end{aligned}
$$

is complete on $X \backslash D$. Set $r(x):=d\left(x, x_{0}\right)$, where $d$ is the distance function from some fixed point $x_{0} \in X \backslash D$ defined by $\omega_{0}$. Following [3], the Riemannian manifold $\left(X \backslash D, \omega_{0}\right)$ is of asymptotically conical geometry which is the analytic framework in this article.

Definition 4 A complete Riemannian metric $g$ on an open manifold $M$ of dimension $m$ is said to be of $C^{k, \alpha}$-asymptotically conical geometry if for each point $p \in M$ with distance $r$ 
from a fixed point $o \in M$, there exists a harmonic coordinate system $x=\left(x^{1}, x^{2}, \cdots, x^{m}\right)$ centered at $p$ which satisfies the following conditions:

- The coordinate $x$ runs over a unit ball $B_{p}^{m} \subset \mathbb{R}^{m}$.

- If we write $g=\sum g_{i, j}(x) d x^{i} d x^{j}$, then the matrix $\left(r^{2}+1\right)^{-1} g_{i, j}(x)$ is bounded from below by a constant positive matrix independent of $p$.

- The $C^{k, \alpha}$-norms of $\left(r^{2}+1\right)^{-1} g_{i, j}(x)$ are uniformly bounded.

In particular, we simply say that $(M, g)$ is of asymptotically conical geometry if $(M, g)$ is of $C^{k, \alpha}$-asymptotically conical geometry for any $k \in \mathbb{Z}_{\geq 0}$ and $\alpha \in(0,1)$.

Definition 5 Assume that a Riemannian manifold $(M, g)$ is of asymptotically conical geometry. The $C^{k, \alpha}$-norm of a function $u$ of weight $\delta \in \mathbb{R}$ is defined by

$$
\|u\|_{C_{\delta}^{k, \alpha}}:=\sup _{p \in M}\left(r(p)^{2}+1\right)^{\delta / 2}\|u\|_{C^{k, \alpha}\left(B_{p}\right)} .
$$

The Banach space $C_{\delta}^{k, \alpha}$ is defined by the set of functions $u$ such that $\|u\|_{C_{\delta}^{k, \alpha}}<\infty$. In the above definition, we use the coordinates $x \in B_{p}^{m}$ centered at $p$ with $d(o, p)=r$ in the definition of the asymptotically conicalness.

\section{Forth order elliptic linear operators}

To prove Theorem 2, we study the linearization of the scalar curvature operator. For a smooth function $\varphi$ on $X \backslash D$, set $\omega_{t}:=\omega_{0}+t \sqrt{-1} \partial \bar{\partial} \varphi$. Recall that $S\left(\omega_{t}\right)=g_{t}^{i, \bar{j}} R_{t, i, \bar{j}}$. Thus, the linearization of the scalar curvature operator is defined by

$$
\begin{aligned}
L_{\omega_{0}}(\phi) & :=\left.\frac{d}{d t}\right|_{t=0} S\left(\omega_{t}\right) \\
& =-\Delta_{\omega_{0}}^{2} \varphi-g^{i, \bar{q}} \varphi_{p, \bar{q}} g^{p, \bar{j}} R_{j, \bar{i}} \\
& =-\Delta_{\omega_{0}}^{2} \varphi-R^{i, \bar{j}} \varphi_{i, \bar{j}} .
\end{aligned}
$$

Set $M:=X \backslash D$. The following operator plays an important role in this article.

Definition 6 The operator $\mathscr{D}_{\omega_{0}}$ is defined by

$$
\begin{aligned}
\mathscr{D}_{\omega_{0}}: C_{\delta}^{k, \alpha}(M, \mathbb{C}) & \rightarrow C_{\delta+2}^{k-2, \alpha}\left(M, \Omega^{0,1} M \otimes T^{1,0} M\right) \\
\varphi & \mapsto \bar{\partial}\left(\nabla^{1,0} \varphi\right)
\end{aligned}
$$

Here $\bar{\partial}$ is the $(0,1)$-part of the Levi-Civita connection and $\nabla^{1,0}$ is the $(1,0)$-gradient with respect to $\omega_{0}$. We call $\mathscr{D}_{\omega_{0}}^{*} \mathscr{D}_{\omega_{0}}$ the Lichnerowicz operator and we have

Lemma 8 The Lichnerowicz operator $\mathscr{D}_{\omega_{0}}^{*} \mathscr{D}_{\omega_{0}}$ satisfies

$$
\mathscr{D}_{\omega_{0}}^{*} \mathscr{D}_{\omega_{0}} \varphi=\Delta_{\omega_{0}}^{2} \varphi+R^{i, \bar{j}} \varphi_{i, \bar{j}}+\left(\nabla^{1,0} \varphi, \nabla^{0,1} S\left(\omega_{0}\right)\right)_{\omega_{0}} .
$$

Thus, we have

$$
L_{\omega_{0}}=-\mathscr{D}_{\omega_{0}}^{*} \mathscr{D}_{\omega_{0}}+\left(\nabla^{1,0} *, \nabla^{0,1} S\left(\omega_{0}\right)\right)_{\omega_{0}}
$$


The idea of proving Theorem 2 follows from Arezzo-Pacard [1] and [2] (see also [17]). Consider the following expansion:

$$
S\left(\omega_{0}+\sqrt{-1} \partial \bar{\partial} \phi\right)=S\left(\omega_{0}\right)+L_{\omega_{0}}(\phi)+Q_{\omega_{0}}(\phi) .
$$

To solve the following equation;

$$
S\left(\omega_{0}+\sqrt{-1} \partial \bar{\partial} \phi\right)=0,
$$

we will find a following fixed point:

$$
\phi=-L_{\omega_{0}}^{-1}\left(S\left(\omega_{0}\right)+Q_{\omega_{0}}(\phi)\right) .
$$

When we prove Theorem 2, we assume that $L_{\omega_{0}}$ is invertible. Therefore we need to prove that the operator

$$
\mathscr{N}(\phi):=-L_{\omega_{0}}^{-1}\left(S\left(\omega_{0}\right)+Q_{\omega_{0}}(\phi)\right)
$$

is a contraction on some Banach space.

In particular, we mainly use the weighted Banach spaces $C_{\delta-4}^{4, \alpha}(X \backslash D)$ and $C_{\delta}^{0, \alpha}(X \backslash D)$. From the definition of the weighted Banach space and local formulae of these operators, we easily have

Lemma 9 Following three operators

$$
L_{\omega_{0}}, \quad \mathscr{D}_{\omega_{0}}^{*} \mathscr{D}_{\omega_{0}}, \quad \Delta_{\omega_{0}}^{2}: C_{\delta-4}^{4, \alpha}(X \backslash D) \rightarrow C_{\delta}^{0, \alpha}(X \backslash D)
$$

are bounded.

First, we study the square of the Laplacian operator $\Delta_{\omega_{0}}^{2}$. Define a barrier function $\rho$ on $X \backslash D$ by

$$
\rho:=\exp \left(\frac{\hat{S}_{D}}{2 n(n-1)} t\right)=\left\|\sigma_{D}\right\|^{-\hat{S}_{D} / n(n-1)} .
$$

Note that for $\delta>0, \rho$ satisfies

$$
\begin{aligned}
& \Delta_{\omega_{0}} \rho^{-\delta} \\
& =\operatorname{tr}_{\omega_{0}} \sqrt{-1} \partial \bar{\partial} \exp \left(\frac{-\delta \hat{S}_{D}}{2 n(n-1)} t\right) \\
& =\frac{-\delta \hat{S}_{D}}{2 n(n-1)} \operatorname{tr}_{\omega_{0}}\left(\exp \left(\frac{-\delta \hat{S}_{D}}{2 n(n-1)} t\right)\left(\sqrt{-1} \partial \bar{\partial} t+\frac{-\delta \hat{S}_{D}}{2 n(n-1)} \sqrt{-1} \partial t \wedge \bar{\partial} t\right)\right) \\
& =\frac{-\delta \hat{S}_{D}}{2 n(n-1)} \rho^{-\delta-2} \operatorname{tr}_{\omega_{0}}\left(\exp \left(\frac{\hat{S}_{D}}{n(n-1)} t\right)\left(\sqrt{-1} \partial \bar{\partial} t+\frac{-\delta \hat{S}_{D}}{2 n(n-1)} \sqrt{-1} \partial t \wedge \bar{\partial} t\right)\right) \\
& =\frac{-\delta \hat{S}_{D}}{2 n(n-1)} \rho^{-\delta-2} \operatorname{tr}_{\omega_{0}}\left(\omega_{0}+\frac{-(\delta+2) \hat{S}_{D}}{2 n(n-1)} \exp \left(\frac{\hat{S}_{D}}{n(n-1)} t\right) \sqrt{-1} \partial t \wedge \bar{\partial} t\right) \\
& =\frac{-\delta \hat{S}_{D}}{2 n(n-1)} \rho^{-\delta-2}\left(n+\operatorname{tr}_{\omega_{0}}\left(\frac{-(\delta+2) \hat{S}_{D}}{2 n(n-1)} \exp \left(\frac{\hat{S}_{D}}{n(n-1)} t\right) \sqrt{-1} \partial t \wedge \bar{\partial} t\right)\right) \\
& \leq \frac{-\delta \hat{S}_{D}}{2 n(n-1)} \rho^{-\delta-2}\left(n-\frac{\delta+2}{2}\right)
\end{aligned}
$$


Here we have used the following inequality:

$$
\omega_{0} \geq \frac{\hat{S}_{D}}{n(n-1)} \exp \left(\frac{\hat{S}_{D}}{n(n-1)} t\right) \sqrt{-1} \partial t \wedge \bar{\partial} t .
$$

From Lemma 3, we have known that the volume growth of $\omega_{0}$ is given by

$$
\operatorname{Vol}_{\omega_{0}}\left(B\left(x_{0}, r\right)\right)=O\left(r^{2 n}\right) .
$$

In addition, we have known that $\left\|\operatorname{Ric}\left(\omega_{0}\right)\right\|_{\omega_{0}}=O\left(r^{-2}\right)$ as $r \rightarrow \infty$. From [11, Theorem 1.2], we have

Lemma 10 Set $\gamma:=n /(n-1)$. Then the following Sobolev inequality holds, i.e., there exists a constant $C>0$ such that

$$
\left(\int_{X \backslash D}|v|^{2 \gamma} \omega_{0}^{n}\right)^{1 / \gamma} \leq C \int_{X \backslash D}|\partial v|^{2} \omega_{0}^{n}
$$

for any compactly supported smooth function $v$ on $X \backslash D$.

Then, we can apply the Moser's iteration to obtain the $C_{\delta}^{0}$-estimate. Following [3, p. 178], we have

Lemma 11 If $2<\delta<2 n$, the Laplacian $\Delta_{\omega_{0}}: C_{\delta-2}^{k, \alpha}(X \backslash D) \rightarrow C_{\delta}^{k-2, \alpha}(X \backslash D)$ is isomorphic.

Recall that the standard theorem on Banach spaces (see [21, p. 77]).

Theorem 8 Let $\mathscr{X}$ and $\mathscr{Y}$ be Banach spaces. Assume that $L: \mathscr{X} \rightarrow \mathscr{Y}$ is a bounded and isomorphic linear operator. Then, the inverse $L^{-1}$ is also bounded.

Thus, the inverse of the Laplacian $\Delta_{\omega_{0}}^{-1}$ is bounded. In addition, recall the definition of Fredholm operators (see [9, Chapter $1, \S 1.4]$ ):

Definition 7 We say that a bounded linear operator $L: \mathscr{X} \rightarrow \mathscr{Y}$ between Banach spaces $\mathscr{X}$ and $\mathscr{Y}$ is a Fredholm operator if the $\operatorname{dim}(\operatorname{Ker} L)$ and $\operatorname{dim}(\operatorname{Coker} L)$ are finite and $\operatorname{Im} L$ is a closed linear subspace of $\mathscr{Y}$. For such an operator $L$, we define an index of $L$ by

$$
\operatorname{ind}(L):=\operatorname{dim}(\operatorname{Ker} L)-\operatorname{dim}(\operatorname{Coker} L) .
$$

Thus, immediately we obtain

Lemma 12 If $2<\delta<2 n$, the Laplacian $\Delta_{\omega_{0}}: C_{\delta-2}^{k, \alpha}(X \backslash D) \rightarrow C_{\delta}^{k-2, \alpha}(X \backslash D)$ is a Fredholm operator whose index ind $\left(\Delta_{\omega_{0}}\right)$ is zero. Moreover, there exists a bounded inverse $\Delta_{\omega_{0}}^{-1}$ which is also a Fredholm operator whose index is zero.

Next, we study the Lichnerowicz operator $\mathscr{D}_{\omega_{0}}^{*} \mathscr{D}_{\omega_{0}}$.

Lemma 13 Assume that $\delta>4$ and there is no nonzero holomorphic vector field on $X$ which vanishes on D. Then, the Lichnerowicz operator

$$
\mathscr{D}_{\omega_{0}}^{*} \mathscr{D}_{\omega_{0}}: C_{\delta-4}^{4, \alpha}(X \backslash D) \rightarrow C_{\delta}^{0, \alpha}(X \backslash D)
$$

is injective. 
Proof Assume that $\phi \in C_{\delta-4}^{4, \alpha}(X \backslash D)$ satisfies $\mathscr{D}_{\omega_{0}}^{*} \mathscr{D}_{\omega_{0}} \phi=0$. Integrating by parts, we have

$$
0=\int_{X \backslash D} \phi \mathscr{D}_{\omega_{0}}^{*} \mathscr{D}_{\omega_{0}} \phi \omega_{0}^{n}=\int_{X \backslash D}\left|\mathscr{D}_{\omega_{0}} \phi\right|^{2} \omega_{0}^{n} .
$$

Since $\bar{\partial} \nabla^{1,0} \phi=\mathscr{D}_{\omega_{0}} \phi=0, \nabla^{1,0} \phi$ is a holomorphic vector field on $X \backslash D$. By writing locally $\omega_{0}=\sqrt{-1} g_{i, \bar{j}} d z^{i} \wedge d \bar{z}^{j}$, the $(1,0)$-gradient of $\phi$ can be written as

$$
\nabla^{1,0} \phi=g^{i, j} \frac{\partial \phi}{\partial \bar{z}^{j}} \frac{\partial}{\partial z^{i}} .
$$

So, all coefficients $g^{i, \bar{j}} \partial \phi / \partial \bar{z}^{j}$ are holomorphic. Moreover, the definition of $\phi$ and the asymptotically conicalness imply differentials of $\phi$ and factors $g^{i, \bar{j}}$ decay near $D$. Thus, $\nabla^{1,0} \phi$ can be extended holomorphically to $X$ and vanishes on $D$. The hypothesis implies that $\phi$ is constant. Since $\phi$ decays near $D$, we have $\phi=0$ and conclude that $\mathscr{D}_{\omega_{0}}^{*} \mathscr{D}_{\omega_{0}}$ is injective.

Recall the following fundamental fact (see [9, Chapter 1, §1.4]).

Theorem 9 Let $L: \mathscr{X} \rightarrow \mathscr{Y}$ be a bounded linear operator between Banach spaces $\mathscr{X}$ and $\mathscr{Y}$. Then, $L$ is Fredholm if and only if there exists a bounded linear operator $H: \mathscr{Y} \rightarrow \mathscr{X}$ such that operators $I_{\mathscr{X}}-H \circ L$ and $I_{\mathscr{Y}}-L \circ H$ are compact. Moreover, $H$ is also Fredholm and satisfies

$$
\operatorname{ind}(L)=-\operatorname{ind}(H) \text {. }
$$

Then, we can show the following.

Lemma 14 If $4<\delta<2 n$, the Lichnerowicz operator $\mathscr{D}_{\omega_{0}}^{*} \mathscr{D}_{\omega_{0}}: C_{\delta-4}^{k, \alpha}(X \backslash D) \rightarrow$ $C_{\delta}^{k-4, \alpha}(X \backslash D)$ is a Fredholm operator whose index $\operatorname{ind}\left(\mathscr{D}_{\omega_{0}}^{*} \mathscr{D}_{\omega_{0}}\right)$ is zero.

Proof Recall the equation

$$
\mathscr{D}_{\omega_{0}}^{*} \mathscr{D}_{\omega_{0}} \phi=\Delta_{\omega_{0}}^{2} \phi+R^{j, \bar{i}} \phi_{i, \bar{j}}+\left(\nabla^{1,0} \phi, \nabla^{0,1} S\left(\omega_{0}\right)\right)_{\omega_{0}} .
$$

Since $\left(\Delta_{\omega_{0}}^{2}\right)^{-1}: C_{\delta}^{k-4, \alpha}(X \backslash D) \rightarrow C_{\delta-4}^{k, \alpha}(X \backslash D)$ is bounded, it is continuous. Consider the linear operator

$$
I_{C_{\delta-4}^{k, \alpha}(X \backslash D)}-\left(\Delta_{\omega_{0}}^{2}\right)^{-1} \circ \mathscr{D}_{\omega_{0}}^{*} \mathscr{D}_{\omega_{0}} .
$$

From the equation above, we obtain

$$
\left(I_{C_{\delta-4}^{k, \alpha}(X \backslash D)}-\left(\Delta_{\omega_{0}}^{2}\right)^{-1} \circ \mathscr{D}_{\omega_{0}}^{*} \mathscr{D}_{\omega_{0}}\right) \phi=\left(\Delta_{\omega_{0}}^{2}\right)^{-1}\left(R^{j, \bar{i}} \phi_{i, \bar{j}}+\left(\nabla^{1,0} \phi, \nabla^{0,1} S\left(\omega_{0}\right)\right)_{\omega_{0}}\right)
$$

for any $\phi \in C_{\delta-4}^{k, \alpha}$. Since $\phi \in C_{\delta-4}^{k, \alpha}(X \backslash D)$, the Arzela-Ascoli theorem implies that the operator

$$
\phi \rightarrow R^{j, \bar{i}} \phi_{i, \bar{j}}+\left(\nabla^{1,0} \phi, \nabla^{0,1} S\left(\omega_{0}\right)\right)_{\omega_{0}}
$$

is compact. The fact that $\left(\Delta_{\omega_{0}}^{2}\right)^{-1}$ is continuous implies that $I_{C_{\delta-4}^{k, \alpha}(X \backslash D)}-\left(\Delta_{\omega_{0}}^{2}\right)^{-1} \circ \mathscr{D}_{\omega_{0}}^{*} \mathscr{D}_{\omega_{0}}$ is also compact. Similarly, we obtain the compactness of the operator

$$
I_{C_{\delta}^{k-4, \alpha}(X \backslash D)}-\mathscr{D}_{\omega_{0}}^{*} \mathscr{D}_{\omega_{0}} \circ\left(\Delta_{\omega_{0}}^{2}\right)^{-1} .
$$

From Theorem 9, we have finished the proof. 
Then, Lemmas 13 and 14 imply that

Proposition 8 If $4<\delta<2 n$ and there is no nonzero holomorphic vector field on $X$ which vanishes on D, the Lichnerowicz operator $\mathscr{D}_{\omega_{0}}^{*} \mathscr{D}_{\omega_{0}}: C_{\delta-4}^{k, \alpha}(X \backslash D) \rightarrow C_{\delta}^{k-4, \alpha}(X \backslash D)$ is isomorphic and has a bounded inverse.

Thus, there exists $K>0$ such that

$$
\left\|\left(\mathscr{D}_{\omega_{0}}^{*} \mathscr{D}_{\omega_{0}}\right)^{-1}\right\|_{C_{\delta}^{0, \alpha} \rightarrow C_{\delta-4}^{4, \alpha}}<K^{-1} .
$$

In the next section, we study the operator $L_{\omega_{0}}=-\mathscr{D}_{\omega_{0}}^{*} \mathscr{D}_{\omega_{0}}+\left(\nabla^{1,0} *, \nabla^{0,1} S\left(\omega_{0}\right)\right)_{\omega_{0}}$.

Remark 4 We can show that if the $C_{2}^{1, \alpha}$-norm of $S\left(\omega_{0}\right)$ is sufficiently small, there exists the bounded inverse of $L_{\omega_{0}}$ satisfying

$$
\left\|L_{\omega_{0}}^{-1}\right\|_{C_{\delta}^{0, \alpha} \rightarrow C_{\delta-4}^{4, \alpha}}<\hat{K}^{-1}
$$

for some $\hat{K}>0$ (Condition 1$)$.

\section{Proof of Theorem 2}

This section also follows from Arezzo-Pacard [1,2] (see also [17]). Since we assume that

$$
0<\hat{S}_{D}<n(n-1),
$$

we can choose a weight $\delta$ so that

$$
\delta \in\left(4, \min \left\{2 n, 2+2 n(n-1) / \hat{S}_{D}\right\}\right)
$$

Note that if $\theta_{D}$ is cscK, Theorem 1 implies that $S\left(\omega_{0}\right)=O\left(r^{-\delta}\right)$. In addition, from Lemma 2, we can choose $\delta$ sufficiently close to $\min \left\{2 n, 2+2 n(n-1) / \hat{S}_{D}\right\}$ so that a function

$$
\phi \mathscr{D}_{\omega_{0}}^{*} \mathscr{D} \omega_{0} \phi
$$

is integrable for $\phi \in C_{\delta-4}^{4, \alpha}$ with respect to the volume form $\omega_{0}^{n}$. Hereafter, we fix a weight $\delta$ satisfying (44) and (45).

Remark 5 If $\left(D, L_{D}\right)=\left(\mathbb{P}^{n-1}, \mathscr{O}(1)\right)$, the equality above holds, i.e., $\hat{S}_{D}=n(n-1)$.

We will show that the operator $\mathscr{N}: C_{\delta-4}^{4, \alpha}(X \backslash D) \rightarrow C_{\delta-4}^{4, \alpha}(X \backslash D)$ defined in (43) has a fixed point under Conditions 1 and 2 . First, we have

Lemma 15 There exists $c_{0}>0$ depending only on $\omega_{0}$ such that if $\|\phi\|_{C_{-2}^{4, \alpha}(X \backslash D)} \leq c_{0}$, we have

$$
\left\|L_{\omega_{\phi}}-L_{\omega_{0}}\right\|_{C_{\delta-4}^{4, \alpha} \rightarrow C_{\delta}^{0, \alpha}} \leq \hat{K} / 2
$$

and $\omega_{\phi}=\omega_{0}+\sqrt{-1} \partial \bar{\partial} \phi$ is positive.

Proof Note that

$$
g_{\phi}^{-1}-g^{-1}=g_{\phi}^{-1}\left(g-g_{\phi}\right) g^{-1}
$$

for $\phi$ such that $\omega_{\phi}=\omega_{0}+\sqrt{-1} \partial \bar{\partial} \phi$ is positive. 
Locally, we can write as

$$
L_{\omega_{\phi}} \psi=-\Delta_{\omega_{\phi}}^{2} \psi-R_{\omega_{\phi}}^{i, \bar{j}} \psi_{i, \bar{j}}
$$

For instance, we have

$$
\begin{aligned}
& \left\|\left(r^{2}+1\right)^{\delta / 2}\left(g_{\phi}^{i, \bar{j}} g_{\phi}^{k, \bar{l}}-g^{i, \bar{j}} g^{k, \bar{l}}\right) \psi_{i, \bar{j}, k, \bar{l}}\right\|_{C^{0, \alpha}} \\
& \leq\left\|\left(r^{2}+1\right)^{4 / 2}\left(g_{\phi}^{i, \bar{j}} g_{\phi}^{k, \bar{l}}-g^{i, \bar{j}} g^{k, \bar{l}}\right)\right\|_{C^{0, \alpha}}\|\psi\| \|_{C_{\delta-4}^{4, \alpha}(X \backslash D)} \\
& \quad=\left\|\left(r^{2}+1\right)^{4 / 2}\left(g_{\phi}^{i, \bar{j}}\left(g_{\phi}^{k, \bar{l}}-g^{k, \bar{l}}\right)+\left(g_{\phi}^{i, \bar{j}}-g^{i, \bar{j}}\right) g^{k, \bar{l}}\right)\right\|_{C^{0, \alpha}}\|\psi\|_{C_{\delta-4}^{4, \alpha}(X \backslash D)} .
\end{aligned}
$$

Note that $g_{\phi}^{i, \bar{j}}=O\left(r^{-2}\right)$ as $r \rightarrow \infty$, if $c_{0}$ is sufficiently small. Thus, if $c_{0}$ is sufficiently small, the Eq. (46) implies that the term above can be made small arbitrarily. Applying the same argument to remainders in (47), we can use the asymptotically conicalness to obtain the desired result.

To show that the operator $\mathscr{N}: C_{\delta-4}^{4, \alpha} \rightarrow C_{\delta-4}^{4, \alpha}$ is a contraction, we need the following lemma:

\section{Lemma 16 Assume that}

$$
\|\phi\|_{C_{\delta-4}^{4, \alpha}}, \quad\|\psi\|_{C_{\delta-4}^{4, \alpha}} \leq c_{0} .
$$

Then, we have

$$
\|\mathscr{N}(\phi)-\mathscr{N}(\psi)\|_{C_{\delta-4}^{4, \alpha}} \leq \frac{1}{2}\|\phi-\psi\|_{C_{\delta-4}^{4, \alpha}}
$$

Proof Since the operator $\mathscr{N}$ is defined by $\mathscr{N}(\phi):=-L_{\omega_{0}}^{-1}\left(S\left(\omega_{0}\right)+Q_{\omega_{0}}(\phi)\right)$, we have

$$
\mathscr{N}(\phi)-\mathscr{N}(\psi)=-L_{\omega_{0}}^{-1}\left(Q_{\omega_{0}}(\phi)-Q_{\omega_{0}}(\psi)\right) .
$$

The mean value theorem implies that there exists $\chi=t \phi+(1-t) \psi$ for $t \in[0,1]$ such that

$$
D Q_{\omega_{0}, \chi}(\phi-\psi)=Q_{\omega_{0}}(\phi)-Q_{\omega_{0}}(\psi),
$$

and the direct computation implies that

$$
D Q_{\omega_{0}, \chi}=L_{\omega_{\chi}}-L_{\omega_{0}} .
$$

We know that $\|\phi\|_{C_{-2}^{4, \alpha}} \leq\|\phi\|_{C_{\delta-4}^{4, \alpha}} \leq c_{0}$. Using Lemma 15, we finish the proof.

The following Proposition implies that the existence of a complete scalar-flat Kähler metric.

Proposition 9 Set

$$
\mathscr{U}:=\left\{\phi \in C_{\delta-4}^{4, \alpha}:\|\phi\|_{C_{\delta-4}^{4, \alpha}} \leq c_{0}\right\} .
$$

If Conditions 1 and 2 hold, the operator $\mathscr{N}$ is a contraction on $\mathscr{U}$ and $\mathscr{N}(\mathscr{U}) \subset \mathscr{U}$. 
Proof By the condition $\left.\|\phi\|\right|_{C_{\delta-4}^{4, \alpha}} \leq c_{0}$, Lemmas 15 and 16 obviously imply that $\|\phi\|_{C_{-2}^{4, \alpha}} \leq$ $c_{0}, \mathscr{N}$ is a contraction on $\mathscr{U}$. Immediately, we have

$$
\|\mathscr{N}(\phi)\|_{C_{\delta-4}^{4, \alpha}} \leq\|\mathscr{N}(\phi)-\mathscr{N}(0)\|_{C_{\delta-4}^{4, \alpha}}+\left.\|\mathscr{N}(0)\|\right|_{C_{\delta-4}^{4, \alpha}} .
$$

From the previous lemma, we obtain:

$$
\|\mathscr{N}(\phi)-\mathscr{N}(0)\|_{C_{\delta-4}^{4, \alpha}} \leq \frac{1}{2} c_{0} .
$$

The hypothesis in this proposition implies that

$$
\|\mathscr{N}(0)\|_{C_{\delta-4}^{4, \alpha}} \leq \hat{K}^{-1}\left\|S\left(\omega_{0}\right)\right\|_{C_{\delta}^{0, \alpha}} \leq \frac{1}{2} c_{0} .
$$

Thus, $\mathscr{N}(\phi) \in \mathscr{U}$.

Proof of Theorem 2 If Conditions 1 and 2 hold, Proposition 9 implies that there is a unique $\phi_{\infty}:=\lim _{i \rightarrow \infty} \mathscr{N}^{i}(\phi)$ for any $\phi \in \mathscr{U} \subset C_{\delta-4}^{4, \alpha}$ satisfying $\phi_{\infty}=\mathscr{N}\left(\phi_{\infty}\right)$. Therefore, $\omega_{0}+\sqrt{-1} \partial \bar{\partial} \phi_{\infty}$ is a complete scalar-flat Kähler metric on $X \backslash D$.

\section{Plurisubharmonic functions with small scalar curvature}

To prove Theorem 4, we prepare Kähler potentials, i.e., strictly plurisubharmonic functions, whose scalar curvature is under control.

\subsection{Kähler potential near $D$}

In this subsection, we consider a Kähler potential near $D$ and study the scalar curvature of it. Recall that

$$
t=\log \left\|\sigma_{D}\right\|^{-2}
$$

and $\theta_{X}=\sqrt{-1} \partial \bar{\partial} t=\sqrt{-1} \partial \bar{\partial} \log \left\|\sigma_{D}\right\|^{-2}$ on $X \backslash D$. Set

$$
\Theta(t)=\frac{n(n-1)}{\hat{S}_{D}} \exp \left(\frac{\hat{S}_{D}}{n(n-1)} t\right) .
$$

Following [3], we can define a complete Kähler metric by

$$
\omega_{0}:=\sqrt{-1} \partial \bar{\partial} \Theta(t)=\frac{n(n-1)}{\hat{S}_{D}} \sqrt{-1} \partial \bar{\partial} \exp \left(\frac{\hat{S}_{D}}{n(n-1)} t\right)
$$

on $X \backslash D$. Recall the asymptotic behavior of the scalar curvature of $\omega_{0}$.

Lemma 17 The scalar curvature $S\left(\omega_{0}\right)$ can be estimated as follows:

$$
S\left(\omega_{0}\right)=O\left(\left\|\sigma_{D}\right\|^{2 \hat{S}_{D} / n(n-1)}\right)
$$

as $\sigma_{D} \rightarrow 0$.

Remark 6 Moreover, from Theorem 1, if $\theta_{D}$ is cscK, we have the following strong result:

$$
S\left(\omega_{0}\right)=O\left(\left\|\sigma_{D}\right\|^{2+2 \hat{S}_{D} / n(n-1)}\right)
$$

as $\sigma_{D} \rightarrow 0$. 


\subsection{Kähler potential near $F$}

In this subsection, we construct a Kähler metric on $X$ whose scalar curvature is small near the smooth hypersurface $F \in\left|K_{X}^{-l} \otimes L_{X}^{m}\right|$. Here, $l, m$ are positive integers such that the line bundle $K_{X}^{-l} \otimes L_{X}^{m}$ is very ample. For a fixed Hermitian metric on $K_{X}^{-l} \otimes L_{X}^{m}$, set $b:=\log \left\|\sigma_{F}\right\|^{-2}$. Since the holomorphic line bundle $K_{X}^{-l} \otimes L_{X}^{m}$ is very ample, we may assume that $\sqrt{-1} \partial \bar{\partial} b$ is a Kähler metric on $X$. For parameters $v>0$ and $\beta \in \mathbb{Z}_{>0}$, define a function by

$$
G_{v}^{\beta}(b):=\int_{b_{0}}^{b}\left(\frac{1}{e^{-y}+v}\right)^{1 / \beta} d y
$$

for some fixed $b_{0} \in \mathbb{R}$. Note that $G_{v}^{\beta}(b)$ is defined smoothly outside $F$ and $\lim _{b \rightarrow \infty} G_{v}^{\beta}(b)=$ $+\infty$ for any $v>0$.

Lemma 18 For $\mathbb{Z} \ni \beta \geq 1, \gamma_{v}^{\beta}:=\sqrt{-1} \partial \bar{\partial} G_{v}^{\beta}(\beta b)$ defines a Kähler metric on $X$.

Proof In fact,

$$
\begin{aligned}
\sqrt{-1} \partial \bar{\partial} G_{v}^{\beta}(\beta b) & =\beta \sqrt{-1} \partial\left[\left(\frac{1}{e^{-\beta b}+v}\right)^{1 / \beta} \bar{\partial} b\right] \\
& =\left(\frac{1}{e^{-\beta b}+v}\right)^{1 / \beta}\left(\beta \sqrt{-1} \partial \bar{\partial} b+\frac{e^{-\beta b}}{e^{-\beta b}+v} \sqrt{-1} \partial \wedge \wedge \bar{\partial} b\right) .
\end{aligned}
$$

Note that the last term

$$
\frac{e^{-\beta b}}{e^{-\beta b}+v} \sqrt{-1} \partial b \wedge \bar{\partial} b
$$

is defined smoothly on $X$ from the assumption that $\mathbb{Z} \ni \beta \geq 1$. Since $\sqrt{-1} \partial \bar{\partial} b$ is a Kähler metric on $X$, we finish the proof.

Next, the scalar curvature of $\gamma_{v}^{\beta}$ is given by

Lemma 19 For $\beta \geq 3$, we obtain

$$
S\left(\gamma_{v}^{\beta}\right)=S\left(\sqrt{-1} \partial \bar{\partial} G_{v}^{\beta}(\beta b)\right)=O\left(\left(\left\|\sigma_{F}\right\|^{2 \beta}+v\right)^{1 / \beta}\right)
$$

as $\left\|\sigma_{F}\right\| \rightarrow 0$.

Proof This lemma follows from the similar way in the computation of the scalar curvature of $\omega_{0}$. In fact, since

$$
\left(\left(\sqrt{-1} \partial \bar{\partial} G_{v}^{\beta}(\beta b)\right)^{n}=\beta^{n}\left(\frac{1}{e^{-\beta b}+v}\right)^{n / \beta}\left(1+\frac{e^{-\beta b}}{\beta\left(e^{-\beta b}+v\right)}\|\partial b\|^{2}\right)(\sqrt{-1} \partial \bar{\partial} b)^{n},\right.
$$

we have

$$
\begin{aligned}
\operatorname{Ric}\left(\sqrt{-1} \partial \bar{\partial} G_{v}^{\beta}(\beta b)\right) \\
=\operatorname{Ric}(\sqrt{-1} \partial \bar{\partial} b)-\sqrt{-1} \partial \bar{\partial} \log \left(1+\frac{e^{-\beta b}}{\beta\left(e^{-\beta b}+v\right)}\|\partial b\|^{2}\right) \\
\quad+\frac{n}{\beta}\left(\frac{1}{e^{-\beta b}+v} \sqrt{-1} \partial \bar{\partial} e^{-\beta b}+\frac{\beta}{\left(e^{-\beta b}+v\right)^{2}} \sqrt{-1} \partial e^{-\beta b} \wedge \bar{\partial} e^{-\beta b}\right) .
\end{aligned}
$$


Note that second and last terms above are zero on $F$. Thus, when we consider the scalar curvature $S\left(\gamma_{v}^{\beta}\right)$, it is enough to see the term $1 /\left(e^{-\beta b}+v\right)^{1 / \beta} \sqrt{-1} \partial \bar{\partial} b$ and the Ricci form $\operatorname{Ric}(\sqrt{-1} \partial \bar{\partial} b)$. Therefore the desired result is obtained.

Remark 7 If the value of the function $e^{-\beta b}=\left\|\sigma_{F}\right\|^{2 \beta}$ is compatible with $v$, i.e., $\left\|\sigma_{F}\right\|^{2 \beta} \approx v$, we have the following estimate of $S\left(\sqrt{-1} \partial \bar{\partial} G_{v}^{\beta}(\beta b)\right)$ :

$$
S\left(\sqrt{-1} \partial \bar{\partial} G_{v}^{\beta}(\beta b)\right)=O(1) .
$$

However, we will consider the case that $\left\|\sigma_{F}\right\|^{2 \beta} \approx v^{k}$ for sufficiently large $k \in \mathbb{N}$ which will be specified later. Namely, it suffices to consider a sufficiently small neighborhood of $F$ defined by the inequality $\left\|\sigma_{F}\right\|^{2 \beta} \leq v^{k}$ and Lemma 19 holds on this region.

\subsection{Ricci-flat Kähler metric away from $D \cup F$}

In this subsection, we study an incomplete Ricci-flat Kähler metric away from the support of the divisor $D+F$. Recall the setting in Theorem 4. Let $l>n$ and $m$ be positive integers such that there exists a holomorphic section $\sigma_{F} \in H^{0}\left(K_{X}^{-l} \otimes L_{X}^{m}\right)$ which defines a smooth hypersurface $F \subset X$, i.e., $\left(\sigma_{F}\right)_{0}=F$. It follows from the hypothesis of the average value $\hat{S}_{D}$ of the scalar curvature that divisors $D$ and $F$ intersect to each other. Set

$$
\xi:=\sigma_{F} \otimes \sigma_{D}^{-m} .
$$

Note that $\xi$ is a meromorphic section of $K_{X}^{-l}$. Then, define a singular and degenerate volume form $V$ by

$$
V:=\xi^{-1 / l} \wedge \overline{\xi^{-1 / l}}
$$

From the construction above, $V$ has finite volume on $X$ and its curvature form, i.e., the Ricci form, is zero on the complement of $D \cup F$. For the Kähler metric $\theta_{X}$ on $X$, write

$$
V=f \theta_{X}^{n}
$$

for some non-negative function $f$ on $X$ with the normalized condition

$$
\int_{X} V=\int_{X} f \theta_{X}^{n}=\int_{X} \theta_{X}^{n}
$$

We know that $f$ is smooth away from $D \cup F$. From the result due to Yau [20, Theorem 7], recall the solvability of a meromorphic complex Monge-Ampère equation:

Theorem 10 Let $L_{1}$ and $L_{2}$ be holomorphic line bundles over a compact Kähler manifold $\left(X, \theta_{X}\right)$. Let $s_{1}$ and $s_{2}$ be nonzero holomorphic sections of $L_{1}$ and $L_{2}$, respectively. Let $F$ be a smooth function on $X$ such that $\int_{X}\left|s_{1}\right|^{2 k_{1}}\left|s_{2}\right|^{-2 k_{2}} \exp (F) \theta_{X}^{n}=\operatorname{Vol}(X)$, where $k_{1} \geq 0$ and $k_{2} \geq 0$. Suppose that $\int_{X}\left|s_{2}\right|^{-2 n k_{2}}<\infty$ for $n=\operatorname{dim} X$. Then, we can solve the following equation

$$
\left(\theta_{X}+\sqrt{-1} \partial \bar{\partial} \varphi\right)^{n}=\left|s_{1}\right|^{2 k_{1}}\left|s_{2}\right|^{-2 k_{2}} \exp (F) \theta_{X}^{n}
$$

so that $\varphi$ is smooth outside divisors of $s_{1}$ and $s_{2}$ with $\sup _{X} \varphi<+\infty$.

Then, we can solve the following complex Monge-Ampère equation

$$
\left(\theta_{X}+\sqrt{-1} \partial \bar{\partial} \varphi\right)^{n}=f \theta_{X}^{n}=\xi^{-1 / l} \wedge \overline{\xi^{-1 / l}} .
$$


with $\varphi \in C^{\infty}(X \backslash D \cup F)$. Thus, we obtain a Ricci-flat Kähler metric $\theta_{X}+\sqrt{-1} \partial \bar{\partial} \varphi$ on the complement of $D \cup F$. For this solution $\varphi$, we obtain the following a priori estimate due to Kołodziej [13] (see also [10]):

Theorem 11 If $f$ is in $L^{p}\left(\theta_{X}^{n}\right)$ for some $p>1$, we have

$$
\operatorname{Osc}_{X} \varphi \leq C
$$

for some $C>0$ depending only on $\theta_{X}$ and $\|f\|_{L^{p}}$.

\subsection{Gluing plurisubharmonic functions}

In this subsection, following [6, Chapter I], we consider gluing Kähler potentials, i.e., plurisubharmonic functions, obtained in previous subsections. Let $\rho \in C^{\infty}(\mathbb{R}, \mathbb{R})$ be a nonnegative function with support in $[-1,1]$ such that $\int_{\mathbb{R}} \rho(h) d h=1$ and $\int_{\mathbb{R}} h \rho(h) d h=0$.

Lemma 20 (the regularized maximum) For arbitrary $\eta=\left(\eta_{1}, \ldots, \eta_{p}\right) \in(0,+\infty)^{p}$, the function

$$
M_{\eta}\left(t_{1}, \ldots, t_{p}\right)=\int_{\mathbb{R}^{p}} \max \left\{t_{1}+h_{1}, \ldots, t_{p}+h_{p}\right\} \prod_{1 \leq j \leq p} \eta_{j}^{-1} \rho\left(h_{j} / \eta_{j}\right) d h_{1} \ldots d h_{p}
$$

called the regularized maximum possesses the following properties :

(a) $M_{\eta}\left(t_{1}, \ldots, t_{p}\right)$ is non decreasing in all variables, smooth and convex on $\mathbb{R}^{p}$;

(b) $\max \left\{t_{1}, \ldots, t_{p}\right\} \leq M_{\eta}\left(t_{1}, \ldots, t_{p}\right) \leq \max \left\{t_{1}+\eta_{1}, \ldots, t_{p}+\eta_{p}\right\}$;

(c) $M_{\eta}\left(t_{1}, \ldots, t_{p}\right)=M_{\left(\eta_{1}, \ldots, \hat{\eta_{j}}, \ldots, \eta_{p}\right)}\left(t_{1}, \ldots, \hat{t_{j}}, \ldots, t_{p}\right)$ if $t_{j}+\eta_{j} \leq \max _{k \neq j}\left\{t_{k}-\eta_{k}\right\}$;

(d) $M_{\eta}\left(t_{1}+a, \ldots, t_{p}+a\right)=M_{\eta}\left(t_{1}, \ldots, t_{p}\right)+a$;

(e) if $u_{1}, \ldots, u_{p}$ are plurisubharmonic and satisfy $H\left(u_{j}\right)_{z}(\xi) \geq \gamma_{z}(\xi)$ where $z \mapsto \gamma_{z}$ is a continuous hermitian form on $T M$, then $u=M_{\eta}\left(u_{1}, \ldots, u_{p}\right)$ is a plurisubharmonic and satisfies $H u_{z}(\xi) \geq \gamma_{z}(\xi)$.

Remark 8 Lemma 20 is a key in the proof of Richberg theorem (see [6, p. 43]). In our case, we have already prepared three plurisubharmonic functions and must compute the Ricci form of the glued Kähler metric later. Therefore, we need the explicit formula of the glued function.

In addition, we obtain

Lemma 21 There exists a constant $C>0$ such that

$$
\left|\frac{\partial^{|\alpha|} M_{\eta}}{\partial t^{\alpha}}(t)\right| \leq C \min \left\{\eta_{j} \mid \alpha_{j} \neq 0\right\} \prod_{\alpha_{i} \neq 0} \eta_{i}^{-\alpha_{i}}
$$

for any multi index $\alpha=\left(\alpha_{i}\right)_{i}$ with $1 \leq|\alpha| \leq 4$.

Recall that the Kähler potential of $\omega_{0}$ is given by

$$
\Theta(t)=\frac{n(n-1)}{\hat{S}_{D}} \exp \left(\frac{\hat{S}_{D}}{n(n-1)} t\right) .
$$

For $\kappa \in(0,1)$, set

$$
\tilde{G}_{v}^{\beta}(b):=G_{v}^{\beta}(\beta b)+\kappa \Theta(t) .
$$

This constant $\kappa$ will be specified later. For this Kähler potential, we have 
Lemma 22 For the complete Kähler metric $\sqrt{-1} \partial \bar{\partial} \tilde{G}_{v}^{\beta}(b)$ on $X \backslash D$, we have

$$
S\left(\sqrt{-1} \partial \bar{\partial}\left(\tilde{G}_{v}^{\beta}(b)\right)\right)= \begin{cases}O\left(\left\|\sigma_{D}\right\|^{2 \hat{S}_{D} / n(n-1)}\right) & \text { near } D, \\ O\left(\left(\left\|\sigma_{F}\right\|^{2 \beta}+v\right)^{1 / \beta}\right) & \text { near } F .\end{cases}
$$

Proof First, we study the behavior of the scalar curvature near $D$. Since

$$
\left\|\sigma_{D}\right\|^{2+2 \hat{S} /(n-1)}\left(\sqrt{-1} \partial \bar{\partial}\left(\tilde{G}_{v}^{\beta}(b)\right)\right)^{n}
$$

is a smooth volume form on $X$, the Ricci form of $\sqrt{-1} \partial \bar{\partial}\left(\tilde{G}_{v}^{\beta}(b)\right)$ given by

$$
\begin{aligned}
& \operatorname{Ric}\left(\sqrt{-1} \partial \bar{\partial}\left(\tilde{G}_{v}^{\beta}(b)\right)\right. \\
& =-\left(\frac{\hat{S}}{n-1}+1\right) \theta_{X}-\sqrt{-1} \partial \bar{\partial} \log \left\|\sigma_{D}\right\|^{2+2 \hat{S} /(n-1)}\left(\sqrt{-1} \partial \bar{\partial}\left(\tilde{G}_{v}^{\beta}(b)\right)\right)^{n}
\end{aligned}
$$

is defined smoothly on X. Recall that

$$
\sqrt{-1} \partial \bar{\partial}\left(\tilde{G}_{v}^{\beta}(b)\right)=\kappa \omega_{0}+\gamma_{v}^{\beta} .
$$

As $\omega_{0}$ is of asymptotically conical geometry, we have the desired result near $D$. Similarly, the volume form

$$
\left(\left\|\sigma_{F}\right\|^{2 \beta}+v\right)^{n / \beta}\left(\sqrt{-1} \partial \bar{\partial}\left(\tilde{G}_{v}^{\beta}(b)\right)\right)^{n}
$$

is smooth near $F \backslash(D \cap F)$. Then, the following identity

$$
\begin{aligned}
\operatorname{Ric}\left(\sqrt{-1} \partial \bar{\partial}\left(\tilde{G}_{v}^{\beta}(b)\right)=\right. & \frac{n}{\beta}\left(\frac{1}{e^{-\beta b}+v} \sqrt{-1} \partial \bar{\partial} e^{-\beta b}+\frac{\beta}{\left(e^{-\beta b}+v\right)^{2}} \sqrt{-1} \partial e^{-\beta b} \wedge \bar{\partial} e^{-\beta b}\right) \\
& -\sqrt{-1} \partial \bar{\partial} \log \left(\left\|\sigma_{F}\right\|^{2 \beta}+v\right)^{n / \beta}\left(\sqrt{-1} \partial \bar{\partial}\left(\tilde{G}_{v}^{\beta}(b)\right)\right)^{n}
\end{aligned}
$$

implies the desired result near $F$.

In summary, we have prepared the three strictly plurisubharmonic functions $\Theta(t)=$ $\left(n(n-1) / \hat{S}_{D}\right) \exp \left(\left(\hat{S}_{D} / n(n-1)\right) t\right), \tilde{G}_{v}^{\beta}(b)=G_{v}^{\beta}(\beta b)+\kappa \Theta(t), t+\varphi=\log \left\|\sigma_{D}\right\|^{-2}+\varphi$ whose scalar curvature is under control. From Lemma 20, we immediately have

Proposition 10 For parameters $c, v, \eta$ and $\kappa \in(0,1)$, a function defined by

$$
M_{c, v, \eta}:=M_{\eta}\left(\Theta(t), \tilde{G}_{v}^{\beta}(b), t+\varphi+c\right)
$$

is a strictly plurisubharmonic function on $X \backslash(D \cup F)$. Here, the functions above are defined in (48), (49), (50), (51) and (52).

Remark 9 From a priori estimate due to Kołodziej [13], the solution $\varphi$ is bounded on $X$. Thus, by taking $c>0$ sufficiently large, $\varphi$ can be ignored when we consider the value of $M_{c, v, \eta}$.

By taking a sufficiently large $c>0$, we have

$$
M_{c, v, \eta}= \begin{cases}\Theta(t) & \text { near D and away from } F, \\ \tilde{G}_{v}^{\beta}(b) & \text { near } F \text { and away from } D, \\ t+\varphi+c & \text { away from } F \text { and } D .\end{cases}
$$

Set

$$
\omega_{c, v, \eta}:=\sqrt{-1} \partial \bar{\partial} M\left(\Theta(t), \tilde{G}_{v}^{\beta}(b), t+\varphi+c\right) .
$$


The reason why we consider the second Kähler potential which contains the term $\kappa \Theta(t)$ is that we want to make $\omega_{c, v, \eta}$ complete on $X \backslash D$. The function $M_{c, v, \eta}$ is defined on $X \backslash(D \cup F)$. On the other hand, Lemma 18 implies that $\omega_{c, v, \eta}$ is defined on $X \backslash D$ since the Kähler metric $\gamma_{v}^{\beta}$ is a smooth Kähler metric on $X$. From (54), we know that the scalar curvature of $\omega_{c, v, \eta}$ is small on three regions above (in particular, away from $D$ and $F, S\left(\omega_{c, v, \eta}\right)=0$ since $t+\varphi+c$ is a Kähler potential whose Ricci form is zero).

The explicit formula of $\omega_{c, v, \eta}$ is written as

$$
\begin{aligned}
\omega_{c, v, \eta}= & \frac{\partial M_{c, v, \eta}}{\partial t_{1}} \omega_{0}+\frac{\partial M_{c, v, \eta}}{\partial t_{2}}\left(\gamma_{v}^{\beta}+\kappa \omega_{0}\right)+\frac{\partial M_{c, v, \eta}}{\partial t_{3}} \sqrt{-1} \partial \bar{\partial}(t+\varphi) \\
& +\left[\partial \Theta(t) \partial \tilde{G}_{v}^{\beta}(b) \partial(t+\varphi)\right]\left[\frac{\partial^{2} M_{c, v, \eta}}{\partial t_{i} \partial t_{j}}\right]\left[\bar{\partial} \Theta(t) \bar{\partial} \tilde{G}_{v}^{\beta}(b) \bar{\partial}(t+\varphi)\right]^{t} .
\end{aligned}
$$

Thus, when we compute the scalar curvature of $\omega_{c, v, \eta}$, higher order derivatives of $\varphi$ arise in the components of the Ricci tensor of $\omega_{c, v, \eta}$. So, we must study the behavior of higher order derivatives of $\varphi$ near $D \cup F$.

\section{Proof of Theorem 3}

In this section, we prove Theorem 3. Firstly, we use the $C^{2}$-estimate due to Păun [15] (see also [7], [10, p. 366, Theorem 14.3]) for the solution $\phi$ of the complex Monge-Ampère equation (51) in the previous section to obtain the estimate of the ellipticity. i.e., the maximal ratio of the maximal eigenvalue to the minimal eigenvalue, of the Kähler metric $\theta_{X}+\sqrt{-1} \partial \bar{\partial} \varphi$. Secondly, we study how the $C^{2, \varepsilon}$-estimate of $\varphi$ depends on the ellipticity of $\theta_{X}+\sqrt{-1} \partial \bar{\partial} \varphi$ on a fixed relatively compact domain in $X \backslash(D \cup F)$. Finally, we estimate the higher order derivatives of $\varphi$ by using the Schauder estimate.

\subsection{The $C^{2}$-estimate}

To study the behavior of the higher order derivatives of $\varphi$, the elliptic operator defined by the Kähler metric $\theta_{X}+\sqrt{-1} \partial \bar{\partial} \varphi$ plays an important role. To obtain the ellipticity of $\theta_{X}+\sqrt{-1} \partial \bar{\partial} \varphi$, we use the $C^{2}$-estimate due to Păun [15] (see also [7], [10, p. 366, Theorem $14.3])$.

Theorem 12 Let $d V$ be a smooth volume form. Assume that $\varphi \in \operatorname{PSH}\left(X, \theta_{X}\right)$ satisfies

$$
\left(\theta_{X}+\sqrt{-1} \partial \bar{\partial} \varphi\right)^{n}=e^{\psi_{+}-\psi_{-}} d V
$$

with $\int_{X} \varphi \theta_{X}^{n}=0$. Here, $\psi_{+}, \psi_{-}$are quasi-plurisubharmonic functions on $X$. Assume that we are given $C>0$ and $p>1$ such that

(i) $\sqrt{-1} \partial \bar{\partial} \psi_{+} \geq-C \theta_{X}$ and $\sup _{X} \psi_{+} \leq C$.

(ii) $\sqrt{-1} \partial \bar{\partial} \psi_{-} \geq-C \theta_{X}$ and $\left\|e^{-\psi_{-}}\right\|_{L^{p}} \leq C$.

Then there exists $A>0$ depending only on $\theta_{X}, p$ and $C$ such that

$$
0 \leq \theta_{X}+\sqrt{-1} \partial \bar{\partial} \varphi \leq A e^{-\psi_{-}} \theta_{X} .
$$

Set $\psi_{+}:=\log \left\|\sigma_{D}\right\|^{2 m / l}$ and $\psi_{-}:=\log \left\|\sigma_{F}\right\|^{2 / l}$. Then, Theorem 12 implies the following inequality

$$
0 \leq \theta_{X}+\sqrt{-1} \partial \bar{\partial} \varphi \leq A\left\|\sigma_{F}\right\|^{-2 / l} \theta_{X} .
$$


Recall that the singular and degenerate volume form

$$
\left(\theta_{X}+\sqrt{-1} \partial \bar{\partial} \varphi\right)^{n}=\xi^{-1 / l} \wedge \overline{\xi^{-1 / l}}, \quad \xi=\sigma_{F} \otimes \sigma_{D}^{-m}
$$

vanishes along $D$ with order $2 m / l$ and has a pole along $F$ of order $2 / l$. So, we obtain the behavior of the product of the eigenvalues of the Kähler metric $\theta_{X}+\sqrt{-1} \partial \bar{\partial} \varphi$. From (55) and (56), we can estimate the eigenvalues of $\theta_{X}+\sqrt{-1} \partial \bar{\partial} \varphi$. Namely, the maximal eigenvalue $\Lambda$ and the minimal eigenvalue $\lambda$ of the Kähler metric $\theta_{X}+\sqrt{-1} \partial \bar{\partial} \varphi$ are estimated as follows:

$$
\Lambda=O\left(\left\|\sigma_{F}\right\|^{-2 / l}\right), \quad \lambda^{-1}=O\left(\left\|\sigma_{D}\right\|^{-2 m / l}\right) .
$$

In the next subsection, to consider the third and the forth order derivatives, we recall the $C^{2, \varepsilon}$-estimate of $\varphi$.

\subsection{The $C^{2, \varepsilon}$-estimate}

This subsection follows from [10, Chapter 14]. In this subsection, we study the relation between the ellipticity of $\theta_{X}+\sqrt{-1} \partial \bar{\partial} \varphi$ and the $C^{2, \varepsilon}$-estimate of $\varphi$. This subsection is the core of the proof of Theorem 3 because the estimate of the higher order derivatives of the solution $\varphi$ are obtained by the $C^{2, \varepsilon}$-estimate and the Schauder estimate.

Let $\mathscr{H}$ be the set of $n \times n$ Hermitian matrices. The set of positive matrices is defined by $\mathscr{H}_{+}:=\{A \in \mathscr{H} \mid A>0\}$. In addition, for $0<\lambda<\Lambda<\infty$, let $S(\lambda, \Lambda)$ be the subset of $\mathscr{H}_{+}$ whose eigenvalues lie in the interval $[\lambda, \Lambda]$. First, we recall the following result from linear algebra (see [8, p. 454, Lemma 17.13], [10, p. 372, Lemma 14.10]):

Lemma 23 We can find unit vectors $\zeta_{1}, \ldots, \zeta_{N} \in \mathbb{C}^{n}$ and $0<\lambda_{*}<\Lambda_{*}<\infty$, depending only on $n, \lambda$ and $\Lambda$, such that every $A \in S(\lambda, \Lambda)$ can be written as

$$
A=\sum_{k=1}^{N} \beta_{k} \zeta_{k} \otimes \overline{\zeta_{k}}, \quad \text { i.e., } \quad a_{i, \bar{j}}=\sum_{k} \beta_{k} \zeta_{k i} \bar{\zeta}_{k j}
$$

where $\beta_{k} \in\left[\lambda_{*}, \Lambda_{*}\right]$. The vectors $\zeta_{1}, \ldots, \zeta_{N} \in \mathbb{C}^{n}$ can be chosen so that they contain a given orthonormal basis of $\mathbb{C}^{n}$.

Remark 10 In the proof of Lemma 23, they use the following covering

$$
U\left(\zeta_{1}, \ldots, \zeta_{n^{2}}\right)=\left\{\sum_{k} \beta_{k} \zeta_{k} \otimes \bar{\zeta}_{k} \quad \mid \quad 0<\beta_{k}<2 \Lambda\right\}
$$

of the compact subset $S(\lambda / 2, \Lambda)$ (see [8, p. 454, Lemma 17.13], [10, p. 372, Lemma 14.10]). Here, $\zeta_{1}, \ldots, \zeta_{n^{2}} \in \mathbb{C}^{n}$ are unit vectors such that the matrices $\zeta_{k} \otimes \bar{\zeta}_{k}$ span $\mathscr{H}$ over $\mathbb{R}$. Thus, it follows from the form of the covering $U\left(\zeta_{1}, \ldots, \zeta_{n^{2}}\right)$ that the number $N$ in Lemma 23 is depending only on the dimension $n$. In particular, $N$ is independent of the ellipticity of $\theta_{X}+\sqrt{-1} \partial \bar{\partial} \varphi$.

Take local holomorphic coordinates $\left(z^{i}\right)_{i=1}^{n}=\left(z^{1}, z^{2}, \ldots, z^{n-2}, w_{F}, w_{D}\right)$ such that $\left\{w_{F}=0\right\}=F$ and $\left\{w_{D}=0\right\}=D$. On this coordinate chart, we can write $t=$ $a+\log \left|w_{D}\right|^{-2}$ for some smooth plurisubharmonic function $a$. Since $\theta_{X}+\sqrt{-1} \partial \bar{\partial} \varphi=$ $\sqrt{-1} \partial \bar{\partial}(a+\varphi)$ on this coordinate chart, it is enough to consider the following complex Monge-Ampère equation

$$
\operatorname{det}\left(u_{i, \bar{j}}\right)=f
$$


on an open subset $\Omega \Subset \mathbb{C}^{n} \backslash(D \cup F)$ by setting

$$
u=a+\varphi .
$$

It follows from our construction that we may assume that the function $f$ is a form of

$$
f=\left|w_{F}\right|^{-2 / l}\left|w_{D}\right|^{2 m / l} .
$$

Fix an unit vector $\zeta \in \mathbb{C}^{n}$. Differentiating the equation $\log \operatorname{det}\left(u_{i, \bar{j}}\right)=\log f$, we have

$$
u^{i, \bar{j}} u_{\zeta, \bar{\zeta}, i, \bar{j}}=(\log f)_{\zeta, \bar{\zeta}}+u^{i, \bar{l}} u^{k, \bar{j}} u_{\zeta, i, \bar{j}} u_{\bar{\zeta}, k, \bar{l}} \geq(\log f)_{\zeta, \bar{\zeta}}=0
$$

Here we have used the standard Einstein convention and the notation $\left(u^{i, \bar{j}}\right)=\left(\left(u_{i, \bar{j}}\right)^{t}\right)^{-1}$. Set

$$
a^{i, \bar{j}}=f u^{i, \bar{j}}
$$

Then, for any $i$, we can compute as follows:

$$
\left(a^{i, \bar{j}}\right)_{\bar{j}}=f f_{\bar{j}} u^{i, \bar{j}}-f u^{i, \bar{l}} u^{k, \bar{j}} u_{\bar{j}, k, \bar{l}}=f u^{k, \bar{l}} u_{\bar{j}, k, \bar{l}} u^{i, \bar{j}}-f u^{i, \bar{l}} u^{k, \bar{j}} u_{\bar{j}, k, \bar{l}}=0 .
$$

Thus, we obtain

$$
\left(a^{i, \bar{j}} u_{\zeta, \bar{\zeta}, i}\right)_{\bar{j}}=\left(a^{i, \bar{j}}\right)_{\bar{j}} u_{\zeta, \bar{\zeta}, i}+a^{i, \bar{j}} u_{\zeta, \bar{\zeta}, i, \bar{j}} \geq f(\log f)_{\zeta, \bar{\zeta}}=0
$$

Note that $u_{\zeta, \bar{\zeta}}$ is a subsolution of the equation $L v=0$, where $L v:=\sum_{i, j}\left(a^{i, \bar{j}} v_{i}\right)_{\bar{j}}$ and our construction implies that the operator $L$ is uniformly elliptic in the real sense. Then, we have the following estimate (see [8, Theorem 8.18]).

Lemma 24 The weak Harnack inequality

$$
r^{-2 n} \int_{B_{r}}\left(\sup _{B_{4 r}} u_{\zeta, \bar{\zeta}}-u_{\zeta, \bar{\zeta}}\right) \leq C_{H}\left(\sup _{B_{4 r}} u_{\zeta, \bar{\zeta}}-\sup _{B_{r}} u_{\zeta, \bar{\zeta}}\right),
$$

holds. Here, $B_{4 r}:=B\left(z_{0}, 4 r\right) \subset \Omega$ with $d\left(z_{0}, \partial \Omega\right)>4 r$. Moreover, in our case, we have the following estimate of the constant $C_{H}$ in Harnack inequality:

$$
C_{H}=O(\Lambda / \lambda) \text {. }
$$

Proof It suffices to show the estimate of the constant $C_{H}$. In our case, we will only consider the behavior of $\varphi$ in the neighborhood of $D \cup F$ and the $C^{2}$-estimate of $\varphi$ implies that

$$
\begin{aligned}
& u_{\zeta, \bar{\zeta}}=O\left(\left\|\sigma_{F}\right\|^{-2 / l}\right)=O(\Lambda) \\
& u_{\zeta, \bar{\zeta}}^{-1}=O\left(\left\|\sigma_{D}\right\|^{-2 m / l}\right)=O\left(\lambda^{-1}\right)
\end{aligned}
$$

as $\left\|\sigma_{F}\right\| \rightarrow 0$ and $\left\|\sigma_{D}\right\| \rightarrow 0$. Thus, the weak Harnack inequality implies that the lemma follows.

Remark 11 From the proof of [8, Theorem 8.18], we know that the optimal Harnack constant $C_{H}$ is estimated by

$$
C_{H}=C_{n}^{\sqrt{\Lambda / \lambda}}
$$

where $C_{n}$ depends only on $n$. 
For any $x, y \in B_{4 r}$, we obtain

$$
a^{i, \bar{j}}(y) u_{i, \bar{j}}(x)=f(y) u^{i, \bar{j}}(y) u_{i, \bar{j}}(x)=f(y) \operatorname{tr}\left(U(y)^{-1} U(x)\right) .
$$

Here, we set $U:=\left(u_{i, \bar{j}}\right)$. Clearly, $a^{i, \bar{j}}(y) u_{i, \bar{j}}(y)=n f(y)$. Since $\operatorname{det}\left(f(y)^{1 / n} U(y)^{-1}\right)=1$, we have

$$
\begin{aligned}
a^{i, \bar{j}}(y) u_{i, \bar{j}}(x) & =f(y)^{1-1 / n} \operatorname{tr}\left(f(y)^{1 / n} U(y)^{-1} U(x)\right) \\
& \geq n f(y)^{1-1 / n} \operatorname{det}(U(x))^{1 / n} \\
& =n f(y)^{1-1 / n} f(x)^{1 / n} .
\end{aligned}
$$

Here, we have used the following lemma (see [10, Lemma 5.8]):

Lemma 25 For any $A \in \mathscr{H}_{+}$, we have

$$
(\operatorname{det} A)^{1 / n}=\frac{1}{n} \inf \left\{\operatorname{tr}(A B) \mid B \in \mathscr{H}_{+}, \operatorname{det} B=1\right\} .
$$

Therefore, for any $x, y \in B_{4 r}$ and $\varepsilon \in(0,1)$, we have

$$
\begin{aligned}
a^{i, \bar{j}}(y)\left(u_{i, \bar{j}}(y)-u_{i, \bar{j}}(x)\right) & \leq n f(y)-n f(y)^{1-1 / n} f(x)^{1 / n} \\
& =n f(y)^{1-1 / n}\left(f(y)^{1 / n}-f(x)^{1 / n}\right) \\
& \leq C(\varepsilon)_{4}|x-y|^{\varepsilon},
\end{aligned}
$$

where

$$
C(\varepsilon)_{4}:=n \sup _{\Omega}\left(f^{1-1 / n}\right) \mathrm{Höl}_{\varepsilon, \Omega}\left(f^{1 / n}\right)
$$

and $\mathrm{Höl}_{\varepsilon, \Omega}$ denotes an $\varepsilon$-Hölder constant. In this case, the following estimates

$$
\begin{aligned}
\operatorname{Höl}_{\varepsilon, \Omega}\left(f^{1 / n}\right) & =O\left(\left\|\sigma_{F}\right\|^{-2 / n l-\varepsilon}\left\|\sigma_{D}\right\|^{2 m / n l-\varepsilon}\right) \\
\sup _{\Omega}\left(f^{1-1 / n}\right) & =O\left(\left\|\sigma_{F}\right\|^{-2(n-1) / n l}\left\|\sigma_{D}\right\|^{2 m(n-1) / n l}\right)
\end{aligned}
$$

implies that we have

$$
C(\varepsilon)_{4}=O\left(\left\|\sigma_{F}\right\|^{-2 / l-\varepsilon}\left\|\sigma_{D}\right\|^{2 m / l-\varepsilon}\right) .
$$

Remark 12 In [10, p. 375], they used the Lipscitz constant of $f$. But in our case, it is enough to use the Hölder constant of $f$ for sufficiently small $\varepsilon$.

Set $\lambda, \Lambda>0$ so that the eigenvalues of $\left(a^{i, \bar{j}}(y)\right)$ lie in the interval $[\lambda, \Lambda]$. Then, Lemma 23 implies that we can find unit vectors $\zeta_{1}, \ldots, \zeta_{N} \in \mathbb{C}^{n}$ such that for any $x, y \in \Omega$,

$$
a^{i, \bar{j}}(y)\left(u_{i, \bar{j}}(y)-u_{i, \bar{j}}(x)\right)=\sum_{k=1}^{N} \beta_{k}(y)\left(u_{\zeta_{k}, \bar{\zeta}_{k}}(y)-u_{\zeta_{k}, \bar{\zeta}_{k}}(x)\right),
$$

where $\beta_{k}(y) \in\left[\lambda_{*}, \Lambda_{*}\right]$ and $\lambda_{*}, \Lambda_{*}>0$.

Thus, we have

$$
\sum_{k=1}^{N} \beta_{k}(y)\left(u_{\zeta_{k}, \bar{\zeta}_{k}}(y)-u_{\zeta_{k}, \bar{\zeta}_{k}}(x)\right) \leq C(\varepsilon)_{4}|x-y|^{\varepsilon} .
$$


Set

$$
M_{k, r}:=\sup _{B_{r}} u_{\zeta_{k}, \bar{\zeta}_{k}}, \quad m_{k, r}:=\inf _{B_{r}} u_{\zeta_{k}, \bar{\zeta}_{k}}
$$

and

$$
\eta(r):=\sum_{k=1}^{N}\left(M_{k, r}-m_{k, r}\right)
$$

To obtain the Hölder condition

$$
\eta(r) \leq C r^{\tilde{\varepsilon}}
$$

for some $0<\tilde{\varepsilon}<1$, we need the following lemma from [8, p. 201, Lemma 8.23]:

Lemma 26 Let $\eta$ and $\sigma$ be non-decreasing functions defined on the interval $\left(0, R_{0}\right]$ such that there exist $\tau, \alpha \in(0,1)$ satisfying

$$
\eta(\tau r) \leq \alpha \eta(r)+\sigma(r)
$$

for all $r \in\left(0, R_{0}\right]$. Then, for any $\mu \in(0,1)$, we have

$$
\eta(R)<\frac{1}{\alpha}\left(\frac{R}{R_{0}}\right)^{(1-\mu)(\log \alpha / \log \tau)}+\frac{\sigma\left(R_{0}^{1-\mu} R^{\mu}\right)}{1-\alpha} .
$$

So, it suffices to show that

$$
\eta(r) \leq \delta \eta(4 r)+C r^{\varepsilon}, \quad 0<r<r_{0},
$$

where $\delta, \varepsilon \in(0,1)$ and $r_{0}>0$.

For fixed $k$, Harnack inequality before implies that

$$
\begin{aligned}
r^{-2 n} \int_{B_{r}} \sum_{l \neq k}\left(M_{l, 4 r}-u_{\zeta_{l}, \bar{\zeta}_{l}}\right) & =\sum_{l \neq k} r^{-2 n} \int_{B_{r}}\left(M_{l, 4 r}-u_{\zeta_{l}, \bar{\zeta}_{l}}\right) \\
& \leq \sum_{l \neq k} C_{H}\left(M_{l, 4 r}-M_{l, r}\right) \\
& \leq \sum_{l \neq k} C_{H}(\eta(4 r)-\eta(r)) \\
& =(N-1) C_{H}(\eta(4 r)-\eta(r)) .
\end{aligned}
$$

For $x \in B_{4 r}$ and $y \in B_{r}$, we have

$$
\begin{aligned}
\beta_{k}(y)\left(u_{\zeta_{k}, \bar{\zeta}_{k}}(y)-u_{\zeta_{k}, \bar{\zeta}_{k}}(x)\right) & \leq C(\varepsilon)_{4}|x-y|^{\varepsilon}+\sum_{l \neq k} \beta_{l}(y)\left(u_{\zeta_{l}, \bar{\zeta}_{l}}(x)-u_{\zeta_{l}, \bar{\zeta}_{l}}(y)\right) \\
& \leq 5 C(\varepsilon)_{4} r^{\varepsilon}+\Lambda_{*} \sum_{l \neq k}\left(M_{l, 4 r}-u_{\zeta_{l}, \bar{\zeta}_{l}}(y)\right) .
\end{aligned}
$$

Thus, for all $y \in B_{r}$, we have

$$
u_{\zeta_{k}, \bar{\zeta}_{k}}(y)-m_{k, 4 r} \leq \frac{1}{\lambda_{*}}\left(5 C(\varepsilon)_{4} r^{\varepsilon}+\Lambda_{*} \sum_{l \neq k}\left(M_{l, 4 r}-u_{\zeta_{l}, \bar{\zeta}_{l}}(y)\right)\right) .
$$


Therefore,

$$
\begin{aligned}
r^{-2 n} \int_{B_{r}}\left(u_{\zeta_{k}, \bar{\zeta}_{k}}(y)-m_{k, 4 r}\right) & \leq r^{-2 n} \int_{B_{r}} \frac{1}{\lambda_{*}}\left(5 C(\varepsilon)_{4} r^{\varepsilon}+\Lambda_{*} \sum_{l \neq k}\left(M_{l, 4 r}-u_{\zeta_{l}, \bar{\zeta}_{l}}(y)\right)\right) \\
& \leq \frac{5 C(\varepsilon)_{4}}{\lambda_{*}} r^{\varepsilon}+\frac{\Lambda_{*}}{\lambda_{*}} r^{-2 n} \int_{B_{r}} \sum_{l \neq k}\left(M_{l, 4 r}-u_{\zeta_{l}, \bar{\zeta}_{l}}\right) \\
& \leq \frac{5 C(\varepsilon)_{4}}{\lambda_{*}} r^{\varepsilon}+\frac{\Lambda_{*}}{\lambda_{*}}(N-1) C_{H}(\eta(4 r)-\eta(r)) .
\end{aligned}
$$

Using Harnack inequality again, we have

$$
\begin{aligned}
M_{k, 4 r}-m_{k, 4 r}= & r^{-2 n} \int_{B_{r}}\left(\sup _{B_{4 r}} u_{\zeta_{k}, \bar{\zeta}_{k}}-u_{\zeta_{k}, \bar{\zeta}_{k}}\right)+r^{-2 n} \int_{B_{r}}\left(u_{\zeta_{k}, \bar{\zeta}_{k}}(y)-m_{k, 4 r}\right) \\
\leq & C_{H}\left(M_{k, 4 r}-M_{k, r}\right)+\frac{5 C(\varepsilon)_{4}}{\lambda_{*}} r^{\varepsilon}+\frac{\Lambda_{*}}{\lambda_{*}}(N-1) C_{H}(\eta(4 r)-\eta(r)) \\
\leq & \left(C_{H}+\frac{\Lambda_{*}}{\lambda_{*}}(N-1) C_{H}\right) \eta(4 r) \\
& -\left(C_{H}+\frac{\Lambda_{*}}{\lambda_{*}}(N-1) C_{H}\right) \eta(r)+\frac{5 C(\varepsilon)_{4}}{\lambda_{*}} r^{\varepsilon} .
\end{aligned}
$$

Summing over $k$, we have

$$
\begin{aligned}
\eta(4 r) \leq & N\left(C_{H}+\frac{\Lambda_{*}}{\lambda_{*}}(N-1) C_{H}\right) \eta(4 r) \\
& -N\left(C_{H}+\frac{\Lambda_{*}}{\lambda_{*}}(N-1) C_{H}\right) \eta(r)+N \frac{5 C(\varepsilon)_{4}}{\lambda_{*}} r^{\varepsilon} .
\end{aligned}
$$

Thus, we obtain

$$
\eta(r) \leq \frac{N\left(C_{H}+\frac{\Lambda_{*}}{\lambda_{*}}(N-1) C_{H}\right)-1}{N\left(C_{H}+\frac{\Lambda_{*}}{\lambda_{*}}(N-1) C_{H}\right)} \eta(4 r)+\frac{\frac{5 C(\varepsilon)_{4}}{\lambda_{*}}}{C_{H}+\frac{\Lambda_{*}}{\lambda_{*}}(N-1) C_{H}} r^{\varepsilon} .
$$

Since we can take arbitrary $\lambda^{*} N<\lambda$ and $\Lambda^{*}>\Lambda$, we may assume that $\lambda^{*} N=\lambda$ and $\Lambda^{*}=\Lambda$. Thus, we have

Lemma 27 By taking $\varepsilon \leq 2 / l$, there exists $0<\tilde{\varepsilon}<\varepsilon$ with

$$
\|u\|_{C^{2, \tilde{\varepsilon}}}=O\left(\left(\frac{\Lambda}{\lambda}\right) C_{H}\right) .
$$

Proof In order to show this lemma, we apply Lemma 26 to the inequality (61). Set

$$
\alpha:=\frac{N\left(C_{H}+\frac{\Lambda}{\lambda}(N-1) C_{H}\right)-1}{N\left(C_{H}+\frac{\Lambda}{\lambda}(N-1) C_{H}\right)},
$$

where this is the coefficient of $\eta(4 r)$ in (61). Then, we have the following estimates:

$$
\frac{1}{\alpha}=O(1), \quad \frac{1}{1-\alpha}=O\left((\Lambda / \lambda) C_{H}\right)
$$


Here, we have used the fact that the number $N$ depends only on the dimension $n$ (Remark 10). Define a non-decreasing function $\sigma$ by

$$
\sigma(r):=\frac{\frac{5 C(\varepsilon)_{4}}{\lambda}}{C_{H}+\frac{\Lambda}{\lambda}(N-1) C_{H}} r^{\varepsilon} .
$$

Here, this is the second term in the right hand side of the inequality (61). Recall the estimate (60)

$$
C(\varepsilon)_{4}=O\left(\left\|\sigma_{F}\right\|^{-2 / l-\varepsilon}\left\|\sigma_{D}\right\|^{2 m / l-\varepsilon}\right)
$$

and Lemma 24. The assumption that $\varepsilon \leq 2 / l$ implies that we have the following

$$
\frac{\frac{5 C(\varepsilon)_{4}}{\lambda}}{C_{H}+\frac{\Lambda}{\lambda}(N-1) C_{H}}=O(1) .
$$

Lemma 26 implies that we have

$$
\eta(r)<\frac{1}{\alpha}\left(\frac{r}{r_{0}}\right)^{(1-\mu)(\log \alpha / \log (1 / 4))}+\frac{\sigma\left(r_{0}^{1-\mu} r^{\mu}\right)}{1-\alpha},
$$

for any $\mu \in(0,1)$. Take $\mu \in(0,1)$ so that

$$
(1-\mu)(\log \alpha / \log (1 / 4))>\mu \varepsilon .
$$

Thus, we have

$$
\eta(r)<O\left((\Lambda / \lambda) C_{H}\right) \sigma\left(r_{0}^{1-\mu} r^{\mu}\right)
$$

Set $\tilde{\varepsilon}:=\varepsilon \mu<\varepsilon$. From the interior Hölder estimate for solutions of Poisson's equation [8, Theorem 4.6], we finish the proof.

Recall the relation (57) between $u$ and $\varphi$. Lemma 24 implies

Proposition 11 For the domain $\Omega \Subset X \backslash(D \cup F)$, we have

$$
\|\varphi\|_{C^{2, \tilde{\varepsilon}(\Omega)}}=O\left(\left(\left\|\sigma_{D}\right\|^{-2 m / l}\left\|\sigma_{F}\right\|^{-2 / l}\right)^{2}\right)
$$

as $\sigma_{D}, \sigma_{F} \rightarrow 0$.

\subsection{The third and the forth order estimates}

In this subsection, we prove Theorem 3. This subsection also follows from [10, Chapter 14]. To consider higher order estimates, we recall the Schauder estimate with respect to the elliptic linear operator defined by the Kähler metric $\theta_{X}+\sqrt{-1} \partial \bar{\partial} \varphi$. Note that the complex MongeAmpère operator defined by $F\left(D^{2} u\right)=\operatorname{det}\left(u_{i, \bar{j}}\right)$ is elliptic if the $2 n \times 2 n$ real symmetric matrix $A:=\left(\partial F / \partial u_{p, q}\right)$ is positive. Here, $u_{p, q}$ is the element of the real Hessian $D^{2} u$. We can show that this real symmetric matrix $A$ is given by $\left.\frac{d}{d t} F\left(D^{2} u+t B\right)\right|_{t=0}=\operatorname{tr}\left(A^{t} B\right)$. From [4] (see also [10, Exercise 14.8]), we have

Lemma 28 One has

$$
\lambda_{\min }\left(\partial F / \partial u_{p, q}\right)=\frac{\operatorname{det}\left(u_{i, \bar{j}}\right)}{4 \lambda_{\max }\left(u_{i, \bar{j}}\right)}, \quad \lambda_{\max }\left(\partial F / \partial u_{p, q}\right)=\frac{\operatorname{det}\left(u_{i, \bar{j}}\right)}{4 \lambda_{\min }\left(u_{i, \bar{j}}\right)},
$$


where $\lambda_{\min }\left(\partial F / \partial u_{p, q}\right)$ and $\lambda_{\max }\left(\partial F / \partial u_{p, q}\right)$ denote minimal and maximal eigenvalue of the matrix $\left.\left(\partial F / \partial u_{p, q}\right)\right)_{p . q}$ respectively.

Then, we can estimate the ellipticity in the real sense. We can apply the standard elliptic theory to the following equation

$$
F\left(D^{2} u\right)=f=\left|w_{F}\right|^{-2 / l}\left|w_{D}\right|^{2 m / l} .
$$

For a fixed vector $\zeta \in \mathbb{C}^{n}$ and sufficiently small $h>0$, we define functions by

$$
u^{h}(x):=\frac{u(x+h \zeta)-u(x)}{h}
$$

and

$$
a_{h}^{p, q}(x):=\int_{0}^{1} \frac{\partial F}{\partial u_{p, q}}\left(t D^{2} u(x+h \zeta)+(1-t) D^{2} u(x)\right) d t .
$$

Thus, we have the following:

$$
a_{h}^{p, q}(x) u_{p, q}^{h}(x)=\frac{1}{h} \int_{0}^{1} \frac{d}{d t} F\left(t D^{2} u(x+h \zeta)+(1-t) D^{2} u(x)\right) d t=f^{h}(x) .
$$

From the definition of $a_{h}^{p, q}$, we obtain

$$
\left\|a_{h}^{p, q}\right\|_{C^{0, \tilde{\varepsilon}}} \leq C\|u\|_{C^{2, \tilde{\varepsilon}}}^{n-1}=O\left((\Lambda / \lambda)^{2(n-1)}\right)
$$

for sufficiently small $h>0$.

The Schauder estimate implies

Proposition 12 There exists $C_{S}>0$ such that

$$
\left\|u^{h}\right\|_{C^{2, \tilde{\varepsilon}}} \leq C_{S}\left(\left\|f^{h}\right\|_{C^{0, \tilde{\varepsilon}}}+\left\|u^{h}\right\|_{C^{0}}\right)
$$

for any $h>0$.

Therefore, we can obtain the estimate of derivatives of the solution $\varphi$ in the desired direction by taking a suitable vector $\zeta$ and $h \rightarrow 0$. The constant $C_{S}$ in Proposition 12 also depends on the maximal ratio of the eigenvalues $\Lambda / \lambda$ and the dimension $n$. By examining the proof of [8, Lemma 6.1 and Theorem 6.2], there is a positive constant $s(n)$ depending only on the dimension $n$ such that

$$
C_{S}=O\left((\Lambda / \lambda)^{s(n)}\right) .
$$

As $h \rightarrow 0$, we have the following third order estimates of $\varphi$ :

Proposition 13 For any multi-index $\alpha=\left(\alpha_{1}, \ldots, \alpha_{n}\right)$ satisfying $\sum_{i} \alpha_{i}=2$, we have

$$
\begin{aligned}
& \left|\frac{\partial}{\partial z^{i}} \partial^{\alpha} \varphi\right|=O\left(C_{S}\left|w_{D}\right|^{-4 m / l}\left|w_{F}\right|^{-4 / l}\right), \\
& \left|\frac{\partial}{\partial w_{F}} \partial^{\alpha} \varphi\right|=O\left(C_{S}\left|w_{D}\right|^{-4 m / l}\left|w_{F}\right|^{-1-4 / l}\right), \\
& \left|\frac{\partial}{\partial w_{D}} \partial^{\alpha} \varphi\right|=O\left(C_{S}\left|w_{D}\right|^{-1-4 m / l}\left|w_{F}\right|^{-4 / l}\right),
\end{aligned}
$$

as $\left|w_{D}\right|,\left|w_{F}\right| \rightarrow 0$.

From the discussion above, we can prove Theorem 3. 
Proof of Theorem 3 Let $\dot{a}_{h}^{p, q}$ be a differential of $a_{h}^{p, q}$ in some direction. From the definition of $a_{h}^{p, q}$, we know that

$$
\left\|\dot{a}_{h}^{p, q}\right\|_{C^{0, \tilde{\varepsilon}}} \leq C\|\dot{u}\|_{C^{2, \tilde{\varepsilon}}}\|u\|_{C^{2, \tilde{\varepsilon}}}^{n-2} .
$$

Thus, by differentiating the equation $a_{h}^{p, q}(x) u_{p, q}^{h}(x)=f^{h}(x)$, Schauder estimate implies again the following inequality:

$$
\left\|\dot{u}^{h}\right\|_{C^{2, \tilde{\varepsilon}}} \leq C_{S}\left(\left\|\dot{f}^{h}-\dot{a}_{h}^{p, q} u_{p, q}^{h}\right\|_{C^{0, \tilde{\varepsilon}}}+\left\|\dot{u}^{h}\right\|_{C^{0}}\right) .
$$

Thus, we finish the proof pf Theorem 3 by taking a suitable vector $\zeta$ and $h \rightarrow 0$.

Remark 13 By examining the proof of [8, Lemma 6.1 and Theorem 6.2] and the discussion above, we can find that

$$
a=a(n)=O\left(n^{2}\right) .
$$

\section{Proof of Theorem 4}

In this section, we prove Theorem 4. To compute the scalar curvature of the Kähler metric $\omega_{c, v, \eta}$, we have to consider the inverse matrix (Lemma 5). Since we assume that the divisor $D+F$ is simple normal crossing, we can choose block matrices in suitable directions in local holomorphic coordinates defining hypersurfaces $D$ and $F$. To prove Theorem 4 , we consider the case that the parameter $\eta=\left(\eta_{1}, \eta_{2}, \eta_{3}\right)$ depends on $c>0$. More precisely, we set $\eta_{i}:=a_{i} c$ for $i=1,2$ for $a_{i} \in(0,1)$ and $\eta_{3}$ a fixed positive real number. We use many parameters, i.e., $c, v, \beta, \kappa, \eta, a_{i}$. When we want to make the scalar curvature $S\left(\omega_{c, v, \eta}\right)$ small, we take sufficiently large $c$ and sufficiently small $v$. On the other hand, we don't make other parameters $\beta, \kappa, a_{i}$ close to $\infty, 0$ or 1 . Namely, the parameters $\beta, \kappa, a_{i}$ are bounded in this sense. Settings of these bounded parameters will be given later.

Proof of Theorem 4 Take a relatively compact domain $Y \Subset X \backslash(D \cup F)$. Recall that the function $G_{v}^{\beta}(\beta b)$ is defined by

$$
G_{v}^{\beta}(\beta b):=\int_{b_{0}}^{\beta b}\left(\frac{1}{e^{-y}+v}\right)^{1 / \beta} d y .
$$

Immediately, we have $G_{v}^{\beta}(\beta b)<\beta e^{b}$ and $G_{v}^{\beta}(\beta b) \rightarrow \beta e^{b}$ as $v \rightarrow 0$. So, we can find a sufficiently large number $c_{0}=c_{0}(Y)>0$ so that

$$
Y \Subset\left\{t+\varphi+c_{0}>\max \left\{\Theta(t), \tilde{G}_{v}^{\beta}(b)\right\}\right\} \Subset X \backslash(D \cup F)
$$

for any $v>0$. Here, $\tilde{G}_{v}^{\beta}(b)=G_{v}^{\beta}(\beta b)+\kappa \Theta(t)$. For simplicity, we write $\varphi+c_{0}$ by the same symbol $\varphi$.

Recall that the property d) of the regularized maximum in Lemma 20. If the following inequality

$$
\max _{j \neq k}\left\{t_{j}+\eta_{j}\right\}<t_{k}-\eta_{k}
$$

holds for some $k$, we have $M_{\eta}(t)=t_{k}$. For instance, in our case, if we consider the region defined by the following inequality

$$
\max \left\{\tilde{G}_{v}^{\beta}(\beta b)+\eta_{2}, t+\varphi+c+\eta_{3}\right\}<\Theta(t)-\eta_{1},
$$


we have $M_{c, v, \eta}=\Theta(t)$. Note that this region is contained in a sufficiently small neighborhood of $D$. In this case, we don't have to estimate the scalar curvature $S\left(\omega_{c, v, \eta}\right)$ since $S\left(\omega_{c, v, \eta}\right)=$ $S\left(\omega_{0}\right)$ on this region and the estimate of $S\left(\omega_{0}\right)$ have been obtained in Lemma 50 before. Similarly, if the value of $M_{c, v, \eta}$ corresponds to one of the other variables $\tilde{G}_{v}^{\beta}(b), t+\varphi+c$, Lemma 22 and the Ricci-flatness of the Kähler metric $\sqrt{-1} \partial \bar{\partial}(t+\varphi)$ implies that $S\left(\omega_{c, v, \eta}\right)$ is under control on such regions. Thus, it suffices for us to study the $S\left(\omega_{c, v, \eta}\right)$ on the other regions defined by the inequalities

$$
\begin{aligned}
t_{k}+\eta_{k} & <\max _{j \neq k}\left\{t_{j}-\eta_{j}\right\}, \\
\left|t_{i}-t_{j}\right| & <\eta_{i}+\eta_{j},
\end{aligned}
$$

for $i, j \neq k$ and

$$
\begin{aligned}
& \left|t_{1}-t_{2}\right|<\eta_{1}+\eta_{2}, \\
& \left|t_{2}-t_{3}\right|<\eta_{2}+\eta_{3}, \\
& \left|t_{1}-t_{3}\right|<\eta_{1}+\eta_{3} .
\end{aligned}
$$

So we have to study $S\left(\omega_{c, v, \eta}\right)$ on four regions defined by the inequalities above.

Directly, we have

$$
\begin{aligned}
\omega_{c, v, \eta}= & \sqrt{-1} g_{i, \bar{j}} d z^{i} \wedge d \bar{z}^{j} \\
= & \frac{\partial M_{c, v, \eta}}{\partial t_{1}} \omega_{0}+\frac{\partial M_{c, v, \eta}}{\partial t_{2}}\left(\gamma_{v}^{\beta}+\kappa \omega_{0}\right)+\frac{\partial M_{c, v, \eta}}{\partial t_{3}} \sqrt{-1} \partial \bar{\partial}(t+\varphi) \\
& +\left[\partial \Theta(t) \partial \tilde{G}_{v}^{\beta}(b) \partial(t+\varphi)\right]\left[\frac{\partial^{2} M_{c, v, \eta}}{\partial t_{i} \partial t_{j}}\right]\left[\bar{\partial} \Theta(t) \bar{\partial} \tilde{G}_{v}^{\beta}(b) \bar{\partial}(t+\varphi)\right]^{t} .
\end{aligned}
$$

It follows from the convexity of $M_{\eta}$ that the last term is semi-positive. When we compute the scalar curvature of $\omega_{c, v, \eta}$, the difficulty comes from terms $\partial \Theta(t) \wedge \bar{\partial} \Theta(t)$ and $\partial \tilde{G}_{v}^{\beta}(b) \wedge \bar{\partial} \tilde{G}_{v}^{\beta}(b)$. For these terms, since functions $t$ and $b$ are defined by Hermitian norms of holomorphic sections, it suffices to focus on derivatives in normal directions of smooth hypersurfaces $D$ and $F$ by taking suitable local trivializations of line bundles $L_{X}$ and $K_{X}^{-l} \otimes L_{X}^{m}$ respectively. The reason why scalar curvatures of two Kähler metrics $\omega_{0}, \gamma_{v}^{\beta}$ are under control near these hypersurfaces $D, F$ is that Ricci curvatures are bounded and Kähler metrics grow asymptotically near these hypersurfaces. Thus, it suffices for us to focus on derivatives of $\varphi$ and $M_{\eta}$ arising in Ricci tensors. The higher order derivatives of $\varphi$ are estimated in the previous section (Theorem 3$)$. In addition, the definition of a parameter $\eta=\left(\eta_{i}\right)=\left(a_{1} c, a_{2} c, \eta_{3}\right)$ and Lemma 21 imply that the higher order derivatives in the first or the second variable of $M_{\eta}$ are estimated by some negative power of $c>0$. To estimate $S\left(\omega_{c, v, \eta}\right)$ on each region, we divide the proof of Theorem 4 into the following four claims.

Claim On the region defined by

$$
\begin{aligned}
(t+\varphi+c)+\eta_{3} & <\max \left\{\Theta(t)-\eta_{1}, \tilde{G}_{v}^{\beta}(b)-\eta_{2}\right\}, \\
\left|\Theta(t)-\tilde{G}_{v}^{\beta}(b)\right| & <\eta_{1}+\eta_{2},
\end{aligned}
$$

we can make the scalar curvature $S\left(\omega_{c, v}\right)$ small arbitrarily by taking a sufficiently large $c$.

Proof On this region, we can write as

$$
\omega_{c, v, \eta}=\frac{\partial M_{c, v, \eta}}{\partial t_{1}} \omega_{0}+\frac{\partial M_{c, v, \eta}}{\partial t_{2}}\left(\gamma_{v}^{\beta}+\kappa \omega_{0}\right)
$$




$$
+\left[\partial \Theta(t) \partial \tilde{G}_{v}^{\beta}(b)\right]\left[\frac{\partial^{2} M_{c, v, \eta}}{\partial t_{i} \partial t_{j}}\right]\left[\bar{\partial} \Theta(t) \bar{\partial} \tilde{G}_{v}^{\beta}(b)\right]^{t} .
$$

To prove this claim, we need the following lemma.

Lemma 29 Take a point $p \in D \cap F$ and local holomorphic coordinates $\left(z^{1}, \ldots, z^{n-2}, w_{F}\right.$, $w_{D}$ ) centered at $p$ satisfying $D=\left\{w_{D}=0\right\}$ and $F=\left\{w_{F}=0\right\}$. By taking suitable local trivializations of $L_{X}$ and $K_{X}^{-l} \otimes L_{X}^{m}$, we may assume that if $\left(z^{1}, \ldots, z^{n-2}, w_{F}, w_{D}\right)=$ $\left(0, \ldots, 0, w_{F}, w_{D}\right)$, we have

$$
\begin{aligned}
\partial \Theta(t) \wedge \bar{\partial} \Theta(t)= & O\left(\left|w_{F}\right|^{2}\left|w_{D}\right|^{-4 \hat{S}_{D} / n(n-1)}\right) d w_{F} \wedge d \overline{w_{F}} \\
& +O\left(\left|w_{F}\right|\left|w_{D}\right|^{-1-4 \hat{S}_{D} / n(n-1)}\right)\left(d w_{D} \wedge d \overline{w_{F}}+d w_{F} \wedge d \overline{w_{D}}\right) \\
& +O\left(\left|w_{D}\right|^{-2-4 \hat{S}_{D} / n(n-1)}\right) d w_{D} \wedge d \overline{w_{D}}, \\
\partial G_{v}^{\beta}(\beta b) \wedge \bar{\partial} G_{v}^{\beta}(\beta b)= & O\left(\left(\left|w_{F}\right|^{2 \beta}+v\right)^{-2 / \beta}\left|w_{F}\right|^{-2}\right) d w_{F} \wedge d \overline{w_{F}} \\
& +O\left(\left|w_{F}\right|^{-1}\left|w_{D}\right|\left(\left|w_{F}\right|^{2 \beta}+v\right)^{-2 / \beta}\right)\left(d w_{D} \wedge d \overline{w_{F}}+d w_{F} \wedge d \overline{w_{D}}\right) \\
& +O\left(\left(\left|w_{F}\right|^{2 \beta}+v\right)^{-2 / \beta}\left|w_{D}\right|^{2}\right) d w_{D} \wedge d \overline{w_{D}} .
\end{aligned}
$$

From the definition of this region, we obtain

$$
\omega_{c, v, \eta}=\left[\begin{array}{ccccc}
g_{1, \overline{1}} & \cdots & g_{1, \overline{n-2}} & g_{1, \overline{n-1}} & g_{1, \bar{n}} \\
\vdots & \ddots & \vdots & \vdots & \vdots \\
g_{n-2, \overline{1}} & \cdots & g_{n-2, \overline{n-2}} & g_{n-2, \overline{n-1}} & g_{n-2, \bar{n}} \\
g_{n-1, \overline{1}} & \cdots & g_{n-1, \overline{n-2}} & \left(\left|w_{F}\right|^{2 \beta}+v\right)^{-2 / \beta}\left|w_{F}\right|^{-2} & \left|w_{F}\right|^{-1}\left|w_{D}\right|\left(\left|w_{F}\right|^{2 \beta}+v\right)^{-2 / \beta} \\
g_{n, \overline{1}} & \cdots & g_{n, \overline{n-2}} & \left|w_{F}\right|^{-1}\left|w_{D}\right|\left(\left|w_{F}\right|^{2 \beta}+v\right)^{-2 / \beta} & \left|w_{D}\right|^{-2-4 \hat{S}_{D} / n(n-1)}
\end{array}\right]
$$

as $w_{D}, w_{F} \rightarrow 0$.

In particular, coefficients $g_{i, \bar{j}}$ for $1 \leq i, j \leq n-2$ come from Kähler metrics $\omega_{0}$ and $\gamma_{v}^{\beta}$. Thus,

$$
\left[\begin{array}{ccc}
g_{1, \overline{1}} & \cdots & g_{1, \overline{n-2}} \\
\vdots & \ddots & \vdots \\
g_{n-2, \overline{1}} & \cdots & g_{n-2, \overline{n-2}}
\end{array}\right]=O\left(\left|w_{D}\right|^{-2 \hat{S}_{D} / n(n-1)}+\left(\left|w_{F}\right|^{2 \beta}+v\right)^{-1 / \beta}\right) .
$$

For other blocks, we similarly have

$$
\left[\begin{array}{cc}
g_{1, \overline{n-1}} & g_{1, \bar{n}} \\
\vdots & \vdots \\
g_{n-2, \overline{n-1}} & g_{n-2, \bar{n}}
\end{array}\right]=O\left(\left|w_{D}\right|^{-2 \hat{S}_{D} / n(n-1)}+\left(\left|w_{F}\right|^{2 \beta}+v\right)^{-1 / \beta}\right) .
$$

From Lemma 5, we have

$$
g^{i, \bar{j}}=\left[\begin{array}{ccccc}
g^{1, \overline{1}} & \cdots & g^{1, \overline{n-2}} & g^{1, \overline{n-1}} & g^{1, \bar{n}} \\
\vdots & \ddots & \vdots & \vdots & \vdots \\
g^{n-2, \overline{1}} & \cdots & g^{n-2, \overline{n-2}} & g^{n-2, \overline{n-1}} & g^{n-2, \bar{n}} \\
g^{n-1, \overline{1}} & \cdots & g^{n-1, \overline{n-2}} & c\left(\left|w_{F}\right|^{2 \beta}+v\right)^{2 / \beta}\left|w_{F}\right|^{2} & c\left|w_{D}\right|^{3+4 \hat{S}_{D} / n(n-1)}\left|w_{F}\right| \\
g^{n, \overline{1}} & \cdots & g^{n, \overline{n-2}} & c\left|w_{D}\right|^{3+4 \hat{S}_{D} / n(n-1)}\left|w_{F}\right| & c\left|w_{D}\right|^{2+4 \hat{S}_{D} / n(n-1)}
\end{array}\right]
$$

as $w_{D}, w_{F} \rightarrow 0$. Since metric tensors $g^{i, \bar{j}}$ with $i, j \neq n-1, n$ come from Kähler metrics $\omega_{0}$ and $\gamma_{v}^{\beta}$ whose scalar curvature have been already known. Thus, it is enough to study the 
case that $i=n-1, n$ and $j=n-1, n$. Recall that the components of the Ricci tensor are defined by $R_{i, \bar{j}}:=-g^{p, \bar{q}} \partial^{2} g_{p, \bar{q}} / \partial z^{i} \partial \bar{z}^{j}+g^{k, \bar{q}} g^{p, \bar{l}}\left(\partial g_{k, \bar{l}} / \partial z^{i}\right)\left(\partial g_{p, \bar{q}} / \partial \bar{z}^{j}\right)$. So, the Ricci form $\operatorname{Ric}\left(\omega_{c, v, \eta}\right)$ is written as

$$
\left[\begin{array}{ccccc}
R_{1, \overline{1}} & \cdots & R_{1, \overline{n-2}} & R_{1, \overline{n-1}} & R_{1, \bar{n}} \\
\vdots & \ddots & \vdots & \vdots & \vdots \\
R_{n-2, \overline{1}} & \cdots & R_{n-2, \overline{n-2}} & R_{n-2, \overline{n-1}} & R_{n-2, \bar{n}} \\
R_{n-1, \overline{1}} & \cdots & R_{n-1, \overline{n-2}} & c^{-3}\left(\left|w_{F}\right|^{2 \beta}+v\right)^{-2 / \beta}\left|w_{F}\right|^{-2} & c^{-3}\left|w_{F}\right|^{-1}\left|w_{D}\right|\left(\left|w_{F}\right|^{2 \beta}+v\right)^{-2 / \beta} \\
R_{n, \overline{1}} & \cdots & R_{n, \overline{n-2}} & c^{-3}\left|w_{F}\right|^{-1}\left|w_{D}\right|\left(\left|w_{F}\right|^{2 \beta}+v\right)^{-2 / \beta} & c^{-3}\left|w_{D}\right|^{-2-4 \hat{S}_{D} / n(n-1)}
\end{array}\right]
$$

as $w_{D}, w_{F} \rightarrow 0$ and the other components of the Ricci tensor $R_{i, \bar{j}}$ for $1 \leq i \leq n-2$ are under control.

By taking the trace, we obtain the following:

$$
S\left(\omega_{c, v, \eta}\right)=O\left(c^{-2}\right) .
$$

Remark 14 On the region in the previous claim, there are the terms $\partial \Theta(t) \wedge \bar{\partial} \Theta(t)$ and $\partial G_{v}^{\beta}(\beta b) \wedge \bar{\partial} G_{v}^{\beta}(\beta b)$ in the complete Kähler metric $\omega_{c, v, \eta}$. Thus, $\left(X \backslash D, \omega_{c, v, \eta}\right)$ is not of asymptotically conical geometry and we can't use the analysis in Sect. 5 with respect to this Kähler metric $\omega_{c, v, \eta}$. This problem will be solved in the next section.

We proceed to the estimate of $S\left(\omega_{c, v, \eta}\right)$ on another region.

Claim Consider the region defined by

$$
\begin{aligned}
\tilde{G}_{v}^{\beta}(b)+\eta_{2} & <\max \left\{\Theta(t)-\eta_{1},(t+\varphi+c)-\eta_{3}\right\}, \\
|\Theta(t)-(t+\varphi+c)| & <\eta_{1}+\eta_{3} .
\end{aligned}
$$

Take parameters $\eta, \kappa$ so that

$$
(1-\kappa) c+\kappa \eta_{1}-\eta_{2}=\left(1-\kappa+\kappa a_{1}-a_{2}\right) c=0
$$

for any $c>0$. Then, we can make the scalar curvature $S\left(\omega_{c, v}\right)$ small arbitrarily by taking a sufficiently large $c$.

Proof On this region, since

$$
M_{c, v, \eta}=M_{\eta}(\Theta(t), t+\varphi+c)
$$

from Lemma 20, we have

$$
\begin{aligned}
\omega_{c, v, \eta}= & \frac{\partial M_{c, v, \eta}}{\partial t_{1}} \omega_{0}+\frac{\partial M_{c, v, \eta}}{\partial t_{3}} \sqrt{-1} \partial \bar{\partial}(t+\varphi) \\
& +[\partial \Theta(t) \partial(t+\varphi)]\left[\frac{\partial^{2} M_{c, v, \eta}}{\partial t_{i} \partial t_{j}}\right][\bar{\partial} \Theta(t) \bar{\partial}(t+\varphi)]^{t} .
\end{aligned}
$$

From the hypothesis of this claim, we have

$$
\begin{aligned}
G_{v}^{\beta}(\beta b) & <(t+\varphi+c)+\eta_{3}-\kappa \Theta(t)-\eta_{2} \\
& <(1-\kappa)(t+\varphi+c)+\kappa\left(\eta_{1}+\eta_{3}\right)+\eta_{3}-\eta_{2} \\
& =(1-\kappa)(t+\varphi)+(1+\kappa) \eta_{3} .
\end{aligned}
$$


By taking a small $v>0$ and a suitable $b_{0}$ in the definition of the function $G_{v}^{\beta}(\beta b)$, we may assume that

$$
\beta b<G_{v}^{\beta}(\beta b) .
$$

From a priori estimate due to Kołodziej [13] again, $\varphi$ is bounded on $X$. So, on this region, we have the following inequality:

$$
\left\|\sigma_{F}\right\|^{-2 \beta /(1-\kappa)}<C\left\|\sigma_{D}\right\|^{-2}
$$

for some constant $C>0$ depending only on the $C^{0}$-norm of $\varphi$. By taking $\kappa$ close to 1 which depends on $m, l$ and $a=a(n)$ in Theorem 3, we may assume that

$$
\left\|\sigma_{F}\right\|^{-2-2 a / l}<C\left\|\sigma_{D}\right\|^{-2 a m / l} .
$$

Thus, on this region, the growth of derivatives of $\varphi$ can be controlled by the Kähler metric $\omega_{0}$. Take a point in $D \backslash(D \cap F)$ and local holomorphic coordinates $\left(z^{i}\right)_{i=1}^{n}=\left(z^{1}, \ldots, z^{n-1}, w_{D}\right)$ satisfying $D=\left\{w_{D}=0\right\}$. Then, we have

$$
\left|\frac{\partial^{2}}{\partial z^{i} \partial \bar{z}^{j}} \partial^{\alpha} \varphi\right|=O\left(\left|w_{D}\right|^{-2 a m / l}\right),
$$

if $1 \leq i, j \leq n-1$ and

$$
\left|\frac{\partial^{2}}{\partial w_{D} \partial \overline{w_{D}}} \partial^{\alpha} \varphi\right|=O\left(\left|w_{D}\right|^{-2-2 a m / l}\right) .
$$

Similarly, we have

Lemma 30 By taking a suitable local holomorphic trivialization of $L_{X}$, we may assume that if $\left(z^{1}, \ldots, z^{n-1}, w_{D}\right)=\left(0, \ldots, 0, w_{D}\right)$, we have

$$
\partial \Theta(t) \wedge \bar{\partial} \Theta(t)=O\left(\left|w_{D}\right|^{-2-4 \hat{S}_{D} / n(n-1)}\right) d w_{D} \wedge d \overline{w_{D}} .
$$

Recall the hypothesis

$$
\frac{a m}{2 l}<\frac{\hat{S}_{D}}{n(n-1)} .
$$

So, Theorem 3 implies that the growth of the Kähler metric $\omega_{c, v, \eta}$ is greater then the growth of the higher order derivatives of $\varphi$. Thus, Lemma 5 show that higher order derivatives including $\partial^{4} \varphi / \partial w^{2} \partial \bar{w}^{2}$ are controlled by taking the trace with respect to $\omega_{c, v, \eta}$. Therefore, we can ignore derivatives of $\varphi$ arising in the components of the Ricci tensor and we have

$$
S\left(\omega_{c, v, \eta}\right)=O\left(c^{-2}\right) .
$$

We proceed to the estimate of $S\left(\omega_{c, v, \eta}\right)$ the following region.

Claim Consider the region defined by

$$
\begin{aligned}
\Theta(t)+\eta_{1} & <\max \left\{\tilde{G}_{v}^{\beta}(b)-\eta_{2},(t+\varphi+c)-\eta_{3}\right\}, \\
\left|\tilde{G}_{v}^{\beta}(b)-(t+\varphi+c)\right| & <\eta_{2}+\eta_{3} .
\end{aligned}
$$

By choosing sufficiently small number $v>0$ so that

$$
\left(\left\|\sigma_{F}\right\|^{2 \beta}+v\right)^{2 / \beta}<\left\|\sigma_{F}\right\|^{4 a m / l}
$$

holds on this region, we can make the scalar curvature $S\left(\omega_{c, v}\right)$ small arbitrarily by taking a sufficiently large $c$. 
Proof The reason why we can find a sufficiently small number $v>0$ satisfying the statement in this claim is that $\min \left\{\left\|\sigma_{F}\right\|\right\}$ on this region increase as $v \rightarrow 0$ and $4 a m / l<4$. In order to prove this Claim, we need the following lemma.

Lemma 31 By taking a suitable local trivialization of $K_{X}^{-l} \otimes L_{X}^{m}$, we may assume that if $\left(z^{1}, \ldots, z^{n-2}, w_{F}, z_{n}\right)=\left(0, \ldots, 0, w_{F}, 0\right)$, we have

$$
\partial G_{v}^{\beta}(\beta b) \wedge \bar{\partial} G_{v}^{\beta}(\beta b)=O\left(\left(\left|w_{F}\right|^{2 \beta}+v\right)^{-2 / \beta}\left|w_{F}\right|^{-2}\right) d w_{F} \wedge d \overline{w_{F}} .
$$

Thus, we can prove this claim by using the same way in the previous claim.

The remained case is the following claim.

Claim On the region defined by

$$
\begin{aligned}
\left|\Theta(t)-\tilde{G}_{v}^{\beta}(b)\right| & <\eta_{1}+\eta_{2}, \\
\left|\tilde{G}_{v}^{\beta}(b)-(t+\varphi+c)\right| & <\eta_{2}+\eta_{3}, \\
|\Theta(t)-(t+\varphi+c)| & <\eta_{1}+\eta_{3},
\end{aligned}
$$

we can make the scalar curvature $S\left(\omega_{c, v, \eta}\right)$ small arbitrarily by taking a sufficiently large $c$.

Proof On this region, we can show that $S\left(\omega_{c, v, \eta}\right)=O\left(c^{-2}\right)$ similarly. Thus, we have finished proving Theorem 4.

\section{Proof of Theorem 5}

In this section, we prove Theorem 5. We construct the complete Kähler metric $\omega_{F}=\omega_{c, v, \eta}$ whose scalar curvature is arbitrarily small on $X \backslash D$ in previous sections. For $\beta \in \mathbb{Z}_{>0}$, take a holomorphic section $\sigma_{0} \in H^{0}\left(K_{X}^{-l} \otimes L_{X}^{m+\beta}\right)$. We may assume that $D+F_{0}$ is simple normal crossing, where $F_{0}$ is a smooth hypersurface defined by $\sigma_{0}$. Let $\left(\sigma_{i}\right)_{i} \subset H^{0}\left(K_{X}^{-l} \otimes L_{X}^{m+\beta}\right)$ be an orthonormal basis with respect to the $L^{2}$ inner product. Take a sufficiently small number $\tau \in \mathbb{R}$. Write $h(l, m, \beta):=\operatorname{dim} H^{0}\left(K_{X}^{-l} \otimes L_{X}^{m+\beta}\right)$. For $s=\left(s_{i}\right)_{i} \in \mathbb{D}^{h(l, m, \beta)}:=\{z=$ $\left.\left(z_{i}\right) \in \mathbb{C}^{h(l, m, \beta)}|| z_{i} \mid \leq 1\right\}$, define a meromorphic section of the line bundle $K_{X}^{-l} \otimes L_{X}^{m}$ by

$$
\sigma_{s}:=\left(\sigma_{0}+\tau \sum_{i=1}^{h(l, m, \beta)} s_{i} \sigma_{i}\right) \otimes \sigma_{D}^{-\beta}
$$

Note that by taking a sufficiently small $\tau$, we may assume that $\sigma_{s} \neq 0$ for any $s \in \mathbb{D}^{h(l, m, \beta)}$. In addition, $\sigma_{s} \rightarrow \sigma_{0} \otimes \sigma_{D}^{-\beta}$ for any $s \in \mathbb{D}^{h(l, m, \beta)}$ as $\tau \rightarrow 0$. Let $F_{s}$ be a smooth hypersurface defined by $\operatorname{div} \sigma_{s}=F_{s}-\beta D$. Since $\sigma_{0}$ contained in $\sigma_{s}$ is not multiplied by $\tau$, the variation of $\tau$ affects the choice of $F_{S}$ if $\tau \neq 0$.

By applying Theorem 4 , we obtain a complete Kähler metric $\omega_{F_{s}}$ with small scalar curvature for a meromorphic section $\sigma_{s} \otimes \sigma_{D}^{-m}$ of $K_{X}^{-l}$. In fact, for a smooth function on $X \backslash\left(D \cup F_{S}\right)$ defined by $b_{s}:=\log \left\|\sigma_{s}\right\|^{-2}$, we can obtain a Kähler metric $\sqrt{-1} \partial \bar{\partial} G_{v}^{\beta}\left(\beta b_{s}\right)$ on $X$. Directly, we have

$$
\sqrt{-1} \partial \bar{\partial} G_{v}^{\beta}\left(\beta b_{s}\right)=\left(\frac{1}{e^{-\beta b_{s}}+v}\right)^{1 / \beta}\left(\beta \sqrt{-1} \partial \bar{\partial} b_{s}+\frac{e^{-\beta b_{s}}}{e^{-\beta b_{s}}+v} \sqrt{-1} \partial b_{s} \wedge \bar{\partial} b_{s}\right) .
$$

This metric does not grow near $D$. When we glued plurisubharmonic functions in previous sections, we considered the Kähler potential $\kappa \Theta(t)+G_{v}^{\beta}\left(\beta b_{s}\right)$. In addition, 
$\lim _{b_{s} \rightarrow-\infty} \sqrt{-1} \partial \bar{\partial} G_{v}^{\beta}\left(\beta b_{s}\right)>-\infty$. So, we can construct a complete Kähler metric $\omega_{F_{s}}$ with small scalar curvature by using the regularized maximum $M_{\eta}$ in Lemma 20 to glue three plurisubharmonic functions $\Theta(t), \tilde{G}_{v}^{\beta}(b), t+\varphi+c$.

As has been pointed out previously in Remark $14,\left(X \backslash D, \omega_{F_{s}}\right)$ is not of asymptotically conical geometry for any $s \in \mathbb{D}^{h(l, m, \beta)}$. To solve this problem, consider an average metric $\bar{\omega}$ defined by

$$
\bar{\omega}=\bar{\omega}(c, v, \eta, \tau):=\int_{\mathbb{D}^{h(l, m, \beta)}} \omega_{F_{s}} d \mu(s)
$$

Here, $\mu$ is the Lebesgue probability measure on $\mathbb{D}^{h(l, m, \beta)}$ and $c, v, \eta$ are parameters in the definition of $\omega_{F}$ in Theorem 4. Recall that $\eta=\left(\eta_{1}, \eta_{2}, \eta_{3}\right)$ and $\eta_{1}, \eta_{2}=O(c), \eta_{3}=O(1)$. To prove that $(X \backslash D, \bar{\omega})$ is of asymptotically conical geometry, it is enough to prove the following lemma:

Lemma 32 For the Kähler metric $\bar{\omega}$ defined above, we have

$$
\bar{\omega}-\omega_{0}=O\left(\left\|\sigma_{D}\right\|^{2 \beta}\right)
$$

as $\sigma_{D} \rightarrow 0$.

Proof The region where $\left(X \backslash D, \omega_{F_{s}}\right)$ is not of asymptotically conical geometry is defined by

$$
\left|\Theta(t)-\tilde{G}_{v}^{\beta}\left(b_{s}\right)\right|<\eta_{1}+\eta_{2}
$$

(cf. Remark 14). For sufficiently large $b_{s}>0$, we have $v^{-1 / \beta} \beta b_{s} \approx G_{v}^{\beta}\left(\beta b_{s}\right)$. Here, $b_{s}:=$ $\log \left\|\sigma_{s}\right\|^{-2}$. From the following inequality

$$
v^{-1 / \beta} \beta b_{s} \approx G_{v}^{\beta}\left(\beta b_{s}\right)>(1-\kappa) \Theta(t)-\eta_{1}-\eta_{2}
$$

obtained by (63), we have

$$
\left\|\sigma_{s}\right\|^{2}<\exp \left(-\left(v^{1 / \beta} / \beta\right)\left((1-\kappa)\left\|\sigma_{D}\right\|^{-2 \hat{S}_{D} / n(n-1)}-\eta_{1}-\eta_{2}\right)\right) .
$$

Take a point $p \in X \backslash D$ near $D$. Assume that $\sigma_{\tilde{s}}(p)=0$ for $\tilde{s} \in \mathbb{D}^{h(l, m, \beta)}$. Then, an element $s \in \mathbb{D}^{h(l, m, \beta)}$ satisfying the inequality above has to satisfy

$$
\begin{aligned}
& \left\|\tau \sum_{i=1}^{h(l, m, \beta)}\left(s_{i}-\tilde{s}_{i}\right) \sigma_{i}(p) \otimes \sigma_{D}(p)^{-\beta}\right\|^{2} \\
& \quad<\exp \left(-\left(v^{1 / \beta} / \beta\right)\left((1-\kappa)\left\|\sigma_{D}\right\|^{-2 \hat{S}_{D} / n(n-1)}-\eta_{1}-\eta_{2}\right)\right) .
\end{aligned}
$$

By considering a suitable unitary transformation $u=\left(u_{i, j}\right) \in U(h(l, m, \beta))$, we can write as $\sum_{i=1}^{h(l, m, \beta)}\left(s_{i}-\tilde{s}_{i}\right) \sigma_{i}(p)=\left(\sum_{i, j=1}^{h(l, m, \beta)} u_{i, j}\left(s_{i}-\tilde{s}_{i}\right)\right) \tilde{\sigma}(p)$ for some holomorphic section $\tilde{\sigma} \in H^{0}\left(K_{X}^{-l} \otimes L_{X}^{m+\beta}\right)$ such that $\tilde{\sigma}(p) \neq 0$ and have

$$
\left|\sum_{i, j=1}^{h(l, m, \beta)} u_{i, j}\left(s_{i}-\tilde{s}_{i}\right)\right|^{2}<\frac{\exp \left(-\left(v^{1 / \beta} / \beta\right)\left((1-\kappa)\left\|\sigma_{D}\right\|^{-2 \hat{S}_{D} / n(n-1)}-\eta_{1}-\eta_{2}\right)\right)}{\tau^{2}\left\|\tilde{\sigma} \otimes \sigma_{D}^{-\beta}(p)\right\|^{2}} .
$$

Then, we have the following estimate

$$
\int \partial \Theta(t) \wedge \bar{\partial} \Theta(t) d \mu(s)=\exp \left(O\left(-\left\|\sigma_{D}\right\|^{-2 \hat{S}_{D} / n(n-1)}\right)\right) .
$$


Next, we consider the term

$$
\int \partial G_{v}^{\beta}\left(\beta b_{s}\right) \wedge \bar{\partial} G_{v}^{\beta}\left(\beta b_{s}\right) d \mu(s)
$$

which appears in $\bar{\omega}$. From the inequality (63), we have

$$
v^{-1 / \beta} \beta b_{s} \approx G_{v}^{\beta}\left(\beta b_{s}\right)<(1-\kappa) \Theta(t)+\eta_{1}+\eta_{2} .
$$

Thus, the following inequality

$$
\left\|\sigma_{s}\right\|^{-2}<\exp \left(\left(v^{1 / \beta} / \beta\right)\left((1-\kappa)\left\|\sigma_{D}\right\|^{-2 \hat{S}_{D} / n(n-1)}+\eta_{1}+\eta_{2}\right)\right)
$$

holds. Thus, we can estimate as follows

$$
\begin{aligned}
& \int \partial G_{v}^{\beta}\left(\beta b_{s}\right) \wedge \bar{\partial} G_{v}^{\beta}\left(\beta b_{s}\right) d \mu(s) \\
& \quad \leq \exp \left(2 v^{1 / \beta}\left(\eta_{1}+\eta_{2}\right) / \beta\right) / \tau^{2}\left\|\tilde{\sigma} \otimes \sigma_{D}^{-\beta}\right\|^{2} .
\end{aligned}
$$

By the definition of $\bar{\omega}$, we obtain

$$
\bar{\omega} \approx\left(1-\exp \left(-\left\|\sigma_{D}\right\|^{-2 \hat{S}_{D} / n(n-1)}\right)\right) \omega_{0}+O\left(\left\|\sigma_{D}\right\|^{2 \beta}\right)
$$

near $D$.

Proof of Theorem 5 Lemma 32 implies that the complete Kähler manifold $(X \backslash D, \bar{\omega})$ is of asymptotically conical geometry. Thus, we will prove that the scalar curvature can be made small arbitrarily. To show this, we take parameters $c, v, \tau$ and an integer $\beta$ so that

$$
v^{1 / \beta} c=k \log c, \quad \tau^{2}=v, \quad \beta>\delta
$$

for a sufficiently large $k \in \mathbb{N}$ specified later.

Firstly, from the construction of $\bar{\omega}$, weight norms of $S(\bar{\omega})$ away from $D \cup F_{0}$ can be made small arbitrarily by taking sufficiently small $\tau$. To show this, we study a function $f: \tau \rightarrow \bar{\omega}^{n} / \omega_{F_{0}}^{n}$. Note that this function is smooth and $f(0)=1$ and $\omega_{F_{0}}$ is Ricci-flat away from $D \cup F_{0}$. So, we have $S(\bar{\omega})=O(\tau)$ away from $D \cup F_{0}$.

Secondly, we study $S(\bar{\omega})$ near $F_{0}$ and away from $D$. We can write as

$$
\bar{\omega}=\int\left(\sqrt{-1} \partial \bar{\partial} M_{c, v, \eta}\right) d \mu,
$$

where

$$
\begin{aligned}
M_{c, v, \eta}= & \frac{\partial M_{c, v, \eta}}{\partial t_{2}}\left(\gamma_{v}^{\beta}+\kappa \omega_{0}\right)+\frac{\partial M_{c, v, \eta}}{\partial t_{3}} \sqrt{-1} \partial \bar{\partial}(t+\varphi) \\
& +\left[\partial \tilde{G}_{v}^{\beta}\left(b_{s}\right) \partial(t+\varphi)\right]\left[\frac{\partial^{2} M_{c, v, \eta}}{\partial t_{i} \partial t_{j}}\right]\left[\bar{\partial} \tilde{G}_{v}^{\beta}\left(b_{s}\right) \bar{\partial}(t+\varphi)\right]^{t} .
\end{aligned}
$$

On this region, we consider a sufficiently small neighborhood of $F_{0}$ by taking a sufficiently large parameter $c$. So, it is enough to consider the region defined by the following inequality

$$
G_{v}^{\beta}\left(b_{s}\right)+\kappa \Theta(t)-\eta_{2}>\max \left\{\Theta(t)+\eta_{1}, t+\varphi+c+\eta_{3}\right\} .
$$

In addition, since we are considering the region away from $D$, by taking a sufficiently large parameter $c$, the inequality above can be rewritten as follows:

$$
G_{v}^{\beta}\left(b_{s}\right)+\kappa \Theta(t)-(t+\varphi+c)>\eta_{2}+\eta_{3} .
$$


So, we have

$$
\left|\sum_{i}^{h(l, m, \beta)} s_{i}\right|^{2}<\exp \left(-\left(v^{1 / \beta} / \beta\right)\left(t+\varphi+c-\kappa \Theta(t)+\eta_{2}+\eta_{3}\right)\right) / \tau^{2}=O\left(c^{\beta-1}\right) .
$$

Recall Remark 7 and the relation of the parameters $c, v$ :

$$
c v^{1 / \beta}=k \log c \text {. }
$$

So, the inequality above (68) implies that

$$
\left\|\sigma_{F_{S}}\right\|^{2} \leq v^{k / \beta} .
$$

Thus, we don't have to consider the case that $S\left(\omega_{F}\right)=O(1)$ and we have

$$
\bar{\omega}=\int \gamma_{v}^{\beta} d \mu(s)+\kappa \omega_{0} \approx v^{-1 / \beta} \sqrt{-1} \partial \bar{\partial} b+\kappa \omega_{0} .
$$

by taking a sufficiently large $c$. Since the Ricci form of $\sqrt{-1} \partial \bar{\partial}\left(G_{v}^{\beta}\left(\beta b_{0}\right)+\kappa \Theta(t)\right)$ is bounded near $F_{0}$ and away from $D$, we can conclude that $S(\bar{\omega})=O\left(v^{1 / \beta}\right)$.

Thirdly, we study $S(\bar{\omega})$ near $D$. Write

$$
\bar{\omega}=\omega_{0}+\sqrt{-1} \partial \bar{\partial} \psi .
$$

By taking the trace with respect to the background metric $\omega_{0}$, we have

$$
\Delta_{\omega_{0}} \psi=\operatorname{tr}_{\omega_{0}} \bar{\omega}-n .
$$

To estimate

$$
S(\bar{\omega})=S\left(\omega_{0}+\sqrt{-1} \partial \bar{\partial} \psi\right),
$$

we study the right hand side in the equation above. Recall the construction of the complete Kähler metric $\omega_{F_{s}}$. The bounded region where plurisubharmonic functions $\Theta(t), t+\varphi+c$ are glued is defined by following inequalities:

$$
\begin{aligned}
\tilde{G}_{v}^{\beta}\left(b_{s}\right)+\eta_{2} & <\max \left\{\Theta(t)-\eta_{1},(t+\varphi+c)-\eta_{3}\right\}, \\
|\Theta(t)-(t+\varphi+c)| & <\eta_{1}+\eta_{3} .
\end{aligned}
$$

In addition, $\omega_{F_{s}}$ is written as

$$
\begin{aligned}
\omega_{F_{0}}= & \frac{\partial M_{c, v, \eta}}{\partial t_{1}} \omega_{0}+\frac{\partial M_{c, v, \eta}}{\partial t_{3}} \sqrt{-1} \partial \bar{\partial}(t+\varphi) \\
& +[\partial \Theta(t) \partial(t+\varphi)]\left[\frac{\partial^{2} M_{c, v, \eta}}{\partial t_{i} \partial t_{j}}\right][\bar{\partial} \Theta(t) \bar{\partial}(t+\varphi)]^{t} .
\end{aligned}
$$

Recall that $\eta_{1}+\eta_{3}=O(c)$. So, the inequality

$$
|\Theta(t)-(t+\varphi+c)|<\eta_{1}+\eta_{3}
$$

and Lemma 21 imply the following equivalence between complete Kähler metrics:

$$
\frac{\partial M_{c, v, \eta}}{\partial t_{1}} \omega_{0}<\omega_{F_{s}}<2 \omega_{0}
$$

on the region above. Next, we consider the region contained in the other region defined by

$$
v^{-1 / \beta} \beta b_{s} \approx G_{v}^{\beta}\left(\beta b_{s}\right)>(1-\kappa) \Theta(t)-\eta_{1}-\eta_{2} .
$$


In order to estimate $\operatorname{tr}_{\omega_{0}} \bar{\omega}-n$, it is enough to estimate the following terms

$$
c^{-1} \int \partial \Theta(t) \wedge \bar{\partial} \Theta(t) d \mu(s), \quad c^{-1} \int \partial G_{v}^{\beta}\left(\beta b_{s}\right) \wedge \bar{\partial} G_{v}^{\beta}\left(\beta b_{s}\right) d \mu(s) .
$$

Since $c \leq \Theta(t)$ on this region, the first term can be estimated as follows

$$
\begin{aligned}
& c^{-1}\left\|\sigma_{D}\right\|^{-4 \hat{S}_{D} / n(n-1)} \exp \left(-v^{1 / \beta}(1-\kappa)\left\|\sigma_{D}\right\|^{-2 \hat{S}_{D} / n(n-1)} / \beta\right) / \tau^{2}\left\|\tilde{\sigma} \otimes \sigma_{D}^{-\beta}\right\|^{2} \\
& =O\left(c^{1-(1-\kappa) k / \beta-n(n-1) \beta / \hat{S}_{D}+\beta}(\log c)^{\beta}\right)
\end{aligned}
$$

for parameters $\tau^{2}=v, c v^{1 / \beta}=k \log c$. From the estimate (66), the second term can be estimated as follows

$$
\begin{aligned}
c^{-1} & \int \partial G_{v}^{\beta}\left(\beta b_{s}\right) \wedge \bar{\partial} G_{v}^{\beta}\left(\beta b_{s}\right) d \mu(s) \\
& \leq \exp \left(2 v^{1 / \beta}\left(\eta_{1}+\eta_{2}\right) / \beta\right) / \tau^{2}\left\|\tilde{\sigma} \otimes \sigma_{D}^{-\beta}\right\|^{2} \\
& \leq O\left(c^{-1+2\left(a_{1}+a_{2}\right) k / \beta+\beta-n(n-1) \beta / \hat{S}_{D}}\right) .
\end{aligned}
$$

Recall the relation between parameters (62):

$$
1-\kappa+\kappa a_{1}-a_{2}=0 .
$$

Since the choice of $a_{i} \in(0,1)$ is independent of $\beta, k$, we can choose sufficiently small $a_{i}$ and $\kappa$ which is sufficiently close to 1 . Thus, we can make the following terms:

$$
\begin{aligned}
& 1-(1-\kappa) k / \beta-n(n-1) \beta / \hat{S}_{D}+\beta \\
& -1+2\left(a_{1}+a_{2}\right) k / \beta+\beta-n(n-1) \beta / \hat{S}_{D}
\end{aligned}
$$

negative by taking sufficiently large $\beta$ and $k$. Thus, we can estimate $\Delta_{\omega_{0}} \psi=\operatorname{tr}_{\omega_{0}} \bar{\omega}-n$ near $D$. From the equivalence (69), we obtain the following estimate near $F_{0}$ and away from $D$ :

$$
\operatorname{tr}_{\omega_{0}} \bar{\omega}-n=O\left(v^{-1 / \beta}\right)
$$

For any weight $\varepsilon \in(4,2 n)$, we have the following inequality

$$
\Delta_{\omega_{0}} \tilde{C} \rho^{-\varepsilon+2}<-C \rho^{-\varepsilon}<\Delta_{\omega_{0}} v^{1 / \beta} \psi<C \rho^{-\varepsilon}<-\Delta_{\omega_{0}} \tilde{C} \rho^{-\varepsilon+2}
$$

on $X \backslash D$ for some constants $C, \tilde{C}>0$ depending only on $\varepsilon$ and $n$. Here $\rho=\left\|\sigma_{D}\right\|^{-\hat{S}_{D} / n(n-1)}$ is the barrier function defined before (see [3]). Thus, the maximum principle tells us that there is the following $C_{\varepsilon-4}^{0}$-estimate of $\psi$ :

$$
\|\psi\|_{C_{\varepsilon-4}^{0}} \leq C v^{-1 / \beta}
$$

Recall the linearization of the scalar curvature operator

$$
S(\bar{\omega})=S\left(\omega_{0}\right)+L_{\omega_{0}}(\psi)+Q_{\omega_{0}}(\psi) .
$$

In addition, the term $Q_{\omega_{0}}(\psi)$ can be written as

$$
Q_{\omega_{0}}(\psi)=\left(L_{\omega_{0}+s \sqrt{-1} \partial \bar{\partial} \psi}-L_{\omega_{0}}\right)(\psi)
$$

for some $s \in[0,1]$ (see [17] or the proof of Lemma 16 in this article). Choose

$$
\varepsilon>\delta+2 \text {. }
$$

In this case, we can consider that $c \leq \Theta(t) \approx r^{2}$. 
Recall the interior Schauder estimate:

$$
\|\psi\|_{C_{\varepsilon-4}^{4, \alpha}} \leq C\left(\omega_{0}\right)\left(\left\|\operatorname{tr}_{\omega_{0}} \bar{\omega}-n\right\|_{C_{\varepsilon-2}^{2, \alpha}}+\|\psi\|_{C_{\varepsilon-4}^{0}}\right) .
$$

Here, $C\left(\omega_{0}\right)$ is a positive constant depending only on $\omega_{0}$. The previous estimate (70) implies that $\|\psi\|_{C_{\varepsilon-4}^{4, \alpha}}=O\left(v^{-1 / \beta}\right)$. Then, the equality (71) implies that the norm of scalar curvature of weight $\delta$ is estimated from above by $c^{(\delta-\varepsilon) / 2+1}(\log c)^{-1}$. In these settings of parameters, we show finally that the scalar curvature on the region defined by

$$
t+\varphi+c-\eta_{3}>\max \left\{\Theta(t)+\eta_{1}, \tilde{G}_{v}^{\beta}\left(b_{s}\right)+\eta_{2}\right\}
$$

can be estimated in the sense of weighted norms. It follows from the first discussion that $S(\bar{\omega})=O(\tau)$ on the region above. Then, we have

$$
S(\bar{\omega})=O(\tau)=O\left(c^{-\beta / 2}(k \log c)^{\beta / 2}\right) .
$$

Recall that $\Theta(t) \approx r^{2} \approx c$ on this region. So, we can estimate the $C^{k, \alpha}$-norm of the function $S(\bar{\omega})\left(r^{2}+1\right)^{\delta / 2} \approx S(\bar{\omega}) c^{\delta / 2}$ in the definition of the weighted norm (Definition 5) on this region. More precisely, the choice of $\beta$ :

$$
\beta>\delta
$$

implies that we can estimate the $\delta$-weighted norm of the scalar curvature $S(\bar{\omega})$ on the region above. Therefore, from the discussion above, we can conclude that the weight norm of $S\left(\omega_{c, v, \eta}\right)$ can be made small arbitrarily by taking a sufficiently large parameter $c$ (equivalently, sufficiently small parameters $v, \tau)$. In addition, from the linearization of scalar curvatures, the scalar curvature $S(\bar{\omega})$ decays just like $S\left(\omega_{0}\right)$. Thus, we finish the proof of Theorem 5 .

Remark 15 If $\theta_{D}$ is cscK, Theorem 1 implies that we have

$$
S(\bar{\omega})=O\left(\left\|\sigma_{D}\right\|^{2+2 \hat{S}_{D} / n(n-1)}\right)=O\left(r^{-2-2 n(n-1) / \hat{S}_{D}}\right)
$$

near $D$.

Remark 16 Recall that we choose a parameter $v>0$ so that the inequality $\left(\left\|\sigma_{F}\right\|^{2 \beta}+v\right)^{2 / \beta}<$ $\left\|\sigma_{F}\right\|^{4 a m / l}$ holds on the region defined by

$$
\begin{aligned}
\Theta(t)+\eta_{1} & <\max \left\{\tilde{G}_{v}^{\beta}(b)-\eta_{2},(t+\varphi+c)-\eta_{3}\right\}, \\
\left|\tilde{G}_{v}^{\beta}(b)-(t+\varphi+c)\right| & <\eta_{2}+\eta_{3} .
\end{aligned}
$$

Note that $G_{v}^{\beta}(\beta b) \approx \beta v^{-1 / \beta} b$ for sufficiently large $b>0$. The choice of parameters $c v^{1 / \beta}=$ $k \log c$ in the previous theorem implies that we have $\left\|\sigma_{F}\right\|^{-2 \beta} \approx v^{k}$. Therefore, we can choose a suitable parameter $v>0$ so that $\left(\left\|\sigma_{F}\right\|^{2 \beta}+v\right)^{2 / \beta}<\left\|\sigma_{F}\right\|^{4 a m / l}$ without contradiction.

\section{Proof of Theorem 6}

After this, all weighted Banach spaces $C_{\delta}^{k, \alpha}=C_{\delta}^{k, \alpha}(X \backslash D)$ are defined by the fixed Kähler metric $\omega_{0}$. In Theorem 6, we assume that

$$
0<3 \hat{S}_{D}<n(n-1)
$$

we choose a weight $\delta$ so that

$$
8<\delta<\min \left\{2 n, 2+2 n(n-1) / \hat{S}_{D}\right\}
$$


and a function

$$
\phi \mathscr{D}_{\bar{\omega}}^{*} \mathscr{D}_{\bar{\omega}} \phi
$$

is integrable for $\phi \in C_{\delta-4}^{4, \alpha}$ with respect to the volume form $\bar{\omega}^{n}$. In addition, we may assume that the integer $a(n)$ in Theorem 4 satisfies

$$
12 / a(n)<\delta-8<\min \left\{2 n-8,2 n(n-1) / \hat{S}_{D}-6\right\} .
$$

\subsection{Condition 1 and Condition 2}

In this subsection, we show that Condition 1 and Condition 2 in Introduction of this article hold with respect to the complete Kähler metric $\bar{\omega}$ obtained in the previous section.

In order to find the constant $\hat{K}$ in Condition 1, we use the resonance theorem (see [21, p. 69]).

Theorem 13 (the resonance theorem) Let $\left\{T_{a} \mid a \in A\right\}$ be a family of bounded linear operators defined on a Banach space $\mathscr{X}$ into a normed linear space $\mathscr{Y}$. Then, the boundedness of $\left\{\left\|T_{a} x\right\| \quad \mid a \in A\right\}$ for each $x \in \mathscr{X}$ implies the boundedness of $\left\{\left\|T_{a}\right\| \quad \mid a \in A\right\}$.

Then, we obtain the following theorem which is the core of this article:

Theorem 14 Take parameters $c, v, \tau$ so that $v^{1 / \beta} c=k \log c, \tau^{2}=v$. Assume that there is no nonzero holomorphic vector field on $X$ which vanishes on $D$. Then, there exists an uniform constant $K>0$ such that

$$
\left\|\mathscr{D}_{\bar{\omega}}^{*} \mathscr{D}_{\bar{\omega}} \phi\right\|_{C_{\delta}^{0, \alpha}} \geq K\|\phi\|_{C_{\delta-4}^{4, \alpha}}
$$

for any $c, v, \eta, \tau$ and $\phi \in C_{\delta-4}^{4, \alpha}$.

Proof We prove this theorem by using Theorem 13. So, for a fixed function $\phi \in C_{\delta-4}^{4, \alpha}$, it is enough to show that the quantity

$$
\frac{\|\phi\|_{C_{\delta-4}^{4, \alpha}}}{\left\|\mathscr{D}_{\bar{\omega}}^{*} \mathscr{D}_{\bar{\omega}} \phi\right\|_{C_{\delta}^{0, \alpha}}}
$$

has an upper bound depending only on $\phi$. We prove this by contradiction. Assume that there exists a sequence $(\tau, v, c) \rightarrow(0,0, \infty)$ such that $\left\|\mathscr{D}_{\bar{\omega}}^{*} \mathscr{D}_{\bar{\omega}} \phi\right\|_{C_{\delta}^{0, \alpha}} \rightarrow 0$ for some $\phi \in C_{\delta-4}^{4, \alpha}$ with $\|\phi\|_{C_{\delta-4}^{4, \alpha}}=1$. By integration by parts, we have

$$
\int_{X \backslash D} \phi \mathscr{D}_{\bar{\omega}}^{*} \mathscr{D} \bar{\omega} \phi \bar{\omega}^{n}=\int_{X \backslash D}|\mathscr{D} \bar{\omega} \phi|^{2} \bar{\omega}^{n}
$$

Recall that $\mathscr{D}_{\bar{\omega}} \rightarrow \mathscr{D}_{\sqrt{-1} \partial \bar{\partial}(t+\varphi)}$ as $(\tau, v, c) \rightarrow(0,0 . \infty)$. We show that

$$
\int_{X \backslash D} \phi \mathscr{D}_{\bar{\omega}}^{*} \mathscr{D}_{\bar{\omega}} \phi \bar{\omega}^{n} \rightarrow 0
$$

as $(\tau, v, c) \rightarrow(0,0, \infty)$. To see this, we study the volume of the subset $\cup_{s \in \mathbb{D}^{h(l, m+1)}} F_{s}$. For $p \in X \backslash D$ close to $F_{0}$, we can find $s \in \mathbb{D}^{h(l, m+1)}$ such that $\sigma_{s}(p)=0$. So, we have

$$
\left\|\sigma_{0}(p)\right\| \leq\left\|\sigma_{i}(p)\right\|+\tau\left\|\sum_{i} s_{i} \sigma_{i}(p)\right\| \leq C \tau .
$$


On the other hand, $\bar{\omega}<v^{-1 / \beta} \sqrt{-1} \partial \bar{\partial} b_{0}$ near $F_{0}$. Thus, we have

$$
\int_{\cup_{s \in \mathbb{D}^{h}(l, m, \beta)} F_{s}} \bar{\omega}^{n}<O\left(\tau^{2} v^{-n / \beta}\right)=O\left(v^{1-n / \beta}\right) .
$$

It follows from the choice of $v>0$ in this theorem that the desired convergence above holds as $(\tau, v, c) \rightarrow(0,0, \infty)$ by taking sufficiently large $\beta$. Then, we obtain a holomorphic vector field

$$
\nabla^{1,0} \phi=g^{i, \bar{j}} \frac{\partial \phi}{\partial \bar{z}^{j}} \frac{\partial}{\partial z^{i}}
$$

on $X \backslash\left(D \cup F_{0}\right)$. Here, we write $\sqrt{-1} \partial \bar{\partial}(t+\varphi)=\sqrt{-1} g_{i, \bar{j}} d z^{i} \wedge d \bar{z}^{j}$. It follows from the definitions of $\phi$ and the $C^{2}$-estimate of $g^{i, \bar{j}}$ (Theorem 12) that $\nabla^{1,0} \phi$ can be extended to $X$. The decay condition of $\phi$ and the assumption of holomorphic vector fields on $X$ imply that $\phi=0$. This is contradiction and the resonance theorem (Theorem 13) implies that the inverse operator $\mathscr{D}_{\bar{\omega}}^{*} \mathscr{D}_{\bar{\omega}}^{-1}$ has an uniform bound.

Recall the following relation

$$
L_{\bar{\omega}}=-\mathscr{D}_{\bar{\omega}}^{*} \mathscr{D}_{\bar{\omega}}+\left(\nabla^{1,0} *, \nabla^{0,1} S(\bar{\omega})\right)_{\bar{\omega}} .
$$

Thus, Theorems 5 and 14 imply that Condition 1 holds with respect to $\bar{\omega}$.

Theorem 15 Take parameters so that $v^{1 / \beta} c=k \log c$ and $\tau^{2}=v$. Assume that $\theta_{D}$ is cscK and $\mathscr{D}_{\bar{\omega}}^{*} \mathscr{D}_{\bar{\omega}}: C_{\delta-4}^{4, \alpha} \rightarrow C_{\delta}^{0, \alpha}$ is isomorphic. Then, we can make the norm of the linear operator $\left(\nabla^{1,0} *, \nabla^{0,1} S(\bar{\omega})\right)_{\bar{\omega}}=L_{\bar{\omega}}+\mathscr{D}_{\bar{\omega}}^{*} \mathscr{D}_{\bar{\omega}}$ small arbitrarily so that $L_{\bar{\omega}}: C_{\delta-4}^{4, \alpha} \rightarrow C_{\delta}^{0, \alpha}$ is isomorphic. Moreover, we can find a constant $\hat{K}>0$ such that

$$
\left\|L_{\bar{\omega}} \phi\right\|_{C_{\delta}^{0, \alpha}} \geq \hat{K}\|\phi\|_{C_{\delta-4}^{4, \alpha}}
$$

for any $c, v, \tau, \phi \in C_{\delta-4}^{4, \alpha}$.

We need the following modified lemma:

Lemma 33 Assume that $n \geq 5$ and

$$
3 \hat{S}_{D}<n(n-1) .
$$

Then, for $\delta>8$, there exists $c_{0}>0$ independent of $\bar{\omega}$ such that if $\|\phi\|_{C_{\delta-4}^{4, \alpha}(X \backslash D)} \leq c_{0}$, we have

$$
\left\|L_{\bar{\omega}_{\phi}}-L_{\bar{\omega}}\right\|_{C_{\delta-4}^{4, \alpha} \rightarrow C_{\delta}^{0, \alpha}} \leq \hat{K} / 2
$$

and $\bar{\omega}_{\phi}=\bar{\omega}+\sqrt{-1} \partial \bar{\partial} \phi$ is positive.

Proof The proof of this lemma is similar to the proof of Lemma 15. For $\psi \in C_{\delta-4}^{4, \alpha}$, the following inequality holds:

$$
\begin{aligned}
& \left\|\left(r^{2}+1\right)^{\delta / 2}\left(g_{\phi}^{i, \bar{j}} g_{\phi}^{k, \bar{l}}-g^{i, \bar{j}} g^{k, \bar{l}}\right) \psi_{i, \bar{j}, k, \bar{l}}\right\|_{C^{0, \alpha}} \\
& \leq\left\|\left(r^{2}+1\right)^{4 / 2}\left(g_{\phi}^{i, \bar{j}} g_{\phi}^{k, \bar{l}}-g^{i, \bar{j}} g^{k, \bar{l}}\right)\right\|_{C^{0, \alpha}}\|\psi\|_{C_{\delta-4}^{4, \alpha}(X \backslash D)} \\
& \quad=\left\|\left(r^{2}+1\right)^{4 / 2}\left(g_{\phi}^{i, \bar{j}}\left(g_{\phi}^{k, \bar{l}}-g^{k, \bar{l}}\right)+\left(g_{\phi}^{i, \bar{j}}-g^{i, \bar{j}}\right) g^{k, \bar{l}}\right)\right\|_{C^{0, \alpha}}\|\psi\|_{C_{\delta-4}^{4, \alpha}(X \backslash D)}
\end{aligned}
$$


In addition, we have the following equation:

$$
g_{\phi}^{-1}-g^{-1}=g_{\phi}^{-1}\left(g-g_{\phi}\right) g^{-1}
$$

for $\phi \in C_{\delta-4}^{4, \alpha}$ such that $\bar{\omega}_{\phi}=\bar{\omega}+\sqrt{-1} \partial \bar{\partial} \phi$ is positive.

It is enough to study the region where $M_{c, v, \eta}=t+\varphi+c$. The $C^{2}$-estimate of the degenerate complex Monge-Ampère equation (Theorem 12) tells us that

$$
g^{i, \bar{j}}=O\left(\left\|\sigma_{D}\right\|^{-2 m / l}\right)=O\left(r^{2 m / l \times n(n-1) / \hat{S}_{D}}\right) .
$$

Since we have already known the explicit $C^{2, \varepsilon}$-estimate of the solution of the degenerate complex Monge-Ampère equation from Proposition 11, we can estimate the $C^{0, \alpha}$-norm of coefficients $g^{i, \bar{j}}, g_{\phi}^{i, \bar{j}}$. The hypothesis

$$
\frac{a(n) m}{2 l}<\frac{\hat{S}_{D}}{n(n-1)}
$$

implies that $4+3 \times 2 m / l \times n(n-1) / \hat{S}_{D}-(\delta-4)<8+12 / a(n)-\delta<0$. So, the Eq. (73) and the estimate (74) implies that the term

$$
\left\|\left(r^{2}+1\right)^{4 / 2}\left(g_{\phi}^{i, \bar{j}}\left(g_{\phi}^{k, \bar{l}}-g^{k, \bar{l}}\right)+\left(g_{\phi}^{i, \bar{j}}-g^{i, \bar{j}}\right) g^{k, \bar{l}}\right)\right\|_{C^{0, \alpha}}
$$

is estimated form above by $2 c_{0}$. By taking a sufficiently small $c_{0}$, we can make the operator norm of $L_{\bar{\omega}_{\phi}}-L_{\bar{\omega}}$ small arbitrarily. Thus, we have the desired result.

Remark 17 The reason why we replace the hypothesis for weights of Banach spaces in the above lemma comes from the $C^{2}$-estimate of the solution of the degenerate complex MongeAmpère equation due to Păun [15] (see Theorem 12 in this article). From this, the positivity of $\bar{\omega}_{\phi}$ holds. On the other hand, we need to assume that $\delta-4>4$ to control the factor $\left(r^{2}+1\right)^{4 / 2}$. So, the choice of a weight $\delta$ implies that we need to assume that the dimension $n$ is greater than 4 and $\hat{S}_{D} / n(n-1)$ is smaller than $1 / 3$. In addition, since we need to choose $\varepsilon>\delta+2$ in the proof of Theorem 5, we need to assume that $n>5$.

Constants $\hat{K}$ and $c_{0}$ which appear in Theorem 15 and Lemma 33 respectively, are uniform for parameters $c, v, \tau$. Therefore, Theorem 5 implies that Condition 2 holds with respect to $\bar{\omega}$.

Theorem 16 For the complete Kähler metric $\bar{\omega}$ above, the inequality

$$
\|S(\bar{\omega})\|_{C_{\delta}^{0, \alpha}} \leq c_{0} \hat{K} / 2
$$

holds by taking suitable parameters $v, c, \tau$.

\subsection{The fixed point theorem}

Finally, we show that the existence of a complete scalar-flat Kähler metric on $X \backslash D$. Recall that for the expansion of the scalar curvature

$$
S(\bar{\omega}+\sqrt{-1} \partial \bar{\partial} \phi)=S(\bar{\omega})+L_{\bar{\omega}}(\phi)+Q_{\bar{\omega}}(\phi),
$$

we consider the following operator

$$
\mathscr{N}(\phi):=-L_{\bar{\omega}}^{-1}\left(S(\bar{\omega})+Q_{\bar{\omega}}(\phi)\right) \in C_{\delta-4}^{4, \alpha}
$$


for $\phi \in C_{\delta-4}^{4, \alpha}$ by following Arezzo-Pacard [1,2] (see also [17]). Lemma 33 implies that $\mathscr{N}$ is the contraction map on the neighborhood of the origin of $C_{\delta-4}^{4, \alpha}$ for a suitable weight $\delta$. In Proposition 9, we assume that Conditions 1 and 2 hold. Namely, we assume that there exists a complete Kähler metric $\omega_{0}$ whose scalar curvature is sufficiently small so that the operator $L_{\omega_{0}}$ has the uniformly bounded inverse. As we have seen, by constructing the Kähler metric $\bar{\omega}$, Theorem 15 and Theorem 16 imply that we don't have to assume that Conditions 1 and 2 hold. The following Proposition implies the existence of a complete scalar-flat Kähler metric.

Proposition 14 Set

$$
\mathscr{U}:=\left\{\phi \in C_{\delta-4}^{4, \alpha}:\|\phi\|_{C_{\delta-4}^{4, \alpha}} \leq c_{0}\right\} .
$$

If the assumption in Theorem 6 holds, the operator $\mathscr{N}$ is a contraction on $\mathscr{U}$ and $\mathscr{N}(\mathscr{U}) \subset \mathscr{U}$ by taking suitable parameters $c, v, \tau$.

Proof Immediately, we have

$$
\|\mathscr{N}(\phi)\|_{C_{\delta-4}^{4, \alpha}} \leq\|\mathscr{N}(\phi)-\mathscr{N}(0)\|_{C_{\delta-4}^{4, \alpha}}+\|\mathscr{N}(0)\|_{C_{\delta-4}^{4, \alpha}} .
$$

From Lemma 33 and the condition $\|\phi\|_{C_{\delta-4}^{4, \alpha}} \leq c_{0}$, the same argument in the proof of Lemma 16 implies that we obtain the following estimate:

$$
\begin{aligned}
\|\mathscr{N}(\phi)-\mathscr{N}(0)\|_{C_{\delta-4}^{4, \alpha}} & \leq\left\|-L_{\bar{\omega}}^{-1}\left(Q_{\bar{\omega}}(\phi)\right)\right\|_{C_{\delta-4}^{4, \alpha}} \\
& \leq\left.\hat{K}^{-1}\left\|L_{\bar{\omega}+s \sqrt{-1} \partial \bar{\partial} \phi}-L_{\bar{\omega}}\right\|_{C_{\delta-4}^{4, \alpha} \rightarrow C_{\delta}^{0, \alpha}}\|\phi\|\right|_{C_{\delta-4}^{4, \alpha}}
\end{aligned}
$$

for some $s \in[0,1]$. Lemma 33 implies that we have

$$
\|\mathscr{N}(\phi)-\mathscr{N}(0)\|_{C_{\delta-4}^{4, \alpha}} \leq \frac{1}{2} c_{0} .
$$

Theorems 15 and 16 implies that we have

$$
\|\mathscr{N}(0)\|_{C_{\delta-4}^{4, \alpha}}=\left\|L_{\bar{\omega}}^{-1}(S(\bar{\omega}))\right\|_{C_{\delta-4}^{4, \alpha}} \leq \hat{K}^{-1}\|S(\bar{\omega})\|_{C_{\delta}^{0, \alpha}} \leq \frac{1}{2} c_{0} .
$$

Thus, $\mathscr{N}(\phi) \in \mathscr{U}$.

Proof of Theorem 6 From the discussion above, there exists a unique $\phi_{\infty}:=\lim _{i \rightarrow \infty} \mathscr{N}^{i}(\phi)$ for any $\phi \in \mathscr{U} \subset C_{\delta-4}^{4, \alpha}$ satisfying $\phi_{\infty}=\mathscr{N}\left(\phi_{\infty}\right)$ under the hypothesis in Theorem 6 . Therefore, $\bar{\omega}+\sqrt{-1} \partial \bar{\partial} \phi_{\infty}$ is a complete scalar-flat Kähler metric on $X \backslash D$.

Acknowledgements The author would like to thank Professor Ryoichi Kobayashi who first brought the problem in this article to his attention, for many helpful comments. In particular, the author learned the idea of using the complex Monge-Ampère equation to scalar curvatures from him.

Open Access This article is licensed under a Creative Commons Attribution 4.0 International License, which permits use, sharing, adaptation, distribution and reproduction in any medium or format, as long as you give appropriate credit to the original author(s) and the source, provide a link to the Creative Commons licence, and indicate if changes were made. The images or other third party material in this article are included in the article's Creative Commons licence, unless indicated otherwise in a credit line to the material. If material is not included in the article's Creative Commons licence and your intended use is not permitted by statutory regulation or exceeds the permitted use, you will need to obtain permission directly from the copyright holder. To view a copy of this licence, visit http://creativecommons.org/licenses/by/4.0/. 


\section{References}

1. Arezzo, C., Pacard, F.: Blowing up and desingularizing constant scalar curvature Kähler manifolds. Acta Math. 196(2), 179-228 (2006)

2. Arezzo, C., Pacard, F.: Blowing up Kähler manifolds with constant scalar curvature II. Ann. Math. (2) 170(2), 685-738 (2009)

3. Bando, S., Kobayashi, R.: Ricci-flat Kähler metrics on affine algebraic manifolds. II. Math. Ann. 287, 175-180 (1990)

4. Błocki, Z.: On the regularity of the complex Monge-Ampère operator, Complex Geometric Analysis n Pohang (1997). Contemp. Math., vol. 222, Amer. Math. Soc., Providence, pp. 181-189 (1999)

5. Calabi, E.: Métriques Kähleriennes et fibrés holomorphes. Ann. Sci. École Norm. Sup. (4) 12(2), 269-294 (1979)

6. Demailly, J.-P.: Complex analytic and differential geometry, online book available at http://www-fourier. ujf-grenoble.fr/ demailly/manuscripts/agbook.pdf

7. Di Nezza, E., Lu, H.C.: Complex Monge-Ampère equations on quasi-projective varieties. J. Reine Angew. Math. (2014). https://doi.org/10.1515/crelle-2014-0090

8. Gilbarg, D., Trudinger, N.S.: Elliptic Partial Differential Equations of Second Order, 2nd edn., Grundlehren Math. Wiss., vol. 224. Springer, Berlin (1983)

9. Gilkey, P.B.: Invariance Theory, the Heat Equation, and the Atiyah-Singer Index Theorem, 2nd edn. CRC Press, Boca Raton (1995)

10. Guedj, V., Zeriahi, A.: Degenerate Complex Monge-Ampère Equations, EMS Tracts Math (2017)

11. Hein, H.-J.: Weighted Sobolev inequalities under lower Ricci curvature bounds. Proc. Am. Math. Soc. 139(8), 2943-2955 (2011)

12. Hwang, A.D., Singer, M.A.: A momentum construction for circle-invariant Kähler metrics. Trans. Am. Math. Soc. 354(6), 2285-2325 (2002)

13. Kołodziej, S.: The complex Monge-Ampère equation. Acta Math. 180, 69-117 (1998)

14. LeBrun, C.: Counter-examples to the generalized positive action conjecture. Commun. Math. Phys. 118(4), 591-596 (1988)

15. Păun, M.: Regularity properties of the degenerate Monge-Ampère equations on compact Kähler manifolds. Chin. Ann. Math. Ser. B. 29, 623-630 (2008)

16. Simanca, S.R.: Kähler metrics of constant scalar curvature on bundles over $\mathbb{C P}^{n-1}$. Math. Ann. 291, 239-246 (1991)

17. Székelyhidi, G.: An Introduction to Extremal Kähler Metrics, Graduate Studies in Mathematics, vol. 152. Amer. Math. Soc, Providence (2014)

18. Tian, G., Yau, S.T.: Complete Kähler manifolds with zero Ricci curvature. I. J. Am. Math. Soc. 3, 579-610 (1990)

19. Tian, G., Yau, S.T.: Complete Kähler manifolds with zero Ricci curvature. II. Invent. Math. 106(1), 27-60 (1991)

20. Yau, S.T.: On the Ricci curvature of a compact Kähler manifold and the complex Monge-Ampère equations, I. Commun. Pure Appl. Math. 31, 339-411 (1978)

21. Yosida, K.: Functional Analysis, 2nd edn. Springer, Berlin (1968)

22. Zhan, X.: Matrix Theory, Graduate Studies in Mathematics, vol. 147. Amer. Math. Soc., Providence (2013)

Publisher's Note Springer Nature remains neutral with regard to jurisdictional claims in published maps and institutional affiliations. 